1

\title{
Title Differential requirements of cyclase associated protein (CAP) for actin turnover during the lytic cycle of Toxoplasma gondii
}

\author{
Alex Hunt ${ }^{1}$, Jeanette Wagener ${ }^{1}$, Robyn Kent ${ }^{2}$, Romain Carmeille ${ }^{3}$, Matt Russell ${ }^{4}$, Christopher \\ Peddie $^{4}$, Lucy Collinson ${ }^{4}$, Aoife Heaslip ${ }^{3}$, Gary E. Ward ${ }^{2} \&$ Moritz Treeck ${ }^{1 *}$ \\ ${ }^{1}$ Signalling in Apicomplexan Parasites Laboratory, The Francis Crick Institute, United Kingdom \\ ${ }^{2}$ Department of Microbiology and Molecular Genetics, University of Vermont Larner College of \\ Medicine, Burlington, Vermont, United States \\ ${ }^{3}$ Department of Molecular and Cell Biology, University of Connecticut, Connecticut, United \\ States \\ ${ }^{4}$ Electron Microscopy Science Technology Platform, The Francis Crick Institute, United \\ Kingdom
}

*For correspondence: moritz.treeck@crick.ac.uk

\begin{abstract}
Toxoplasma gondii contains a limited subset of actin binding proteins. Here we show that ablation of the putative actin regulator cyclase-associated protein (TgCAP) leads to significant defects in some but not all actin dependent processes, including a defect in cell-cell communication, but surprisingly not synchronicity of division. Two CAP isoforms originate from alternative translational start sites and are beneficial for parasite fitness while a single isoform is sufficient for virulence in mice. Examination of the mutant parasites by $3 \mathrm{D}$ electron microscopy reveals that loss of CAP results in a defect to form a normal residual body, but all parasites remain connected within the vacuole. This dissociates synchronicity of division and parasite rosetting and reveals that establishment and maintenance of the residual body may be more complex than previously thought. These results highlight the different spatial requirements for actin turnover in Toxoplasma, controlled by a reduced subset of actin binding proteins.
\end{abstract}

\section{Introduction}

Toxoplasma gondii is an obligate intracellular parasite, belonging to the Apicomplexa phylum. The Apicomplexa, which also include Plasmodium and Cryptosporidium species, pose a significant global public health burden. Toxoplasma, specifically, is one of the most prevalent human pathogens, chronically infecting $\sim 30 \%$ of the world's population (Swapna \& Parkinson, 2017). While most infections are asymptomatic, in congenitally infected and immunocompromised patients, disease outcomes are often severe and potentially fatal (Halonen \& Weiss, 2013). During acute infection of the host, the asexual tachyzoite stage of Toxoplasma gondii undergoes cycles of active invasion, replication and egress from host cells. This lytic cycle leads to rapid proliferation and dissemination of the parasite throughout the host (Black \& Boothroyd, 2000). To facilitate these processes, Toxoplasma utilises a unique form of locomotion, called gliding motility, which relies on actin and an unconventional myosin motor (Frénal, Dubremetz, et al., 2017). This motor allows the parasite to actively invade host cells, where it forms a protective parasitophorous vacuole (PV). PV structural integrity and stability is sustained through the parasite's release of dense granule proteins from secretory vesicles (Heaslip et al., 2016). Additionally, several dense granule proteins are transported into the host cell where they co-opt or interfere with host cell functions (Hakimi et al., 2017). Within the PV, Toxoplasma begins a unique form of cell division called endodyogeny (Sheffield \& Melton, 1968). Here, two daughter cells are synchronously assembled within the mother cell before daughter cell budding (Delbac et al., 2001). This initiates at the apical pole of the mother cell and once complete, the daughter cells bud from the mother cell but remain attached at their basal pole to the residual body: a membranous compartment containing maternal remnants of organelles and cytoskeletal elements (Muñiz-Hernández et al., 2011). During cell division, organelles such as mitochondria and the apicoplast, a unique and essential organelle 
which contributes to isoprenoid synthesis (Vaishnava \& Striepen, 2006), also divide and are distributed between the two daughter parasites. Following multiple rounds of cell division, parasites organise into a rosette-like pattern around the residual body. Formation of the characteristic rosette pattern requires actin, several myosins, and other actin-binding proteins (Frénal, Jacot, et al., 2017; Haase et al., 2015; Jacot et al., 2013; Periz et al., 2017; Tosetti et al., 2019). It has been hypothesised that the inter-parasite connections via the residual body are not only important for parasite organisation, but also play a key role in cell-cell communication. Such communication was measured by the transfer of reporter proteins between parasites and is believed to ensure the synchronous division of parasites within a vacuole (Frénal, Jacot, et al., 2017). The parasites continue to replicate until host cell lysis and parasite egress. Following egress, parasites migrate to and invade new host cells and the lytic cycle repeats, leading to tissue destruction (Black \& Boothroyd, 2000). Actin plays an essential role in the parasite's lytic cycle through function of the actin cytoskeleton and actomyosin motor complex. Despite this crucial role in apicomplexan biology, there has been difficulty visualising actin filaments in apicomplexan species (Bannister \& Mitchell, 1995; Sahoo et al., 2006; Shaw \& Tilney, 1999) and it has been suggested that as much as $98 \%$ of parasite actin is monomeric (G-actin) and not incorporated into filaments (F-actin) (Dobrowolski et al., 1997). This, along with structural differences found in actin of Apicomplexa (Pospich et al., 2017), led to the hypothesis that Toxoplasma F-actin has reduced stability, forming abnormally short filaments that are rapidly recycled to maintain essential cellular function (Pospich et al., 2017; Skillman et al., 2011). However, recent development of the actin-chromobody has allowed for the visualisation of long F-actin structures both in the parasite and extensive networks within the PV (Periz et al., 2017). Taken together, along with biochemical evidence, apicomplexan actin appears to be different to actin from other organisms (Frénal, Dubremetz, et al., 2017).

Actin turnover is regulated by actin binding proteins, of which Toxoplasma possesses a reduced repertoire, including ADF/cofilin, profilin, coronin and cyclase-associated protein (CAP) (Baum et al., 2006). Functional studies have shown ADF/cofilin and profilin to be essential for Toxoplasma progression through the lytic cycle, while coronin depletion had a modest impact on parasite invasion and egress (Mehta \& Sibley, 2011; Plattner et al., 2008; Salamun et al., 2014). Apart from its localisation, the function of CAP in Toxoplasma has not been investigated. In the majority of eukaryotes, CAP is a highly conserved multidomain protein that regulates actin filament dynamics via two distinct mechanisms (Ono, 2013). CAP can bind and sequester G-actin using its CAP and X-linked retinitis pigmentosa 2 protein (CARP) domain and can regulate actin filament disassembly by promoting ADF/cofilin mediated severing using its helical folded domain (HFD). Through regulation of actin dynamics, by interacting with actin and other actin binding proteins, it has been shown that mouse CAP1 plays important roles in cell morphology, migration and endocytosis (Bertling et al., 2004). However, species belonging to the Apicomplexa phylum possess a truncated form of CAP, retaining only the conserved C-terminal G-actin-binding CARP domain (Hliscs et al., 2010). This conserved $\beta$-sheet domain has been shown to interact directly with monomeric actin, providing either sequestration or nucleotide exchange of G-actin in a concentration-dependent manner (Hliscs et al., 2010; Makkonen et al., 2013; Mattila et al., 2004). As such, apicomplexan CAP is hypothesised to regulate actin turnover solely through interaction with monomeric actin. Biochemical analysis of Cryptosporidium parvum CAP identified the formation of a dimer and G-actin sequestering activity, while Plasmodium falciparum CAP was shown to facilitate nucleotide exchange, loading ADP-actin monomers with ATP (Hliscs et al., 2010; Makkonen et al., 2013). A Plasmodium berghei CAP KO demonstrated that while PbCAP is dispensable for asexual blood stages in vivo, there is a complete defect in oocyst development in the insect vector which was overcome through complementation with C. parvum CAP (Hliscs et al., 2010). A PbCAP overexpression study revealed no defect in ookinete motility or oocyst development, however sporozoites displayed impaired gliding motility, invasion and salivary gland colonisation (Sato et al., 2016). Taken together, these results suggest that apicomplexan CAP may function as a dimer with the ability to interact with G-actin monomers to sequester them and/or facilitate their nucleotide exchange. While CAP has yet to be functionally 
characterised in Toxoplasma, Lorestani et al reported that CAP localises to the apex of intracellular parasites, a hub for events leading to egress and motility (Graindorge et al., 2016; Lorestani et al., 2012; Tosetti et al., 2019). Intriguingly, following host cell lysis, relocalisation of CAP to the parasite cytosol was observed (Lorestani et al., 2012). Furthermore, we have previously identified differential phosphorylation of TgCAP in parasites with a delayed egress phenotype (Treeck et al., 2014). The correlation between TgCAP redistribution and phosphorylation, following host cell lysis, hints at a potential role for TgCAP in actin regulation during rapid egress. Taken together, these results prompted us to characterise the role of TgCAP in Toxoplasma biology.

Here we show that TgCAP is produced in two distinctly localised isoforms through alternative translation initiation: a membrane bound isoform, localised to the apical tip (longCAP) and a cytosolically dispersed isoform (shortCAP). Conditional knockout of TgCAP, using a second generation DiCre strain, identified an important function of TgCAP in some, but not all, actindependent processes. Invasion, egress, motility, correct daughter cell orientation and dense granule trafficking were all perturbed in CAP depleted parasites, while apicoplast inheritance was not. This suggests different spatial requirements for CAP in actin turnover within the cell. Furthermore, the characteristic rosette organisation of parasites in the vacuole was completely lost, but synchronicity of division was unaffected. Strikingly, while we observe rapid protein transfer only between two adjacent cells in a vacuole, all parasites remain connected through a decentralised residual body, potentially explaining why synchrony of division is unaffected. In the mouse in vivo infection model, CAP-depleted type I RH parasites display normal lethality, while CAP depletion renders type II Pru parasites avirulent, with markedly reduced cyst formation. Furthermore, the cytoplasmic isoform of TgCAP was sufficient for the infection of mice and the formation of latent stages in the brain, indicating that the apically localised CAP isoform provides only a small fitness benefit under the conditions tested here.

\section{Results \\ TgCAP contains two translational start sites which results in the production of two differentially localised protein isoforms.}

TgCAP was previously shown to localise to the apex of intracellular parasites and rapidly redistribute to the cytoplasm of extracellular parasites following host cell lysis (Lorestani et al., 2012). This suggested that TgCAP localisation may be influenced by post-translational modifications that enable CAP to regulate actin dynamics at different locations in the cell. TgCAP, and CAP from Neospora and Hammondia species contain a unique predicted $\mathrm{N}$ terminal extension that is not present in other Apicomplexa, such as Plasmodium (Fig 1A). The extension contains two predicted palmitoylation sites and CAP was identified in an analysis of palmitoylated proteins in Toxoplasma gondii (Foe et al., 2015). Furthermore, two phosphorylation sites in the $\mathrm{N}$-terminus of TgCAP are substantially phosphorylated upon ionophore-induced egress (Treeck et al., 2014). These phosphorylation events are dependent on the calcium-dependent kinase 3 (TgCDPK3) (Treeck et al., 2014), which has been shown to be important in mediating rapid exit from the host cell and is localised to the plasma membrane (Black et al., 2000; Garrison et al., 2012; Lourido et al., 2012; McCoy et al., 2012). Collectively, these observations allow for the possibility that re-localisation of CAP is important for egress and is mediated by dynamic post-translational modifications. To visualise TgCAP we expressed it as a GFP fusion. TgCAP-GFP localises to the apex of the parasite, as previously shown, but also to the cytoplasm of intracellular tachyzoites (Fig. 1B). We additionally demonstrated this dual localisation of TgCAP by C-terminally tagging the endogenous TgCAP locus with a HA epitope tag (Supplementary Fig. 1). To rule out any mislocalisation of the protein as a result of tagging, we expressed recombinant $\mathrm{TgCAP}$, spanning residues 37 to 203, and generated antibodies against TgCAP which confirmed the dual localisation (Fig 1C). Western blot analysis of parasite lysates revealed the presence of two bands close to the expected size of TgCAP, which are expressed at a constant level, relative to the Toxoplasma loading control, across the first 24 hours following host cell invasion (Fig. 1D). The dual localisation of CAP could be due to the expression of two isoforms from a single 
gene, through use of alternate translational start sites, as previously observed for protein kinase G (Brown et al., 2017). Indeed, sequence comparison of TgCAP to PfCAP reveals a second in-frame methionine at position 37 in Toxoplasma that aligns with the PfCAP start methionine (Fig. 1A). Two additional methionine residues, M71 and M161, are present in the CAP primary sequence. However, these are unlikely used as translational start sites as their products would lead to a truncated and potentially inactive CARP domain.

Translational start sites in eukaryotic mRNAs are preceded by a translation initiation sequence (Kozak, 1987a, 1987b). Consensus translation initiation sequences have been determined for many different organisms and are known as the Kozak sequence (Nakagawa et al., 2008). In Toxoplasma, the Kozak sequence was elucidated by identifying nucleotides commonly found in highly abundant proteins, such as SAG1 (Seeber, 1997). This Kozak sequence contains an adenine at the -3 position, relative to the start ATG, and was identified as the most important factor in ribosomal recognition of the start ATG (Seeber, 1997). Absence of an adenine can result in ribosome "leaky scanning" and translation from an internal ATG. We therefore analysed the translation initiation sequence around CAP's first (M1) and second (M37) putative translational start sites. The M1 translation initiation sequence conforms less with the Toxoplasma Kozak sequence than the sequence preceding M37; as the former is lacking an adenine at the -3 position (Fig. 1E). This suggested that alternative translation could lead to the generation of two CAP isoforms: longCAP, which is translated from the first start ATG, and shortCAP, which is translated from the second start ATG. To test this, we generated parasite strains that expressed either the WT sequence or variants where either the first (M1), or the second methionine (M37) of TgCAP was mutated to leucine, precluding their use as translational start sites (Fig. 1F). We used the endogenous promotor (i.e. 969 bp upstream of the first start ATG) and introduced the C-terminal HA-tagged TgCAP variants into the UPRT locus of the RH DiCre $\Delta k u 80 \Delta h x g$ prt parasite strain. To determine whether longCAP and shortCAP show differential localisation, as predicted by the presence of putative palmitoylation sites in longCAP, we analysed their subcellular localisation using the HA-tag. While WT CAP parasites showed the expected dual localisation, mutants expressing shortCAP showed exclusively cytoplasmic staining while those expressing longCAP showed predominantly apical staining, with some further signal throughout the parasite (Fig. 1G). As longCAP contains two putative palmitoylation sites not present in the shortCAP sequence, we next evaluated whether palmitoylation was important for the apical localisation of the long CAP isoform. We mutated the two cysteines in the N-terminus to alanine residues (CAP_C6,8A). CAP_C6,8A appeared cytosolic with no detectable accumulation of CAP at the apical end of the parasites (Fig. 1G). Western blot analysis of the HA-tagged TgCAP variants confirmed that WT CAP is identified as two distinct protein bands, which correlate with the predicted size for longCAP and shortCAP (23.9 and $20 \mathrm{kDa}$ ). Parasites expressing shortCAP displayed only the lower molecular weight band, while longCAP-expressing parasites only showed the higher molecular weight band. We observed that the protein levels of longCAP appear reduced compared to its isoform in parasites expressing WT CAP, whereas the shortCAP isoform shows an increase (Fig. 1H). As expected, despite CAP_C6,8A not being detected at the apical end of the parasite, both isoforms were detected by Western blot at levels comparable to parasites expressing WT CAP. Collectively, these results show that in Toxoplasma, and potentially closely related coccidian parasites of Hammondia and Neospora, CAP is produced as two differentially localised isoforms using alternative translational start sites and the apical localisation of the long isoform is likely palmitoylation dependent.

\section{Generation of a more stable RH DiCre $\Delta k u 80$ cell line}

While attempting to generate a conditional knock out (cKO) of CAP using the DiCre strategy, we observed a frequent loss of one of the DiCre subunits, resulting in dysfunctional floxed CAP parasite strains that lacked the ability to excise CAP. This is possibly because both DiCre subunits are driven by identical 5' and 3' UTRs, allowing for potential recombination in the $\Delta k u 80$ parental line, which possesses an increased efficiency of homologous recombination (Fox et al., 2009; Huynh \& Carruthers, 2009). To prevent loss of DiCre subunits, we generated 
a new DiCre construct, DiCre_T2A, that expresses the two DiCre subunits from a single promotor using T2A skip peptides (Kim et al., 2011). To further minimise the potential for loss of DiCre, we placed a chloramphenicol acetyltransferase (CAT) selectable marker between the two subunits (Fig. 2A). This would lead to the production of the two separate Cre subunits and the CAT selectable marker. We inserted this construct, into the modified KU80 locus of the RH $\Delta k u 80 \Delta h x g p r t$ strain (Huynh \& Carruthers, 2009) using CRISPR/Cas9. To test whether expression of the DiCre subunits in the resulting line, RH DiCre_T2A $\Delta k u 80 \Delta h x g p r t$, is stable over time, we integrated the loxP-KillerRed-loxP-YFP reporter construct used in Andenmatten et al., into the UPRT locus (Andenmatten et al., 2013). As expected, non-treated parasites express KillerRed which, upon RAP treatment, is excised and leads to expression of YFP (Fig. 2B). As extracellular stress can lead to increased loss of DiCre activity in the original DiCre line (M. Meissner, personal communication, 02.2019), we subjected the new DiCre parasite line (RH DiCre_T2A $\Delta k u 80 \Delta h \times g p r t)$ to frequent extracellular stress over the course of 65 days. On average, parasites were passaged every 2.3 days, leaving parasites extracellular for $\sim 32$ hours in the presence or absence of continuous chloramphenicol selection. We also simultaneously passaged the original DiCre line (Andenmatten et al., 2013) under standard, non-stressing, culturing conditions. The new DiCre_T2A line excision efficiency varied between 98 and $99 \%$ for replicates on day 1 and was maintained throughout the experiment with a maximal loss of $3 \%$ of excision efficiency, irrespective of the presence or absence of chloramphenicol selection, by day 65 (Fig. 2C). In contrast, the original DiCre line, cultured under standard non-stress conditions, lost $42 \%$ of excision capacity by day 65 (Supplementary Fig. 2), although this was only done as a single replicate. This shows that the second generation DiCre line, RH DiCre_T2A $\Delta k u 80 \Delta h x g p r t$, retains high excision capacity over long periods of time, even when exposed to extracellular stress.

TgCAP is important but not essential for in vitro growth and deletion can be largely restored by the short cytoplasmic isoform, but only partially by the membrane bound isoform.

To investigate CAP function, we generated a conditional knock out (cKO) of CAP using the DiCre strategy. Here, CAP with a C-terminal HA tag is flanked by two loxP sites, that recombine upon dimerisation of two split-Cre subunits, a process mediated by the small molecule rapamycin (RAP) (Andenmatten et al., 2013). To create the CAP conditional knockout line, we integrated a floxed, recodonised and HA-tagged CAP cDNA sequence into the endogenous locus of the RH DiCre $\Delta k u 80 \Delta h \times g$ prt line (Fig. $3 \mathrm{~A}$ ). Correct integration into the locus was confirmed by PCR (Fig. 3B). However, due to the DiCre issues detailed above, we were not able to successfully induce DiCre-mediated excision of the CAP gene. As the DiCre_T2A strategy demonstrated consistently high excision rates over time, during our testing with a reporter construct, we integrated the DiCre_T2A construct into the KU80 locus of the nonexcising floxed CAP parasite strain. This generated the parasite line $\mathrm{RH}$ DiCre_T2A DiCre $\Delta k u 80 \Delta h x g$ prt LoxPCAP-HA, called LoxPCAP hereafter. As expected, LoxPCAP displayed dual localisation by IFA (Fig. 3C). RAP treatment resulted in a complete loss of CAP $(\triangle \mathrm{CAP})$, as shown by IFA (Fig. 3C) and Western blot (Fig. 3D).

To assess the requirements of CAP and its isoforms for various Toxoplasma functions, we complemented LoxPCAP parasites with either wildtype, the short or the long CAP isoform by integration of HA-tagged variants into the UPRT locus to generate merodiploid lines (named LoxPCAPCAP, LoxPCAP ${ }^{\text {shortCAP }}$ and LoxPCAPlongCAP, respectively). We then excised the endogenous CAP copy by RAP treatment. Clones were subsequently obtained by limiting dilution and excision verified by PCR (Supplementary Fig. 3).

This resulted in parasite strains that express only the WT complemented form ( $\left.\triangle \mathrm{CAP}^{\mathrm{CAP}}\right)$, the short complemented form ( $\left.\triangle \mathrm{CAP}^{\text {shortCAP }}\right)$ or the long complemented form $\left(\triangle \mathrm{CAP}{ }^{\text {longCAP }}\right)$. We confirmed the differential localisation and translation of these isoforms by IFA (Fig. 3E) and Western blot (Fig. 3F). No differences in the protein levels of the two CAP isoforms could be 
observed in the CAP complemented strain ( $\triangle \mathrm{CAP} C A P)$ over the first 24 hours following host cell invasion, relative to the loading control (Supplementary Fig. 4).

To reliably quantify the contribution of CAP, and its two isoforms independently, to the lytic cycle, we performed a competition assay in which growth of $\triangle C A P^{C A P}$ was compared to $\triangle C A P$, $\triangle \mathrm{CAP} \mathrm{P}^{\text {shortCAP }}$ and $\triangle \mathrm{CAP} \mathrm{P}^{\text {longCAP. }}$. To do so, we integrated an mCherry expressing cassette into the $\Delta k u 80$ locus, replacing the DiCre_T2A cassette in each of these lines. Note, the DiCre_T2A cassette was no longer required because CAP had already been excised in these parasites. After 15 days in growth competition with $\triangle \mathrm{CAP} C A P, \triangle \mathrm{CAP}$ parasites were largely depleted $(>97.3 \%)$ from the population, while $\triangle \mathrm{CAP}{ }^{\text {shortCAP }}$ showed a reduction of only $4 \%$. In contrast, $\triangle C A P^{\text {longCAP }}$ showed an intermediate level of depletion $(33.3 \%)$. These phenotypes were exaggerated after 30 days in culture; $\triangle$ CAP parasites were largely depleted $(>99.9 \%)$, $\triangle C A P^{\text {shortCAP }}$ showed a reduction of $12.4 \%$, and $\triangle C A P^{\text {longCAP }}$ growth was reduced by $43.1 \%$ relative to $\triangle C A P^{C A P}$ (Fig. $4 A$ ). Collectively, these findings demonstrate that CAP plays an important but non-essential role in cell culture and that its function can be largely restored by the short CAP isoform but only partially by the long isoform.

\section{TgCAP contributes to motility, invasion, egress and dense granule trafficking}

While the competition assays highlight the importance of CAP during the in vitro lytic cycle, they do not clarify which step of the cycle is affected. To test whether the growth differences between the lines were merely a result of differences in parasite replication rates, we counted the parasites per vacuole for each parasite line. No significant differences were observed (Supplementary Fig. 5). As CAP is a predicted actin regulator, we next focused our phenotypic analysis on Toxoplasma processes for which actin is known to be important, such as motility, egress, invasion and dense granule trafficking (Heaslip et al., 2016; Periz et al., 2017; Whitelaw et al., 2017).

In the absence of CAP $(\triangle \mathrm{CAP})$ significantly fewer parasites are able to initiate gliding motility in 3D motility assays (Leung et al., 2014) compared to the RH strain (Fig. 4B). In the motile population, trajectory displacement, trajectory length and maximum achieved speeds were all significantly reduced, although mean speed was not significantly different (Fig. 4B). To determine which isoform(s) contribute to parasite $3 \mathrm{D}$ motility, the motility parameters of $\triangle \mathrm{CAP} C A \mathrm{P}, \triangle \mathrm{CAP}^{\text {shortCAP }}$ and $\triangle \mathrm{CAP} \mathrm{P}^{\text {longCAP }}$ were compared. Both $\triangle \mathrm{CAP}$ shortCAP and $\triangle \mathrm{CAP} \mathrm{P}^{\text {long } C A P}$ parasites showed similar levels of motility initiation, track displacement, track lengths and speed compared to $\triangle$ CAP $^{\mathrm{CAP}}$ (Supplementary Fig. 6). These data indicate that CAP plays a role in initiation of motility and in controlling speed and track length once motile. Complementation with single isoforms shows that initiation of motility can be rescued by either CAP isoform and, once motile, either can maintain speed and track length.

As invasion and egress of host cells rely on active motility, we next compared invasion efficiency of $\triangle \mathrm{CAP}$ and the different complementation lines. $\triangle \mathrm{CAP}$ showed a significant reduction of invasion capacity (50.2\% reduction compared to WT complemented lines), which was restored by both the short and long isoforms (Fig. 4C). We also performed egress assays in the presence of BIPPO, which results in a strong calcium response in Toxoplasma parasites causing synchronised and rapid egress from host cells (Howard et al., 2015). $\triangle$ CAP parasites showed a substantial delay in egress from host cells at $30 \mathrm{~s}$ after induction $(73.1 \%$ less egress in $\triangle \mathrm{CAP}$ compared to $\triangle \mathrm{CAP} \mathrm{PAP}^{\mathrm{C}}$ ), while at 2 minutes the majority of parasites have egressed (10.6\% less egress in $\triangle$ CAP compared to $\triangle$ CAPCAP) (Fig. 4D). This defect was fully restored in $\triangle C A P^{\text {shortCAP }}$ parasites, while $\triangle C A P^{\text {longCAP }}$ showed slightly lower levels of egress after $30 \mathrm{~s}$ of treatment but by $60 \mathrm{~s}$ were indistinguishable from WT or short CAP complemented lines. We also observed live egress events in which $\triangle \mathrm{CAP}$, following host cell egress, showed decreased

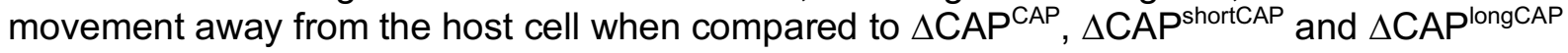
(Videos 1-4). 
A recent study revealed that directed dense granule transport is dependent on filamentous actin (Heaslip et al., 2016). Therefore, dense granule trafficking was assessed in $\triangle C A P$ and the three complemented lines. We measured the run frequency (\# of directed runs/parasite/minute), run length and velocity of directed motions. (Fig. 4E). Both the mean run length and the mean velocity were significantly increased in $\triangle \mathrm{CAP}(34.8 \%$ and $52.9 \%$, respectively), but not in either of the complements. However, the frequency of directed runs was reduced in $\triangle \mathrm{CAP}(14.8 \%)$, but did not reach significance. These results indicate that both CAP isoforms play a supporting role in dense granule trafficking and that, upon CAP deletion, dense granules are trafficked further distances at higher speed. Collectively these data show that CAP plays a role in the actin-dependent processes described above and that either isoform is able to fulfil these CAP functions.

\section{$\triangle$ CAP parasites display a defect in efficient cell-cell communication}

Actin, formin 3 and myosin I and $\mathrm{J}$ have been shown to be important for both parasite rosette organisation and cell-cell communication, as assessed by measuring the rapid transfer of reporter proteins between individual parasites in a vacuole (Frénal, Jacot, et al., 2017; Periz et al., 2017; Tosetti et al., 2019). Upon CAP deletion, we observed a complete loss of the characteristic rosette formation normally seen in Toxoplasma. This aberrant phenotype was fully rescued upon complementation with either WT CAP or the short CAP isoform (Fig. 5A). $\triangle C A P^{\text {longCAP }}$ displayed partial restoration with $44.9 \%$ of parasites forming phenotypically normal rosettes while the remainder were disorganised within the vacuole. This was also shown by scanning electron images of infected human fibroblasts in which the host cell and the vacuole membrane was removed ("unroofed") as previously described (Magno et al., 2005) (Supplementary Fig. 7).

It was previously shown that mutant parasite lines which lost rosetting capacity also lost the rapid transfer of reporter proteins (called RTORP hereafter) between them. This has been established by photobleaching of individual parasites in a vacuole, which express a fluorescent reporter protein. Under normal conditions, all parasites in a vacuole contribute to the fluorescence recovery of the photobleached parasite (Frénal, Jacot, et al., 2017; Periz et al., 2017; Foe et al., 2018; Tosetti et al., 2019). Such transfer of reporter proteins has previously been used as a readout for cell-cell communication and, by extension, parasite connectivity (Frénal, Jacot, et al., 2017; Periz et al., 2017). To examine whether loss of cell-cell communication also accompanied the disrupted rosetting observed in $\triangle \mathrm{CAP}$ parasites, we performed fluorescence recovery after photobleaching (FRAP) experiments on $\triangle C A P$ and its complementation lines. We chose vacuoles that contained 8 parasites/vacuole as at this stage parasites organise in rosettes and individual cells can be easily monitored. We bleached one parasite in the vacuole and recorded both the recovery of fluorescence in the bleached parasite and the fluorescence levels of all other parasites in the vacuole. As expected, the WTcomplemented $\triangle \mathrm{CAP}^{\mathrm{CAP}}$ photobleached parasites display rapid recovery of fluorescence to which, in most cases, all parasites in the vacuole appear to contribute (Fig. 5Bi, 5C and Video 5). Conversely, $\triangle$ CAP photobleached parasites were supported in their rapid fluorescence recovery predominantly by just one other parasite, usually the parasite closest to the bleached cell, resulting in slow recovery (Fig. 5Bii, 5C and Video 6). In some cases, no recovery was observed in $\triangle$ CAP parasites.

Loss of rapid protein transfer between parasites could be explained by a structural disruption of inter-parasite connections provided by the residual body. However, in addition to the residual body, an intravacuolar network (IVN) of tubule-like structures is present in the parasitophorous vacuole. Because of its tubular structure, it could also be involved in cell-cell communication. To determine whether the observed defect in the RTORP was dependent on the presence of the IVN, we performed a FRAP assay in RH $\Delta k u 80 \Delta$ gra2 parasites (Rommereim et al., 2016) where the IVN fails to form but parasites still organise in rosettes. The results show that RTORP between parasites was not negatively affected (Fig. 5Biii and Video 7). The IVN, therefore, is unlikely involved in cell-cell communication. 
To test if CAP deletion leads to a defect in cell-cell communication directly, or whether this is a direct consequence of the inability to form rosettes, we tested whether the RTORP varied between rosetting $(r)$ and non-rosetting parasites $(n r)$ of the $\triangle C A P^{\text {longCAP }}$ line. These experiments revealed that if $\triangle C A P^{\text {longCAP }}$ parasites rosette, they show normal RTORP between all cells in the vacuole, while non-rosetting parasites display the same defect as the $\triangle$ CAP line, with fluorescence recovery from only one other parasite in the vacuole (Fig. 5C). Interestingly, $\Delta$ gra2 vacuoles showed a slight increase in the frequency of photobleach recovery from multiple parasites, but we have not further investigated this phenomenon here. The connectivity of parasites was also assessed based on the percentage of recovery after photobleaching. As expected, $\triangle \mathrm{CAP}$ and non-rosetting $\triangle \mathrm{CAP} \mathrm{P}^{\text {longAP }}$ photobleached parasites recovered significantly less fluorescence than their rosetting counterparts (Fig. 5D).

Next, we sequentially photobleached individual $\triangle$ CAP parasites in a vacuole and identified which parasites appear to be physically connected based on the transfer of mCherry (Fig. 5E). This revealed that only parasite pairs in close proximity are rapidly communicating. Collectively the results show that CAP deletion leads to a loss of both rosetting and the rapid transfer of reporter proteins between more than two parasites. Furthermore, RTORP appears to be dependent on parasite rosette organisation, but not on presence of the IVN.

402

403

404

405

406

407

408

409

410

411

412

413

414

415

416

417

418

419

420

421

422

423

424

$\triangle$ CAP parasites have a defect in daughter cell orientation but not in synchronised division or apicoplast inheritance

Previously, cell-cell communication has been hypothesised to control synchronous division between parasites within the same vacuole (Frénal, Jacot, et al., 2017). To determine whether $\triangle$ CAP parasites display phenotypes previously observed for mutants with defective cell-cell communication, we used IMC3 antibodies to visualise synchronicity of forming daughter cells (Fig. 6A). Surprisingly, $\triangle \mathrm{CAP}$ vacuoles exhibited synchronous division with no significant difference to the WT complemented line (Fig. 6B). IMC3 staining was also used to assess daughter cell orientation. While in WT CAP complemented parasites $94.6 \%$ of daughter cells grew in the same orientation, a significant defect was observed in $\triangle \mathrm{CAP}$ and $\triangle \mathrm{CAP}$ longCAP strains; only $42.9 \%(\triangle \mathrm{CAP})$ and $67.7 \%\left(\triangle \mathrm{CAP} \mathrm{P}^{\text {longCAP }}\right)$ of daughter cells orientated in the same direction. $\triangle$ CAPshortCAP parasites were able to largely overcome this defect, with $89.5 \%$ of daughter cells growing in the same orientation (Fig. 6C). Such improper orientation of daughter cells following budding raises the possibility of improper organelle segregation too. To test this, we looked at apicoplast segregation, another actin-dependent process (Andenmatten et al., 2013; Jacot et al., 2013). Using streptavidin as a marker for the apicoplast, we identified no significant differences in apicoplast inheritance rates between $\triangle \mathrm{CAP}{ }^{\mathrm{CAP}}$ and $\triangle \mathrm{CAP}$ strains (Supplementary Fig. 8). These data show that despite a loss of rosette organisation and RTORP between parasites in a $\triangle \mathrm{CAP}$ vacuole, as well as disordered daughter cell orientation, parasite division remains synchronous. Furthermore, while CAP is supporting many actindependent processes in Toxoplasma, it appears dispensable for apicoplast division.

\section{CAP deletion leads to the formation of a decentralised residual body in which all parasites remain connected, despite loss of RTORP}

The observed synchronicity of division in the $\triangle \mathrm{CAP}$ strain, despite loss of RTORP, was unexpected. It suggested that synchronicity is either independent of the residual body, or that $\triangle \mathrm{CAP}$ parasites actually maintain connections that allow flow of proteins or metabolites to synchronise divisions. To investigate this, we established a connectivity map of parasites in a vacuole using correlative light and electron microscopy (CLEM). This allowed us to first analyse connectivity of parasites based on FRAP analysis, and then reconstruct a 3D electron microscopy image of the parasites and their connections by focused ion beam scanning electron microscopy (FIB SEM). contribute to recovery of a photobleached parasite (Fig. 7A) and a normal residual body is 
formed, connecting all parasites in the vacuole (Fig. 7B and Video 8), with one tubular extension extending away from the residual body with no apparent connections at the distal end. In contrast, for the $\triangle \mathrm{CAP}$ strain, fluorescence recovery of the photobleached parasite was parasites are connected, we analysed FIB SEM images obtained from the photobleached vacuole in Fig. 7C. This revealed membrane bound tubular connections of approximately 300 $\mathrm{nm}$ thickness between parasites, despite the aberrant transfer of mCherry and loss of rosetting (Fig. 7Di and ii). Following the lumen of the connections across 3 dimensions demonstrates that all parasites in the vacuole are connected by these tubular connections, likely representing a decentralised residual body that forms as a result of an inability to keep the posterior ends of the parasites in close proximity (Fig.7D.iii, Supplementary Fig. 9 and Video 9). Correlation of the FRAP data with the FIB SEM images showed that rapid transfer of mCherry was always between parasites in close proximity at their basal ends.

While $\triangle$ CAP parasites appear to be connected via a decentralised residual body, it could be that transfer of material through these connections is limited by physical barriers, such as the mitochondria which have previously been observed in the residual body (Frénal, Jacot, et al., 2017). Close examination of the residual body connections showed only one such connection contained two mitochondria and a constriction of the decentralised residual body lumen to $\sim 50$ $\mathrm{nm}$, while all other connections appeared free of large physical barriers, indicating that this is unlikely an explanation for the lack of RTORP between parasites. However, we observed a complex network of tubules and sheet-like structures, likely representing the endoplasmic reticulum, in the connections (Fig. 7E) (Puhka et al., 2012; Schroeder et al., 2019; Tomavo et al., 2013; West et al., 2011). One of these tubular structures, tracked in three dimensions, was shown to enter multiple parasites via the basal pole, suggesting a possible continuum between them, which was also observed in then WT CAP complemented parasites. This has not been further examined here but could contribute to exchange of material between parasites.

Collectively, while CAP is important for rosette organisation of parasites, it is not essential for forming and sustaining a residual body with connectivity to all parasites in the vacuole. Furthermore, it shows that the RTORP is not an indicator of parasite connectivity.

\section{Deletion of CAP results in completely avirulent type II parasites, but not in the type I RH strain.}

The short CAP isoform complements most phenotypes in cell culture while the long CAP isoform, in most cases, only shows a partial rescue. This raises the question about the evolutionary roles of the two different isoforms. To better discriminate the functions of the short and longCAP isoforms, we wanted to examine their respective roles in natural infections, where parasites encounter a number of additional stresses, including shear stress and the immune system. Accordingly, we addressed the essentiality of CAP and its isoforms in mouse infections. We hypothesised that if both isoforms are essential for parasite survival in a natural host, loss of either of the two isoforms would manifest in a fitness cost. First, we injected male C57BL/6 mice with $\sim 25 \Delta \mathrm{CAP}$ or $\triangle \mathrm{CAP} \mathrm{CAP}^{\mathrm{C}}$ tachyzoites of the virulent RH strain, and monitored them over the course of 10 days. In both instances mice began to succumb to infection after 9 days, indicating that, despite the motility and rosetting phenotypes, CAP depletion in a type I $\mathrm{RH}$ background still results in high virulence in mice (Fig. 8A). As $\mathrm{RH}$ parasites have frequently been associated with hypervirulence and do not form cysts in vivo, we next generated $\triangle C A P$ parasites and complemented versions in the type II Pru strain. In stark contrast to the RH line, upon injection of $\sim 50,000$ tachyzoites, Pru $\triangle$ CAP parasites showed no virulence in mice while the Pru $\triangle C A P^{C A P}$, Pru $\triangle C A P^{\text {shortCAP }}$, and Pru $\triangle C A P^{\text {longCAP }}$ complemented parasites led to a lethal infection, with most mice succumbing to the parasites 8-10 days post-infection (Fig. 8B). These data show that expression of either individual isoform is sufficient to cause a lethal infection. Next, to look at formation of tissue cysts, which leads to a chronic infection, we injected a lower dose of $\sim 5,000$ tachyzoites of the Pru lines into mice. The majority of mice survived until day 32 post-infection, although $2 \triangle \mathrm{CAP}^{\mathrm{CAP}}$-infected mice and $1 \Delta \mathrm{CAP}^{\text {shortCAP }}$-infected mouse died 
before reaching this end-point. At day 32, the mice were sacrificed, brain samples were collected and serum tested for anti-Toxoplasma antibodies, confirming that all mice were successfully infected with Toxoplasma (Fig. 8C). Both the Pru $\triangle$ CAP and Pru $\triangle$ CAPlongCAP infections demonstrated significantly lower cyst loads compared to Pru $\triangle C A P^{C A P}$ and Pru

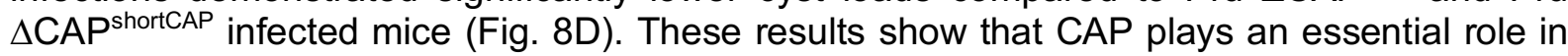
the virulence of the type II Pru parasite strain, but not the type I RH strain. Moreover, while the short CAP isoform is able to fulfil all functions of TgCAP in cell culture and in the mouse model, the long isoform, despite its ability to complement most phenotypes to at least $\sim 50 \%$ of WT complement levels, has a significant defect in establishing a chronic infection at the infectious dose used here.

\section{Discussion}

Toxoplasma actin is important for a range of cellular processes, from organelle segregation and cell-cell communication, to gliding motility: a crucial factor in parasite dissemination (Andenmatten et al., 2013; Egarter et al., 2014; Periz et al., 2017). Despite the key role of actin in parasite biology, our understanding of actin dynamics and its regulation remains incomplete. CAP is a ubiquitous protein with a conserved role in regulating actin dynamics. In this study, we established that Toxoplasma CAP is expressed by an alternative translation initiation site, giving rise to two independent isoforms: shortCAP and longCAP. Through sequence alignment we identified that the alternate translation initiation site is conserved only within Toxoplasma, Neospora and Hammondia. These are all members of the Toxoplasmatinae, a subfamily of the Apicomplexa phylum, suggesting that while CAP is present in all apicomplexa, the long isoform is specific to Toxoplasma and its closest relatives. Here we investigated the role of both CAP isoforms in the Toxoplasma lytic cycle. Through in vitro competition assays, we show that complementation with shortCAP is enough to overcome the majority $(87.6 \%)$, but not all, of the growth defect associated with CAP depletion. This suggests that longCAP is performing a specific function important for parasite fitness, which shortCAP is unable to compensate for. Furthermore, longCAP complementation restored $56.9 \%$ of the growth defect, arguing for a degree of functional overlap between the two isoforms. Our experiments have not uncovered a phenotype that only longCAP can rescue, which would have revealed a unique function. Its apical localisation, however, makes it temping to speculate a function in actin regulation during invasion, which is a phenotype it can fully rescue. It could be that higher actin turnover is required at the apex and the concentration of longCAP here is fulfilling this need by influencing G-actin levels. Indeed, it has previously been hypothesised in other organisms that increased local concentration of CAP results in the sequestration of actin monomers (Ono, 2013). However, providing evidence for this hypothesis is limited by the fact that the shortCAP isoform can fully rescue all phenotypes in cell culture under the conditions tested. Given that cell culture assays do not fully represent the environment Toxoplasma normally encounters, we aimed to tease apart the functions of the two isoforms in mice. Surprisingly, even here the short isoform appears able to compensate for the lack of longCAP. This suggests that even under conditions encountered in the natural host, the longCAP isoform plays only a minor role, although dosage effects or routes of infection may well be confounding factors when assessing virulence of the different strains. Competition experiments between the mutants in vivo may help to tease apart the independent importance of the isoforms in the future. However, from the in vitro competition assays it can be predicted that parasites expressing two isoforms fare better than those expressing just one isoform. We cannot rule out that the differences in proteins levels of longCAP and shortCAP in the single isoform producing lines are affecting the phenotypic observations. However, both isoforms are able to rescue most phenotypes indicating that the protein levels do not appear to substantially affect CAP function.

Despite being dispensable for type I RH parasite virulence, CAP is essential for type II Pru parasite virulence in mice, and complementation with either shortCAP or longCAP restored lethality. At lower, non-lethal doses, longCAP complementation led to markedly reduced cyst formation in the brain. The underlying basis for this has not been explored here and it could be 548 that it is the reduced fitness of the strain, rather than stage conversion phenotype, that causes 
549 a reduction of parasites reaching the brain. Therefore, the observed virulence of the longCAP 550 complemented strain is possibly due to the high dosage used, allowing the parasite to 551 proliferate at high enough rates to overwhelm the immune system. Nevertheless, the basis for reduced fitness of $\triangle \mathrm{CAP}$ and $\triangle \mathrm{CAP}{ }^{\text {longCAP }}$ parasites in the murine infection model is likely multifactorial and we hypothesise that other actin-dependent processes that we have not assayed here could also contribute to the reduced virulence in mice.

The most pronounced phenotype of CAP deletion is loss of rosetting: the highly symmetrical physical distribution of parasites within the vacuole. Previous studies on actin, myosin I, myosin $\mathrm{J}, \mathrm{ADF}$ and formin 3 have suggested that the actomyosin motor is important for rosetting (Frénal, Dubremetz, et al., 2017; Haase et al., 2015; Periz et al., 2017; Tosetti et al., 2019). Our $\triangle$ CAP data further supports this hypothesis. Interestingly, complementing $\triangle$ CAP parasites with longCAP restored rosetting in just under half of all vacuoles, with the remainder appearing as equally disordered as the $\triangle \mathrm{CAP}$ vacuoles. Despite this mixed population, we did not observe vacuoles with a combination of organised and disorganised parasites, suggesting rosetting is a binary outcome; either all parasites in a vacuole are connected by a central residual body, or not. This may suggest that the ability to produce highly organised rosettes is established during the first round of cell division when the residual body forms. This mixed population of rosetting and non-rosetting parasites, in an isogenic strain, gave us a unique opportunity to determine whether rosetting is important for efficient intravacuolar cell-cell communication. Using longCAP complemented parasites, we show that in rosetting vacuoles there is efficient RTORP with all parasites able to transfer material to the bleached parasite. Within the same population, non-rosetting vacuoles displayed severe defects in the RTORP with parasites seemingly only communicating in pairs. However, despite apparently only communicating in pairs, all parasites in the vacuole remained synchronised in their stage of replication. This is different to other studies which suggested that defects in RTORP between parasites leads to asynchrony in division (Frénal, Jacot, et al., 2017; Periz et al., 2017). Our results show that neither RTORP between daughter cells nor the formation of a rosette are predictors for synchronicity of division.

The synchronous division in $\triangle$ CAP parasites could be explained by our FIB SEM results. Despite their disorganised appearance and loss of rapid cell-cell communication, $\triangle \mathrm{CAP}$ parasites are still connected by a decentralised residual body. This connection, although not facilitating rapid transfer of proteins between parasites that are further apart, could allow for slow or minimal transfer of proteins which is sufficient to synchronise divisions. An alternative hypothesis could be that metabolites, not proteins, are required to synchronise divisions, and their diffusion through the decentralised residual body is quicker. It is also a possibility that the seemingly continuous ER in the residual body, observed here connecting most if not all parasites, could contribute to synchronicity of division. Whatever the basis for synchronicity is, rosetting and rapid cell-cell communication are not essential and their analysis cannot be reliably used to predict whether connections between parasites exist. Recently, Foe et al reported that upon deletion of $\mathrm{ASH} 4$, a serine hydrolase, parasites were only connected in pairs but retained synchronicity of division (Foe et al., 2018), similar to our results. An explanation could be that ASH4 mutants display a phenotype similar to the CAP phenotype described here, where, despite the loss of RTORP, parasites remain connected by a decentralised residual body, allowing synchronicity of division.

In summary, our results strongly support an actin regulatory role for CAP in Toxoplasma. Interestingly, actin dependent processes were affected to differing extents in $\triangle$ CAP parasites, such as rosetting being completely lost while apicoplast inheritance was unaffected. This surely reflects the different spatial requirements for actin turnover within a cell. It is likely that the local concentration of actin, actin binding proteins such as the formins and the different myosins facilitate this. The results obtained here also leave open a few questions that are interesting to study in the future. How does actin help to initiate and maintain the centralised residual body? How are ER connections between parasites in a decentralised residual body 
maintained and what is the function of this connection? What is the functional role of longCAP with its high concentration at the apex of the parasite? Why are some actin dependent processes completely reliant on CAP while others are not? Each of these questions will require careful analysis and the cell lines described here will likely provide useful tools to investigate these in the future.

\section{Materials and methods \\ Plasmid construction}

All primers used in this study are listed in Supplementary File 1. All synthetic DNA used in this study is listed in Supplementary File 2.

To generate the CAP-GFP fusion plasmid, pUPRT_CAP_GFP, the Toxoplasma CAP gene (TGME49_310030) 5'UTR was amplified from genomic DNA using primer pair P1/P2 and Gibson assembled (Gibson et al., 2010) with a synthetic CAP cDNA-Xmal-eGFP sequence (GeneArt strings, Life Technologies, Massachusetts, United States) into BamHI and Pacl digested UPRT targeting vector pUPRT-HA (Reese et al., 2011).

To generate the CAP cKO plasmid, pG140_CAP_cKO_LoxP111, the CAP 5'UTR with a LoxP site inserted $111 \mathrm{bp}$ upstream of the $C A \bar{P}$ start codon, and a recodonised CAP CDNA-HA sequence, were synthesised (GeneArt strings, Life Technologies). These DNA fragments were Gibson cloned (Gibson et al., 2010) into the parental vector p5RT70loxPKillerRedloxPYFP-HX (Andenmatten et al., 2013) which had been digested using Apal and Pacl, creating an intermediate plasmid. Next, the CAP 3'UTR was amplified from genomic DNA using primer pair P3/P4 while mCherry flanked by GRA gene UTRs was amplified from pTKO2c (Caffaro et al., 2013) using primers P5/P6. These PCR products were Gibson cloned (Gibson et al., 2010) into the Sacl digested intermediate plasmid to create pG140_CAP_cKO_LoxP111.

To generate pUPRT_CAP, the CAP 5'UTR was amplified from genomic DNA using primer pair $\mathrm{P} 1 / \mathrm{P} 2$. This DNA fragment and a synthetic CAP cDNA-BamHI-HA sequence (GeneArt strings, Life Technologies), were Gibson cloned (Gibson et al., 2010) into BamHI and Pacl digested UPRT targeting vector pUPRT-HA (Reese et al., 2011).

To generate pUPRT_CAP_C6,8A, the CAP 5'UTR was amplified from genomic DNA using primer pair P1/P2 and, alongside a synthetic CAP CDNA-HA sequence with C6,8A mutations (GeneArt strings, Life Technologies), was Gibson cloned (Gibson et al., 2010) into BamHI and Pacl digested UPRT targeting vector pUPRT-HA (Reese et al., 2011).

To generate pUPRT_CAP_M1L, pUPRT_CAP was amplified with primer pair P7/P8 to introduce the M1L point mutation.

To generate pUPRT_CAP_M37L, pUPRT_CAP was amplified with primer pair P9/P10 to introduce the M37L point mutation.

To generate pG140_DiCre, the plasmid containing DiCre_T2A, two synthetic DNA fragments were produced containing Ku80 homology region-alpha-tubulin promoter-FRB-Cre60-T2Achloramphenicol resistance cassette-T2A-FKBP-Cre59-SAG1 3'UTR-Ku80 homology region (gBlock gene fragments, Integrated DNA Technologies, lowa, United States). These DNA fragments were Gibson cloned (Gibson et al., 2010) (into Apal and Sacl digested parental vector, p5RT70loxPKillerRedloxPYFP-HX (Andenmatten et al., 2013)

To generate plasmids expressing CAS9 and a single guide RNA (sgRNA), we used plasmid pSAG1::CAS9-U6::sgUPRT as a backbone (Addgene plasmid \#54467) (Shen et al., 2014). The plasmid was amplified with primer P11 and another containing a sgRNA to replace the UPRT-targeting sgRNA. Guide RNA sequences were selected using the Eukaryotic Pathogen CRISPR gRNA Design Tool (Peng \& Tarleton, 2015). All sgRNA-expressing plasmids used in 
this study were generated by this strategy. The sgRNA-containing primers are listed in Supplementary File 1.

For creation of plasmids with multiple sgRNAs, first, two separate vectors, each with a sgRNA, were generated as described above using the parental vector pSAG1::CAS9-U6::sgUPRT. Then, primers P12/P13 were used to amplify one of the sgRNA regions which was Gibson cloned (Gibson et al., 2010) into the other $\mathrm{Kpnl}$ and $\mathrm{Xhol}$ digested plasmid, creating a multiple sgRNA plasmid.

\section{Culturing of parasites and host cells}

T. gondii tachyzoites were cultured in human foreskin fibroblasts (HFF) and in Dulbecco's modified Eagle's medium (DMEM) with GlutaMAX (Invitrogen, California, United States) supplemented with $10 \%$ fetal bovine serum and maintained at $37^{\circ} \mathrm{C}$ with $5 \% \mathrm{CO}_{2}$.

\section{Generation of parasite lines}

To generate the conditional CAP knockout strain (RH DiCre $\triangle k u 80 \Delta h x g p r$ LLoxCAP-HA, referred to here as LoxPCAP), first, the linearised plasmid pG140_CAP_cKO_LoxP111, carrying the $H X G P R T$ cassette, was transfected into the $\mathrm{RH}$ DiCre $\Delta k u \overline{80} \Delta h \times \bar{g} p r t$ strain (Andenmatten et al., 2013). Resistant parasites were cloned. Next, the DiCre conditional knockout function of the strain was restored. The linearised pG140_DiCre plasmid, carrying the chloramphenicol resistance cassette and homology with the Ku80 UTRs, was transfected into the strain. To increase Ku80-specific insertion efficiency, a plasmid with multiple Ku80targeting sgRNAs was generated as described above using primer pairs P11/P14 and P11/P15. Resistant parasites were cloned. Integration into the CAP endogenous locus was confirmed using primer pairs P16/P17 and P18/P19. Replacement of CAP gDNA was confirmed using primers P20/P21. Excision of the floxed CAP sequence was confirmed with primer pairs P16/P20.

To complement the LoxPCAP strain with CAP-expressing constructs, the linearised plasmid pUPRT_CAP, pUPRT_CAP_M1L or pUPRT_CAP_M37L was transfected alongside pSAG1::CAS9-U6::sgUPRT. 5-fluorodeoxyuridine (FUDR) resistant parasites were cloned.

CAP was subsequently excised from the above strains by addition of $50 \mathrm{nM}$ rapamycin in DMSO for $4 \mathrm{hr}$ at $37^{\circ} \mathrm{C}$ with $5 \% \mathrm{CO} 2$, before washout, and excised parasites were cloned. Next, to aid with experimentation, an mCherry fluorescent construct was integrated into the Ku80 locus, replacing the present DiCre_T2A construct. mCherry flanked by GRA UTRs was amplified from pG140_CAP_cKO_LoxP111 using primer pair P22/P23 which also carries 30 bp homology with the $K u 80$ locus. To increase Ku80-specific insertion efficiency, a plasmid with multiple Ku80-targeting sgRNAs was generated as described above using primer pairs P11/P24 and P11/P25 and co-transfected with the PCR product. A population of parasites expressing mCherry were sorted by flow cytometry using a BD Influx cell sorter (BD Biosciences). Parasites were subsequently cloned, generating the strains $\triangle C A P, \triangle C A P^{C A P}$, $\triangle \mathrm{CAP}^{\text {shortCAP }}$ and $\triangle \mathrm{CAP} \mathrm{P}^{\text {longCAP. }}$.

The Pru $\Delta k u 80 \Delta$ cap strain was generated by amplifying the $H X G P R T$ resistance cassette from the pG140_CAP_cKO_LoxP111 plasmid using primer pair P26/P27. To direct insertion of the PCR product to the $C \overline{A P}$ locus, a plasmid with multiple $C A P$-targeting sgRNAs was generated as described above using primer pairs P11/P28 and P11/P29 and co-transfected with the PCR product. Resistant parasites were cloned. To complement the Pru $\Delta k u 80 \Delta$ cap strain with CAPexpressing constructs, the linearised plasmid pUPRT_CAP, pUPRT_CAP_M1L or pUPRT_CAP_M37L was transfected alongside pSAG1::CAS9-U6::sgUPRT. FUDR resistant parasites were cloned.

To generate fluorescent $\triangle$ gra2 parasites for FRAP experimentation, mCherry flanked by GRA UTRs was amplified from pG140_CAP_cKO_LoxP111 using primer pair P30/P31 with UPRT 
locus overhangs. This PCR product was co-transfected with pSAG1::CAS9-U6::sgUPRT into the RH $\Delta k u 80 \Delta g r a 2$ strain (Rommereim et al., 2016). An FUDR resistant population was

The RH DiCre_T2A $\Delta k u 80 \Delta h x g p r t$ line was generated by integrating the DiCre construct into the Ku80 locus in RH $\Delta k u 80 \Delta h x g$ prt parasites (Huynh \& Carruthers, 2009). The DiCre construct was amplified from pG140_DiCre using primer pair P32/P33 which also carries 30 bp homology with the Ku80 locus. To increase Ku80-specific insertion efficiency, a plasmid with a Ku80-targeting sgRNA was generated as described above using primer pairs P34/P11 and co-transfected with the PCR product. Resistant parasites were cloned.

To assess the efficiency of rapamycin-dependent excision of the DiCre strains, the Killer Red gene-swap construct was amplified from p5RT70loxPKillerRedloxPYFP-HX (Andenmatten et al., 2013) using primer pair P35/P36. This PCR product was co-transfected with pSAG1::CAS9-U6::sgUPRT for targeted insertion into the UPRT locus. FUDR resistant parasites were cloned.

To generate a CAP C-terminal endogenous HA-tagged line, a synthetic DNA repair template was produced containing CAP gDNA (a section of which is recodonised), a HA tag and the CAP 3'UTR (gBlock gene fragments, Integrated DNA Technologies). The DNA repair template was amplified using primer pair P37/P38. To direct insertion of the PCR product to the CAP locus, a plasmid with a single CAP-targeting sgRNA was generated as described above using the primer pairs P39/P11 and was co-transfected with the PCR product into RH $\Delta k u 80$ parasites. A population of parasites expressing the co-transfected Cas9-GFP-containing plasmid were sorted by flow cytometry using a BD Influx cell sorter (BD Biosciences). $\sim 70 \%$ of these parasites expressed the HA peptide by IFA.

\section{Parasite transfection and selection}

To generate stable transformants, $0.5-1 \times 10^{7}$ freshly lysed parasites were transfected with either $25 \mu \mathrm{g}$ of linearised template DNA, $5 \mu \mathrm{g}$ of linearised template DNA and $20 \mu \mathrm{g}$ of a corresponding gRNA-specific CRISPR/CAS9 plasmid or template DNA produced from 1 ethanol precipitated PCR and $20 \mu \mathrm{g}$ of a corresponding gRNA-specific CRISPR/CAS9 plasmid. Selection on the basis of 5 -fluorodeoxyuridine $(20 \mu \mathrm{M})$, mycophenolic acid $\left(25 \mu \mathrm{g} \mathrm{ml}^{-1}\right)$, xanthine $\left(50 \mu \mathrm{g} \mathrm{ml}^{-1}\right)$ or chloramphenicol $(21 \mu \mathrm{M})$ was performed according to the selection cassette used.

\section{Preparation of parasite genomic DNA}

Genomic DNA was extracted from $T$. gondii tachyzoites to use as a PCR template by pelleting parasites and resuspending in PBS. DNA extraction was then performed using the Qiagen QIAamp DNA blood mini kit as per the manufacturer's protocol.

\section{IFA}

Parasites were seeded onto HFFs grown on coverslips. $16-24 \mathrm{~h}$ after seeding, the coverslips were fixed in $3 \%$ formaldehyde for $15 \mathrm{~min}$ at room temperature then permeabilised in $0.2 \%$ Triton X-100/PBS for 3-10 min and blocked in 3\% BSA/PBS for $1 \mathrm{~h}$. Staining was performed using appropriate primary antibodies and goat Alexa Fluor 488-, Alexa Fluor 594- and Alexa Fluor 647-conjugated secondary antibodies (1:2000) alongside DAPI $(5 \mu \mathrm{g} / \mathrm{ml})$. Coverslips were mounted on glass slides with SlowFade gold antifade mountant (Life Technologies). Antibody concentrations used were: rat anti-HA high affinity (Roche, Basel, Switzerland) (1:1000), rabbit anti-TgCAP (1:2000), mouse anti-GFP (Roche) $(1: 1000)$.

Widefield images were generated with a Ti-E Nikon microscope using a $63 x$ or $100 x$ objective (Tokyo, Japan). Images were processed with Nikon Elements software. Confocal images were taken using a Zeiss LSM-780 inverted confocal laser scanning microscope with a $63 \mathrm{x}$ objective. Images were processed with Zeiss Zen Black software (Oberkochen, Germany). 


\section{Western blot}

Western blot samples were obtained by scraping and lysing intracellular parasites in $200 \mu \mathrm{l} 1 \mathrm{x}$ Laemmli buffer (2\% SDS, 10\% glycerol, 5\% 2-mercaptoethanol, 0.002\% bromophenol blue and $125 \mathrm{mM}$ Tris $\mathrm{HCl}, \mathrm{pH}$ 6.8). Samples were subjected to SDS-PAGE under reducing conditions before being transferred to a nitrocellulose membrane. Immunoblotting was performed with appropriate primary antibodies in $0.1 \%$ Tween $20,3 \%$ skimmed milk/PBS. Bound secondary fluorochrome-conjugated antibodies were visualised using the Odyssey Infrared Imaging System (LI-COR Biosciences, Nebraska, United States).

Antibody concentrations used were: rat anti-HA high affinity (Roche) (1:1000), rabbit antiTgCAP (1:2000), mouse anti-Toxoplasma [TP3] (Abcam, Cambridge, United Kingdom) (1:1000). Goat anti-mouse IRDye 800CW (LI-COR) (1:20000), Donkey anti-rabbit IRDye 680LT (LI-COR) (1:20000), Goat anti-rat IRDye 680LT (LI-COR) (1:20000).

\section{Generation of TgCAP antibody}

To generate the shortCAP expression plasmid, pET-28_CAP_A38toC203, a Toxoplasma shortCAP (M37toC203) recodonised sequence was synthesised (gBlock gene fragments, Integrated DNA Technologies) and Gibson cloned (Gibson et al., 2010) into BamHI and Ndel digested pET-28a(+) plasmid (Merck, Darmstadt, Germany). This allowed for expression of an $\mathrm{N}$-terminal 6xHis tagged shortCAP recombinant protein in Escherichia coli BL21 cells under the control of T7 lac promoter. Short CAP was expressed and His tag purified using Ni-NTA affinity purification under native conditions using the standard manufacturer's protocol (Qiagen, Hilden, Germany). The shortCAP recombinant protein was used to immunize female New Zealand white rabbits (Covalab, Cambridge, United Kingdom) for generation of polyclonal antibodies.

\section{Flow cytometry analysis of DiCre excision}

Parasites were added to a HFF monolayer and allowed to invade for $1 \mathrm{~h}$. Then, cre recombinase-mediated recombination was induced by addition of $50 \mathrm{nM}$ rapamycin in DMSO for $4 \mathrm{hr}$ before washout. $22 \mathrm{~h}$ after infection, parasites were lysed, pelleted and washed twice in PBS. Parasites were resuspended in $0.5 \mathrm{ml} 3 \%$ formaldehyde and fixed for $10 \mathrm{~min}$. The suspension was centrifuged and the pellet washed in PBS before resuspension in PBS. To remove debris, samples were passed through a $30 \mu \mathrm{m}$ pre-separation filter (Miltenyi Biotec, Bergisch Gladbach, Germany). 20,000 events were recorded using a BD LSR II flow cytometer (BD Biosciences California, United States). Killer Red was excited by the $561 \mathrm{~nm}$ laser and detected by a 600 long pass filter and either a $582 / 15,610 / 20$ or $620 / 40$ band pass filter. YFP was excited by the $488 \mathrm{~nm}$ laser and detected a 505 long pass filter and either a 525/50 or $530 / 30$ band pass filter. For quantification, and elimination of debris, total number of Killer Red+ parasites was considered $100 \%$. This was performed at day 0,35 and 65 of the experiment. For each condition, three biological replicates were analysed. At least 10000 Killer Red+ events were counted for each individual time point.

\section{Phenotypic characterisations Competition Assay}

mCherry-expressing $\triangle \mathrm{CAP}, \triangle \mathrm{CAP} \mathrm{PAP}^{\mathrm{C}}, \triangle \mathrm{CAP} \mathrm{P}^{\text {shortCAP }}$ or $\triangle \mathrm{CAP} \mathrm{P}^{\text {longCAP }}$ parasites were mixed with non-fluorescent $\triangle C A P^{C A P}$ parasites at an average ratio of $60 / 40$. At day 0,15 and 30 in culture, the ratio was determined by flow cytometry for two biological replicates. Parasites were lysed, pelleted and washed twice in PBS. Parasites were resuspended in $0.5 \mathrm{ml} 3 \%$ formaldehyde and fixed for $5 \mathrm{~min}$. The suspension was centrifuged and the pellet resuspended in $5 \mu \mathrm{g} / \mathrm{ml}$ DAPI/PBS for $10 \mathrm{~min}$. The pellet was washed and resuspended in PBS. To remove debris, samples were passed through a $30 \mu \mathrm{m}$ pre-separation filter (Miltenyi Biotec). Events were recorded using a BD LSR II flow cytometer (BD Biosciences). DAPI was excited by the 355 $\mathrm{nm}$ laser and detected by a 450/50 band pass filter. mCherry was excited by the $561 \mathrm{~nm}$ laser and detected by a 600 long pass filter and a $610 / 20$ band pass filter. To eliminate debris from 
the analysis, events were gated on DAPI fluorescence. The ratio of control parasites (DAPI+/mCherry-) to individual CAP complements (DAPI+/mCherry+) was calculated and normalised to the day 0 ratio. The data represent two (day 15) and three (day 30) independent experiments. At least 1500 DAPI+ events were obtained for each individual time point. The results were statistically tested with a two-way ANOVA test plus a multiple comparison Sidak's test individually comparing day 15 or day 30 means to their respective day 0 mean, in GraphPad Prism® 7. The data presented are as mean \pm s.d.

\section{Intracellular growth and rosetting assay}

Parasites were harvested from a T-25 and added to a coverslip coated with a HFF monolayer. After $20 \mathrm{~h}$ the coverslips were fixed with $3 \%$ formaldehyde for $15 \mathrm{~min}$ at room temperature. Coverslips were mounted and mCherry expression used to identify parasites. For each replicate, 4 random fields were imaged with a 40x objective. Counts were performed in a blinded manner in duplicate for two independent experiments. The number of parasites per vacuole was determined by counting at least 265 vacuoles per strain. The number of vacuoles that rosette was determined by looking at 8-pac vacuoles, at least 90 vacuoles per strain were counted. The results were statistically tested with a one-way ANOVA test plus a multiple comparison Dunnett's test comparing all means to the $\triangle$ CAP $^{\mathrm{CAP}}$ mean in GraphPad Prism ${ }^{\circledR} 7$. The data presented are as mean \pm s.d.

\section{Invasion assay}

Red/green invasion assays were performed. mCherry-expressing parasites were lysed in an invasion non-permissive buffer, Endo buffer (44.7 $\mathrm{mM} \mathrm{K}_{2} \mathrm{SO}_{4}, 10 \mathrm{mM} \mathrm{MgSO} 4,106 \mathrm{mM}$ sucrose, $5 \mathrm{mM}$ glucose, $20 \mathrm{mM}$ Tris- $\mathrm{H}_{2} \mathrm{SO}_{4}, 3.5 \mathrm{mg} / \mathrm{ml} \mathrm{BSA}, \mathrm{pH} 8.2$ ). $250 \mu \mathrm{l}$ of $8 \times 10^{5}$ parasites $/ \mathrm{ml}$ in Endo buffer were added to each well of a 24-well flat-bottom plate [Falcon], which contains a coverslip with a confluent HFF monolayer. The plates were spun at $129 \times \mathrm{g}$ for $1 \mathrm{~min}$ at $37^{\circ} \mathrm{C}$ to deposit parasites onto the monolayer. The Endo buffer was gently removed and replaced with invasion permissive medium (1\% FBS/DMEM). These parasites were allowed to invade for $1 \mathrm{~min}$ at $37^{\circ} \mathrm{C}$ after which the monolayer was gently washed twice with PBS and fixed with $3 \%$ formaldehyde for $15 \mathrm{~min}$ at room temperature. Extracellular parasites were stained with mouse anti-Toxoplasma antigen B1247M (Abcam) 1:1000 and goat antimouse Alexa Fluor 488, following the IFA protocol. For each replicate, 3 random fields were imaged with a 40x objective. Three independent experiments were performed in duplicate. The number of intracellular (mCherry+/488-) and extracellular (mCherry+/488+) parasites was determined by counting, in a blinded fashion, at least 758 parasites per strain. The results were statistically tested with a one-way ANOVA test plus a multiple comparison Dunnett's test

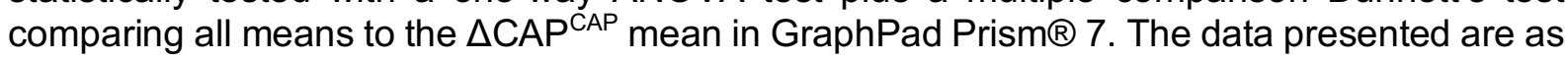
mean \pm s.d.

\section{Egress assay}

Parasites were added to a HFF monolayer, in a ibidi $\mu$-plate 96 well, and grown for $30 \mathrm{~h}$. The wells were washed twice with PBS and the media was exchanged for $80 \mu$ Ringers solution (155 mM NaCl, $3 \mathrm{mM} \mathrm{KCl}, 2 \mathrm{mM} \mathrm{CaCl}, 1 \mathrm{mM} \mathrm{MgCl}_{2}, 3 \mathrm{mM} \mathrm{NaH}_{2} \mathrm{PO}_{4}, 10 \mathrm{mM}$ HEPES, 10 $\mathrm{mM}$ glucose). To artificially induce egress, $40 \mu \mathrm{l}$ of Ringer's solution containing $150 \mu \mathrm{M}$ BIPPO (50 $\mu \mathrm{M}$ final conc) was added to each well. At specified time points the cells were fixed by adding $26 \mu \mathrm{l} 16 \%$ formaldehyde (3\% final conc) for $15 \mathrm{~min}$. Cells were washed in PBS and stained with DAPI $(5 \mu \mathrm{g} / \mathrm{ml})$. Automated image acquisition of 25 fields per well was performed on a Cellomics Array Scan VTI HCS reader (Thermo Scientific, Massachusetts, United States) using a 20x objective. Image analysis was performed using the Compartmental Analysis BioApplication on HCS Studio (Thermo Scientific). Egress levels were determined in triplicate for three independent assays. At least 11987 vacuoles per strain were counted at $t=0 \mathrm{~s}$. Subsequent time point vacuole counts were normalised to $t=0$ to determine how many vacuoles had egressed. The results were statistically tested with a two-way ANOVA test plus 
a multiple comparison Dunnett's test comparing all means to the $\triangle \mathrm{CAP}^{\mathrm{CAP}}$ mean, at each time point separately, in GraphPad Prism ${ }^{\circledR} 7$. The data presented are as mean \pm s.d.

\section{Live egress}

881

Parasites were added to a HFF monolayer, in a ibidi $\mu$-plate 96 well, and grown for $30 \mathrm{~h}$. The wells were washed twice with PBS and the media was exchanged for $80 \mu$ l Ringers solution $\left(155 \mathrm{mM} \mathrm{NaCl}, 3 \mathrm{mM} \mathrm{KCl}, 2 \mathrm{mM} \mathrm{CaCl}_{2}, 1 \mathrm{mM} \mathrm{MgCl}\right.$, $3 \mathrm{mM} \mathrm{NaH}_{2} \mathrm{PO}_{4}, 10 \mathrm{mM}$ HEPES, 10 $\mathrm{mM}$ glucose). The plate was then transferred to a Ti-E Nikon microscope with a $37^{\circ} \mathrm{C}$ environmental chamber. To artificially induce egress, $40 \mu$ l of Ringer's solution containing 150 $\mu \mathrm{M}$ BIPPO (50 $\mu \mathrm{M}$ final conc) was added to each well after imaging had commenced. Images were captured every $1.8 \mathrm{~s}$.

\section{Apicoplast segregation assay}

Parasites were added to a HFF monolayer and grown for $20 \mathrm{~h}$ before fixation with ice cold methanol for $2 \mathrm{~min}$ at room temperature. IFAs were performed using a streptavidin-Alexa Fluor 594 conjugate (Invitrogen) as a marker for the apicoplast. Correct apicoplast segregation was determined in duplicate for three independent assays. At least 228 vacuoles were counted per strain. Counts were performed in a blinded manner. The results were statistically tested with an unpaired t test in GraphPad Prism® ${ }^{\circledR}$. The data presented are as mean \pm s.d.

\section{Synchronicity of division and daughter cell orientation assay}

Parasites were added to a HFF monolayer and grown for $20 \mathrm{~h}$ before fixation with $3 \%$ formaldehyde for $15 \mathrm{~min}$ at room temperature. IFAs were performed using rat anti-IMC3 antibodies. To determine the synchronicity of cell division within the vacuoles, anti-IMC3 staining was used to evaluate the stage of daughter cell development. Vacuoles were scored as synchronous if all daughter cells were at the same stage of development. Vacuoles were counted blind in triplicate for three independent experiments. At least 275 vacuoles were counted per strain. The results were statistically tested with an unpaired $t$ test in GraphPad Prism ${ }^{\circledR} 7$. The data presented are as mean \pm s.d. Daughter cell orientation was quantified in triplicate for three independent experiments. At least 312 mother cells were counted blind, per strain. The results were statistically tested with a one-way ANOVA test plus a multiple comparison Dunnett's test comparing all means to the $\triangle \mathrm{CAP}^{\mathrm{CAP}}$ mean in GraphPad Prism ${ }^{\circledR} 7$. The data presented are as mean \pm s.d.

\section{D motility assay}

Motility assays were performed as previously described (Leung et al., 2014), with minor modifications. Parasites were syringe-released from a HFF monolayer (one heavily infected T75 flask per strain) by passing through a 27 gauge needle and filtering through a $3 \mu \mathrm{m}$ Nuclepore filter. Parasites were then centrifuged (1,000 x g for $2 \mathrm{~min}$ ) and resuspended in 40 $\mu \mathrm{l}$ motility media supplemented with $0.3 \mathrm{mg} / \mathrm{mL}$ Hoescht 33342 . Matrigel was thawed on ice to prevent polymerization and combined with parasites and motility media in a ratio of 3:1:3 respectively. Pitta chambers were perfused with $10 \mu \mathrm{L}$ of this suspension and incubated at $27^{\circ} \mathrm{C}$ for 7 minutes on a thermoplate. Chambers were incubated in the heated $\left(35^{\circ} \mathrm{C}+/-1^{\circ} \mathrm{C}\right)$ microscope enclosure for 3 min prior to imaging. Parasite nuclei were imaged, using a $20 \mathrm{x}$ objective, capturing $61-63$ stacks of 41 z-slices $1 \mu \mathrm{m}$ apart. To ensure conditions remained constant between parasite lines, samples used for capture were alternated. Datasets were exported to Imaris $\times 64$ v9.2.1. Using the ImarisTrack module parasites were tracked in a region of interest from which $1 \mu \mathrm{m}$ has been cropped from the $\mathrm{x}$ and $\mathrm{y}$ edges to eliminate edge artefacts. Parasites were identified, after background subtraction, using spot detection with estimated size of $4 \mu \mathrm{m}(\mathrm{xy})$ and $8 \mu \mathrm{m}(\mathrm{z})$. Spots were filtered to exclude all that had duration of less than 3 seconds to minimise tracking artifacts. An autoregressive motion tracking algorithm was applied with a maximum distance of $6 \mu \mathrm{m}$ and a maximum gap size of 3 . Datasets were manually inspected to ensure appropriate tracking and to remove artifacts and trajectories tracked from multiple identified spots. Percent moving was calculated as the 
number of trajectories ( $>2 \mu \mathrm{m}$ displacement) / number of objects in frame 3 ( $>3 \mathrm{~s}$ duration). Trajectory parameters were extracted directly from Imaris software. Data shown are derived from 4 independent biological replicates, each consisting of a minimum of two technical replicates. For all analysis, means and standard deviation were calculated for four independent biological replicates before statistical analysis using an unpaired t-test in GraphPad Prism $\circledR 7$. For the three strain experiment, $\triangle \mathrm{CAP} \mathrm{P}^{\text {shortCAP }}$ and $\triangle \mathrm{CAP} \mathrm{P}^{\text {longAP }}$ were each compared to $\triangle \mathrm{CAP}^{\mathrm{CAP}}$. All technical replicates are presented in the figures, together with their mean $\pm \mathrm{s} . \mathrm{d}$.

\section{Dense granule trafficking assay}

For each condition $1 \times 10^{7}$ parasites were transiently transfected with $30 \mu \mathrm{g}$ of pTub SAG $1 \triangle \mathrm{GPI}$ mCherry plasmid (Heaslip et al., 2016) and immediately added to a confluent HFF monolayer in a Mattek $B 35 \mathrm{~mm}$ dish, coverslip 1.5. 12-15 h after infection the monolayers were washed 3 times with pre-warmed Gibco® Fluorobrite ${ }^{\mathrm{TM}}$ DMEM supplemented with $4 \%$ Fetal Bovine Serum. The coverslips were immediately used for imaging. The acquisitions were made with an Olympus IX71 coupled to a DELTAVISION ${ }^{\mathrm{TM}}$ Elite imaging system in a $37^{\circ} \mathrm{C}$ environmental chamber. The acquisition for each condition were made sequentially from the washing step to the acquisition with a random order to avoid any artifactual data. The acquisition analysis was made with Fiji and the MTrackJ plugin. The data represent three independent experiments. At least 43 parasites and 273 direct runs were counted per strain. The results were statistically tested with a one-way ANOVA test plus a multiple comparison Dunnett's test comparing all means to the $\triangle \mathrm{CAP}^{\mathrm{CAP}}$ mean in GraphPad Prism ${ }^{\circledR} 7$. The data presented are as mean \pm s.d.

\section{FRAP}

Parasites were inoculated on a confluent layer of HFFs $20 \mathrm{~h}$ before experiments were performed using a Zeiss LSM-780 inverted confocal laser scanning microscope at $37^{\circ} \mathrm{C}$. Acquisition and processing were performed with the Zeiss Zen Black software. Images were taken for 2 min (one image per second). Three pre-bleach images were recorded before the region of interest was photobleached ten times with a $561 \mathrm{~nm}$ laser at $100 \%$ power. Fluorescence intensity is presented as a percentage relative to the same area pre-bleach. These normalised intensity values were also used for the calculations below. To calculate percentage of fluorescence recovery, the final reading (116 s post-bleach) was subtracted from the reading immediately post-bleach, $t=3 \mathrm{~s}$, to give percentage recovery after $116 \mathrm{~s}$. To calculate percentage loss of fluorescence, the reading immediately post-bleach, $t=3 \mathrm{~s}$, was subtracted from the final reading (116 s post-bleach) to give percentage loss after $116 \mathrm{~s}$. These percentages were used to generate the heat map. At least 11 vacuoles were counted per strain across at least two independent experiments. The results were statistically tested with a oneway ANOVA test plus a multiple comparison Dunnett's test comparing all means to the $\triangle$ CAP $^{\mathrm{CAP}}$ mean in GraphPad Prism ${ }^{\circledR} 7$. The data presented are as mean \pm s.d. To assess recovery type, at least 11 vacuoles were counted per strain across at least two independent experiments. The results were statistically tested with a Chi-square in GraphPad Prism ${ }^{\circledR} 7$.

\section{Tape unroofing SEM}

Parasites were inoculated on a confluent HFF monolayer $24 \mathrm{~h}$ before fixation in EM fixative (2.5\% gluteraldehyde, $4 \%$ formaldehyde in $0.1 \mathrm{M}$ phosphate buffer) for $30 \mathrm{~min}$. Cells were washed in $0.1 \mathrm{M}$ phosphate buffer (PB) and stored in $1 \%$ formaldehyde in PB at $4^{\circ} \mathrm{C}$. Cells were then washed in $\mathrm{PB}$ at room temperature, then washed in $\mathrm{dd}_{2} \mathrm{O}$ at $\mathrm{RT}$. The cells were dehydrated stepwise from $70 \%$ to $100 \%$ ethanol before critical point drying from acetone in a CPD300 (Leica Microsystems, Vienna, Austria). After drying, the coverslips were mounted on stubs, and the HFF cells were unroofed by placing Scotch tape on the coverslips and gently peeling it off, exposing the host cytoplasm and the parasitophorous vacuoles. The cells were coated with $7 \mathrm{~nm}$ platinum in a Q150R Sputter Coater (Quorum Tech, East Sussex, UK) before viewing in a Phenom ProX SEM (Thermo Scientific) at $10 \mathrm{kV}, 1024 \times 1024$ pixel frame, on 'high' quality. 


\section{FIB SEM}

Immediately following FRAP experimentation, as described above, parasites were fixed in 4\% formaldehyde for $15 \mathrm{~min}$ at $37^{\circ} \mathrm{C}$ before washing in $0.1 \mathrm{M} \mathrm{PB}$. Cells were then fixed in EM fixative $(2.5 \%$ gluteraldehyde, $4 \%$ formaldehyde in $0.1 \mathrm{M} \mathrm{PB})$ for $30 \mathrm{~min}$ at room temperature. Cells were washed in $0.1 \mathrm{M} \mathrm{PB}$ and stored in $1 \%$ formaldehyde in $0.1 \mathrm{M} \mathrm{PB}$. After fixation, samples were transferred to a Pelco BioWave Pro+ microwave (Ted Pella) for processing using a protocol adapted from the NCMIR protocol (Deerinck et al., 2010). See Supplementary File 3 for full BioWave program details. The SteadyTemp plate was set to $21^{\circ} \mathrm{C}$ unless otherwise indicated. Each step was performed in the microwave, except for the buffer and $\mathrm{ddH}_{2} \mathrm{O}$ wash steps, which consisted of two washes on the bench and two washes in the microwave ( $250 \mathrm{~W}$ for $40 \mathrm{~s}$ ). The cells were washed (as above) in $0.1 \mathrm{M} \mathrm{PB}$, stained with $2 \%$ osmium tetroxide and $1.5 \%$ potassium ferricyanide $(\mathrm{v} / \mathrm{v})$ for $14 \mathrm{~min}$ under vacuum (with/without $100 \mathrm{~W}$ power at 2 min intervals), and then washed in $\mathrm{ddH}_{2} \mathrm{O}$ (as above). Next, the cells were incubated in $1 \%$ thiocarbohydrazide in $\mathrm{ddH}_{2} \mathrm{O}(\mathrm{w} / \mathrm{v})$ for $14 \mathrm{~min}$ (vacuum, $100 \mathrm{~W}$ on/off at 2 min intervals) with SteadyTemp plate set to $40^{\circ} \mathrm{C}$, followed by $\mathrm{dd}_{2} \mathrm{O}$ washes (as above), and then a further stain with $2 \%$ osmium tetroxide in $\mathrm{ddH}_{2} \mathrm{O}(\mathrm{w} / \mathrm{v})$ for $14 \mathrm{~min}$ (vacuum, $100 \mathrm{~W}$ on/off at 2 min intervals), followed by $\mathrm{ddH}_{2} \mathrm{O}$ washes (as above). The cells were then incubated in $1 \%$ aqueous uranyl acetate (vacuum, $100 \mathrm{~W}$ on/off at 2 min intervals, SteadyTemp $40^{\circ} \mathrm{C}$ ), and then washed in $\mathrm{ddH}_{2} \mathrm{O}$ (as above, except with SteadyTemp at $40^{\circ} \mathrm{C}$ ). Walton's lead aspartate was then applied (vacuum, $100 \mathrm{~W}$ on/off at $2 \mathrm{~min}$ intervals, SteadyTemp $50^{\circ} \mathrm{C}$ ), and the cells were washed (as above) and dehydrated in a graded ethanol series $(70 \%, 90 \%$, and $100 \%$, twice each), at $250 \mathrm{~W}$ for $40 \mathrm{~s}$ without vacuum. Exchange into Durcupan $A C M \otimes$ resin (Sigma-Aldrich, Missouri, United States) was performed in $50 \%$ resin in ethanol, at $250 \mathrm{~W}$ for 3 min, with vacuum cycling (on/off at $30 \mathrm{sec}$ intervals), and then pure Durcupan was infiltrated in four microwave steps with the same settings, before embedding at $60^{\circ} \mathrm{C}$ for $48 \mathrm{~h}$.

Focused ion beam scanning electron microscopy (FIB SEM) data was collected using a Crossbeam 540 FIB SEM with Atlas 5 for 3-dimensional tomography acquisition (Zeiss). Segments of the cell monolayer containing the cells of interest were trimmed, polished with a diamond knife (removing uneven resin at the base of the monolayer to provide a flat surface for tracking marks), mounted on a standard $12.7 \mathrm{~mm}$ SEM stub using conductive epoxy (ITW Chemtronics), and coated with a $5 \mathrm{~nm}$ layer of platinum.

The specific cells of interest were relocated by imaging through the platinum coating at an accelerating voltage of $20 \mathrm{kV}$ and correlating to previously acquired fluorescence microscopy images. After preparation for milling and tracking, images were acquired at $5 \mathrm{~nm}$ isotropic resolution throughout each region of interest, using a $10 \mu \mathrm{s}$ dwell time. During acquisition the SEM was operated at an accelerating voltage of $1.5 \mathrm{kV}$ with $1 \mathrm{nA}$ current. The EsB detector was used with a grid voltage of $1,200 \mathrm{~V}$. Ion beam milling was performed at an accelerating voltage of $30 \mathrm{kV}$ and current of $700 \mathrm{pA}$.

After cropping to the specific region of interest comprising the entire extent of the PV ( $\triangle \mathrm{CAP} \mathrm{PAP}^{\mathrm{C}}$; $3080 \times 634 \times 2509$ pixels; $15.4 \times 3.17 \times 12.545 \mu \mathrm{m} ; \Delta \mathrm{CAP} ; 4065 \times 1136 \times 4490$ pixels; 20.325 $\times 5.68 \times 22.45 \mu \mathrm{m}$ ) and aligning the dataset (gradient align; Atlas 5), the images were processed to supress noise and slightly enhance sharpness (gaussian blur 0.75 radius, followed by unsharp mask radius 1 , strength 0.6 ; Fiji) prior to reorienting and reslicing in the $\mathrm{YZ}$ plane (assigned as the $\mathrm{XY}$ plane for segmentation), and scaling to $10 \mathrm{~nm}$ isotropic resolution for segmentation and display.

Selected structures were segmented manually from the FIB SEM datasets and 3D reconstructions were made using the $3 \mathrm{dmod}$ program of IMOD (Kremer et al., 1996). The normal and decentralised residual body structures were manually skeletonised by tracing the approximate central axis of the structure from the posterior pore of each tachyzoite using open contours. Thus, the green skeleton follows the lumen of the connections between parasites. 
The minimum number of points were placed that would still ensure the contour remained at the central axis of the volume. Where the structure branched, points linking contours from multiple extensions were placed at the approximate centre of the branch point volume. The contours were then rendered as a 50 or $100 \mathrm{~nm}$ tube to aid visualisation (tube diameter chosen depending on view). This model was then inspected in the $\mathrm{X}, \mathrm{Y}$, and Z-planes and corrections made to ensure the skeleton followed the approximate centre through the volume. Since not all RB-like structure extensions ended at the posterior pole of a tachyzoite, the posterior poles were highlighted by segmenting them with open contours with points at every $40 \mathrm{~nm}$ in Z, following the edge of the cytosol (the ribosome-containing electron lucent space where it meets the intermediate electron density surrounding the inner membrane complex), and meshing the contour as a $50 \mathrm{~nm}$ tube. A selected region of the putative ER lipid bilayer outer leaflet was segmented with closed contours drawn every 5-20 nm in Z (smaller Z intervals where needed to capture fenestrations/complexity); from an approximately square region around the inner face of two of the basal pores up to an arbitrary point along the decentralised residual body structure. Contour gaps were placed at the edge of this region. Two tachyzoites were also highlighted by partial coarse segmentation; closed contours drawn every $250 \mathrm{~nm}$ in Z through the main body of the cell (segmentation of the complex top and bottom of the tachyzoites was omitted for clarity).

\section{Animals}

1058

1059

C57BL/6 (wild type) mice were bred and housed under pathogen-free conditions in the biological research facility at the Francis Crick Institute in accordance with the Home Office UK Animals (Scientific Procedures) Act 1986. All work was approved by the UK Home Office (project license PDE274B7D), the Francis Crick Institute Ethical Review Panel, and conforms to European Union directive 2010/63/EU. All mice used in this study were male and between 7- to 9-week old.

1066

Mice were infected with $T$. gondii tachyzoites by intraperitoneal injection (i.p.) with either 25, 5 $\times 10^{3}$ parasites (cyst formation) or $5 \times 10^{4}$ parasites (survival) in $200 \mu \mathrm{l}$ medium on day 0 . Mice were monitored and weighed regularly for the duration of the experiments.

1068 For serum samples, mice were euthanized and blood collected into blood serum collection tubes (SAI, Infusion technologies) by puncturing the jugular vein. Blood was allowed to clot at room temperature for $30 \mathrm{~min}$, before tubes were centrifuged at $1500 \mathrm{x} \mathrm{g}$ for $10 \mathrm{~min}$. Serum was collected and stored at $-20^{\circ} \mathrm{C}$ until analysis.

\section{Toxoplasma serum antibody ELISA}

Toxoplasma soluble antigens were extracted as previously described (Silva et al., 2007). In short, parasites were syringe-lysed, washed once with PBS, and adjusted to $1 \times 10^{8}$ tachyzoites $/ \mathrm{ml}$ with PBS containing protease inhibitors (cOmplete mini, Roche). Parasites were lysed by five freeze-thaw cycles (liquid nitrogen $/ 37^{\circ} \mathrm{C}$ ), followed by ultrasound sonication on ice (five $60 \mathrm{~Hz}$ cycle for 1 min each). Samples were centrifuged at 10,000 x g for $30 \mathrm{~min}$ at $4{ }^{\circ} \mathrm{C}$ before supernatants were collected and protein content was determined using the BCA Protein Assay Kit (Pierce, Thermo Fisher Scientific) following the manufacturer's instructions.

To detect Toxoplasma antibodies in murine serum samples, 96-well plates (flat bottom, highbinding) were coated overnight with $2 \mu \mathrm{g} / \mathrm{ml}$ Toxoplasma soluble antigens at $4^{\circ} \mathrm{C}$. Plates were washed with PBS/0.05\% Tween-20 (v/v) (PBS-T) before blocked with 1\% BSA (w/v) in PBS for $2 \mathrm{~h}$ at room temperature. Bound antigens were incubated with murine sera diluted 1/10 in $1 \%$ BSA/PBS for $2 \mathrm{~h}$ at room temperature, washed three times with PBS-T and bound antibodies detected by incubation for $2 \mathrm{~h}$ at room temperature with anti-mouse Immunoglobulins (HRP conjugate, Darko) diluted 1/1000 in 1\% BSA/PBS. Finally, plates were washed three times with PBS-T and developed by adding TMB substrate solution (Thermo Fisher Scientific). The TMB reaction was stopped by adding $2 \mathrm{~N}$ sulphuric acid and the absorbance measured $\left(\mathrm{OD}_{450}\right.$ minus $\mathrm{OD}_{540}$ wave length correction) using the VersaMax ${ }^{\mathrm{TM}}$ Microplate Reader with SoftMax® Pro Software. 


\section{Mouse brain collection and preparation for cyst counting}

To determine the number of cysts in the brain of infected animals, mice were euthanized and the brain extracted from the skull. The brain was homogenised in $1 \mathrm{ml}$ PBS and stained with Rhodamine-conjugated Dolichos biflorus agglutinin (1/1000; Vector Laboratories) for $1 \mathrm{~h}$ at room temperature. Fluorescently labelled cysts were counted using a Ti-E Nikon microscope.

\section{Statistical analysis}

Statistical tests used are stated in individual sections above. $P$-values significance thresholds were set at: ${ }^{* * *} P<0.0001,{ }^{* * *} P<0.001,{ }^{* *} P<0.01$ and ${ }^{*} P<0.05$. All significant results are labelled with a line and asterisk(s) in the graphs.

Acknowledgements

We thank Marc-Jan Gubbels (Boston College) for gifting the IMC3 antibody, Markus Meissner (University of Glasgow) for the pG140 plasmid, Michael Reese (University of Texas Southwestern Medical Center) for the pUPRT-HA plasmid, David Sibley (Washington University) for the CRISPR/Cas9 plasmid and Caia Dominicus (Francis Crick Institute) for critically reading the manuscript. We also thank the following members of science technology platforms at the Francis Crick Institute for their support: Matt Renshaw (light microscopy), Michael Howell (high throughput screening), Damian Carragher, Rhys Hefin, Phil Hobson and Graham Preece (flow cytometry). This work was supported by awards to MT by The Francis Crick Institute (https://www.crick.ac.uk/), which receives its core funding from Cancer Research UK (FC001189; https://www.cancerresearchuk.org), the UK Medical Research Council (FC001189; https://www.mrc.ac.uk/) and the Wellcome Trust (FC001189; https://wellcome.ac.uk/). This work was supported by US Public Health Service grants Al137767 and Al139201 to GEW and National Institutes of Health grant awarded to Aoife Heaslip (Al121885).

\section{Competing interests}

The authors have declared that no competing interests exist.

\section{References}

Andenmatten, N., Egarter, S., Jackson, A. J., Jullien, N., Herman, J.-P., \& Meissner, M. (2013). Conditional genome engineering in Toxoplasma gondii uncovers alternative invasion mechanisms. Nature Methods, 10(2), 125-127. doi:10.1038/nmeth.2301

1129 Bannister, L. H., \& Mitchell, G. H. (1995). The role of the cytoskeleton in Plasmodium falciparum merozoite biology: an electron-microscopic view. Annals of Tropical Medicine and Parasitology, 89(2), 105-111. doi:10.1080/00034983.1995.11812940

1132 Baum, J., Papenfuss, A. T., Baum, B., Speed, T. P., \& Cowman, A. F. (2006). Regulation of apicomplexan actin-based motility. Nature Reviews Microbiology, 4(8), 621-628. 
1135

1136

1137

1138

1139

1140

1141

1142

1143

1144

1145

1146

1147

1148

1149

1150

1151

1152

1153

1154

1155

1156

1157

1158

Bertling, E., Hotulainen, P., Mattila, P. K., Matilainen, T., Salminen, M., \& Lappalainen, P. (2004). Cyclase-associated protein 1 (CAP1) promotes cofilin-induced actin dynamics in mammalian nonmuscle cells. Molecular Biology of the Cell, 15(5), 2324-2334. doi:10.1091/mbc.e04-01-0048

Black, M. W., Arrizabalaga, G., \& Boothroyd, J. C. (2000). lonophore-resistant mutants of Toxoplasma gondii reveal host cell permeabilization as an early event in egress. Mol Cell Biol, 20(24), 9399-408. doi:10.1128/MCB.20.24.9399-9408.2000

Black, M. W., \& Boothroyd, J. C. (2000). Lytic cycle of Toxoplasma gondii. Microbiology and Molecular Biology Reviews, 64(3), 607-623. doi:10.1128/MMBR.64.3.607-623.2000

Brown, K. M., Long, S., \& Sibley, L. D. (2017). Plasma membrane association by N-acylation governs PKG function in Toxoplasma gondii. MBio, 8(3), e00375-17.

Caffaro, C. E., Koshy, A. A., Liu, L., Zeiner, G. M., Hirschberg, C. B., \& Boothroyd, J. C. (2013). A nucleotide sugar transporter involved in glycosylation of the Toxoplasma tissue cyst wall is required for efficient persistence of bradyzoites. PLOS Pathogens, 9(5), e1003331. doi:10.1371/journal.ppat.1003331

Deerinck, T. J., Bushong, E. A., Thor, A., \& Ellisman, M. H. (2010). NCMIR methods for 3D EM: A new protocol for preparation of biological specimens for serial block face scanning electron microscopy. Retrieved November 21, 2018, from http://gatan.actonservice.com/acton/attachment/11413/f-017e/1/-/-/-//sbfsem\%20sample\%20prep\%20protocol.pdf?modal=1

Delbac, F., Sänger, A., Neuhaus, E. M., Stratmann, R., Ajioka, J. W., Toursel, C., Herm-Götz, A., Tomavo, S., Soldati, T., \& Soldati, D. (2001). Toxoplasma gondii myosins B/C: one gene, two tails, two localizations, and a role in parasite division. The Journal of Cell Biology, 155(4), 613-624. doi:10.1083/jcb.200012116 
1159

1160

1161

1162

1163

1164

1165

1166

1167

1168

1169

1170

1171

1172

1173

1174

1175

1176

1177

1178

1179

1180

1181

1182

Dobrowolski, J. M., Niesman, I. R., \& Sibley, L. D. (1997). Actin in the parasite Toxoplasma gondii is encoded by a single copy gene, ACT1 and exists primarily in a globular form. Cell Motility, 37(3), 253-262. doi:10.1002/(SICI)1097-0169(1997)37:3<253::AID$\mathrm{CM} 7>3.0 . \mathrm{CO} ; 2-7$

Egarter, S., Andenmatten, N., Jackson, A. J., Whitelaw, J. A., Pall, G., Black, J. A., Ferguson, D. J. P., Tardieux, I., Mogilner, A., \& Meissner, M. (2014). The Toxoplasma acto-MyoA motor complex is important but not essential for gliding motility and host cell invasion. PLoS ONE, 9(3), e91819. doi:10.1371/journal.pone.0091819

Foe, I. T., Child, M. A., Majmudar, J. D., Krishnamurthy, S., van der Linden, W. A., Ward, G. E., Martin, B. R., \& Bogyo, M. (2015). Global analysis of palmitoylated proteins in Toxoplasma gondii. Cell Host \& Microbe, 18(4), 501-511. doi:10.1016/j.chom.2015.09.006

Foe, I. T., Onguka, O., Amberg-Johnson, K., Garner, R. M., Amara, N., Beatty, W., Yeh, E., \& Bogyo, M. (2018). The Toxoplasma gondii active serine hydrolase 4 regulates parasite division and intravacuolar parasite architecture. MSphere, 3(5), e00393-18. doi:10.1128/mSphere.00393-18

Fox, B. A., Ristuccia, J. G., Gigley, J. P., \& Bzik, D. J. (2009). Efficient gene replacements in Toxoplasma gondii strains deficient for nonhomologous end joining. Eukaryotic Cell, 8(4), 520-529. doi:10.1128/EC.00357-08

Frénal, K., Dubremetz, J.-F., Lebrun, M., \& Soldati-Favre, D. (2017). Gliding motility powers invasion and egress in Apicomplexa. Nature Reviews Microbiology, 15, 645. doi:10.1038/nrmicro.2017.86

Frénal, K., Jacot, D., Hammoudi, P.-M., Graindorge, A., Maco, B., \& Soldati-Favre, D. (2017). Myosin-dependent cell-cell communication controls synchronicity of division in acute 
and chronic stages of Toxoplasma gondii. Nature Communications, 8, ncomms15710. doi:10.1038/ncomms15710

1185

1186

1187

1188

1189

1190

1191

1192

1193

1194

1195

1196

1197

1198

1199

1200

1201

1202

1203

1204

1205

Garrison, E., Treeck, M., Ehret, E., Butz, H., Garbuz, T., Oswald, B. P., Settles, M., Boothroyd, J., \& Arrizabalaga, G. (2012). A forward genetic screen reveals that calciumdependent protein kinase 3 regulates egress in Toxoplasma. PLoS Pathog, 8(11). doi:10.1371/journal.ppat.1003049

Gibson, D. G., Smith, H. O., lii, C. A. H., Venter, J. C., \& Merryman, C. (2010). Chemical synthesis of the mouse mitochondrial genome. Nature Methods, 7(11), 901-903. doi:10.1038/nmeth.1515

Graindorge, A., Frénal, K., Jacot, D., Salamun, J., Marq, J. B., \& Soldati-Favre, D. (2016). The conoid associated motor $\mathrm{MyoH}$ is indispensable for Toxoplasma gondii entry and exit from host cells. PLoS Pathog, 12(1), e1005388. doi:10.1371/journal.ppat.1005388

Haase, S., Zimmermann, D., Olshina, M. A., Wilkinson, M., Fisher, F., Tan, Y. H., Stewart, R. J., Tonkin, C. J., Wong, W., Kovar, D. R., \& others. (2015). Disassembly activity of actindepolymerizing factor (ADF) is associated with distinct cellular processes in apicomplexan parasites. Molecular Biology of the Cell, 26(17), 3001-3012. doi:10.1091/mbc.E14-10-1427

Hakimi, M.-A., Olias, P., \& Sibley, L. D. (2017). Toxoplasma effectors targeting host signaling and transcription. Clinical Microbiology Reviews, 30(3), 615-645. doi:10.1128/CMR.00005-17

Halonen, S. K., \& Weiss, L. M. (2013). Chapter 8 - Toxoplasmosis. In H. H. Garcia, H. B. Tanowitz, \& O. H. Del Brutto (Eds.), Handbook of Clinical Neurology (Vol. 114, pp. 125-145). Elsevier. doi:10.1016/B978-0-444-53490-3.00008-X 
1206

1207

1208

1209

1210

1211

1212

1213

1214

1215

1216

1217

1218

1219

1220

1221

1222

1223

1224

1225

1226

1227

1228

Heaslip, A. T., Nelson, S. R., \& Warshaw, D. M. (2016). Dense granule trafficking in Toxoplasma gondii requires a unique class 27 myosin and actin filaments. Molecular Biology of the Cell, 27(13), 2080-2089. doi:10.1091/mbc.E15-12-0824

Hliscs, M., Sattler, J. M., Tempel, W., Artz, J. D., Dong, A., Hui, R., Matuschewski, K., \& Schuler, H. (2010). Structure and function of a G-actin sequestering protein with a vital role in malaria oocyst development inside the mosquito vector. Journal of Biological Chemistry, 285(15), 11572-11583. doi:10.1074/jbc.M109.054916

Howard, B. L., Harvey, K. L., Stewart, R. J., Azevedo, M. F., Crabb, B. S., Jennings, I. G., Sanders, P. R., Manallack, D. T., Thompson, P. E., Tonkin, C. J., \& Gilson, P. R. (2015). Identification of potent phosphodiesterase inhibitors that demonstrate cyclic nucleotide-dependent functions in apicomplexan parasites. ACS Chemical Biology, 10(4), 1145-1154. doi:10.1021/cb501004q

Huynh, M.-H., \& Carruthers, V. B. (2009). Tagging of endogenous genes in a Toxoplasma gondii strain lacking Ku80. Eukaryotic Cell, 8(4), 530-539. doi:10.1128/EC.00358-08 Jacot, D., Daher, W., \& Soldati-Favre, D. (2013). Toxoplasma gondii myosin F, an essential motor for centrosomes positioning and apicoplast inheritance. The EMBO Journal, 32(12), 1702-1716. doi:10.1038/emboj.2013.113

Kim, J. H., Lee, S.-R., Li, L.-H., Park, H.-J., Park, J.-H., Lee, K. Y., Kim, M.-K., Shin, B. A., \& Choi, S.-Y. (2011). High cleavage efficiency of a 2A peptide derived from porcine teschovirus-1 in human cell lines, zebrafish and mice. PLOS ONE, 6(4), e18556. doi:10.1371/journal.pone.0018556

Kozak, M. (1987a). An analysis of 5'-noncoding sequences from 699 vertebrate messenger RNAs. Nucleic Acids Research, 15(20), 8125-8148. doi:10.1093/nar/15.20.8125 
Kozak, M. (1987b). At least six nucleotides preceding the AUG initiator codon enhance translation in mammalian cells. Journal of Molecular Biology, 196(4), 947-950. doi:10.1016/0022-2836(87)90418-9

Kremer, J. R., Mastronarde, D. N., \& McIntosh, J. R. (1996). Computer visualization of threedimensional image data using IMOD. Journal of Structural Biology, 116(1), 71-76. doi:10.1006/jsbi.1996.0013

Leung, J. M., Rould, M. A., Konradt, C., Hunter, C. A., \& Ward, G. E. (2014). Disruption of TgPHIL1 alters specific parameters of Toxoplasma gondii motility measured in a quantitative, three-dimensional live motility assay. PLOS ONE, 9(1), e85763. doi:10.1371/journal.pone.0085763

Lorestani, A., Ivey, F. D., Thirugnanam, S., Busby, M. A., Marth, G. T., Cheeseman, I. M., \& Gubbels, M. J. (2012). Targeted proteomic dissection of Toxoplasma cytoskeleton sub-compartments using MORN1. Cytoskeleton, 69(12), 1069-85. doi:10.1002/cm.21077

Lourido, S., Tang, K., \& Sibley, L. D. (2012). Distinct signalling pathways control Toxoplasma egress and host-cell invasion. The EMBO Journal, 31(24), 4524-4534. doi:10.1038/emboj.2012.299

Magno, R. C., Lemgruber, L., Vommaro, R. C., De Souza, W., \& Attias, M. (2005). Intravacuolar network may act as a mechanical support for Toxoplasma gondii inside the parasitophorous vacuole. Microscopy Research and Technique, 67(1), 45-52. doi:10.1002/jemt.20182

Makkonen, M., Bertling, E., Chebotareva, N. A., Baum, J., \& Lappalainen, P. (2013). Mammalian and malaria parasite cyclase-associated proteins catalyze nucleotide 
exchange on G-actin through a conserved mechanism. Journal of Biological Chemistry, 288(2), 984-994. doi:10.1074/jbc.M112.435719

Mattila, P. K., Quintero-Monzon, O., Kugler, J., Moseley, J. B., Almo, S. C., Lappalainen, P., \& Goode, B. L. (2004). A high-affinity interaction with ADP-actin monomers underlies the mechanism and in vivo function of Srv2/cyclase-associated protein. Molecular Biology of the Cell, 15(11), 5158-5171. doi:10.1091/mbc.e04-06-0444

McCoy, J. M., Whitehead, L., van Dooren, G. G., \& Tonkin, C. J. (2012). TgCDPK3 regulates calcium-dependent egress of Toxoplasma gondii from host cells. PLoS Pathogens, 8(12), e1003066. doi:10.1371/journal.ppat.1003066

Mehta, S., \& Sibley, L. D. (2011). Actin depolymerizing factor controls actin turnover and gliding motility in Toxoplasma gondii. Molecular Biology of the Cell, 22(8), 1290-

Muñiz-Hernández, S., González del Carmen, M., Mondragón, M., Mercier, C., Cesbron, M. F., Mondragón-González, S. L., González, S., \& Mondragón, R. (2011). Contribution of the residual body in the spatial organization of Toxoplasma gondii tachyzoites within the parasitophorous vacuole. BioMed Research International. doi:10.1155/2011/473983 nucleotide sequences around the translation initiation codon in eukaryote genomes. Nucleic Acids Research, 36(3), 861-871. doi:10.1093/nar/gkm1102

Ono, S. (2013). The role of cyclase-associated protein in regulating actin filament dynamics more than a monomer-sequestration factor. J Cell Sci, 126(15), 3249-3258. doi:10.1242/jcs.128231

Peng, D., \& Tarleton, R. (2015). EuPaGDT: a web tool tailored to design CRISPR guide RNAs for eukaryotic pathogens. Microbial Genomics, 1(4). doi:10.1099/mgen.0.000033 
1276

1277

1278

1279

1280

1281

1282

1283

1284

1285

1286

1287

1288

1289

1290

1291

1292

1293

1294

1295

1296

1297

1298

Periz, J., Whitelaw, J., Harding, C., Gras, S., Minina, M. I. D. R., Latorre-Barragan, F., Lemgruber, L., Reimer, M. A., Insall, R., Heaslip, A., \& Meissner, M. (2017). Toxoplasma gondii F-actin forms an extensive filamentous network required for material exchange and parasite maturation. ELife, 6, e24119. doi:10.7554/eLife.24119

Plattner, F., Yarovinsky, F., Romero, S., Didry, D., Carlier, M.-F., Sher, A., \& Soldati-Favre, D. (2008). Toxoplasma profilin is essential for host cell invasion and TLR11-dependent induction of an interleukin-12 response. Cell Host \& Microbe, 3(2), 77-87. doi:10.1016/j.chom.2008.01.001

Pospich, S., Kumpula, E.-P., Ecken, J. von der, Vahokoski, J., Kursula, I., \& Raunser, S. (2017). Near-atomic structure of jasplakinolide-stabilized malaria parasite F-actin reveals the structural basis of filament instability. Proceedings of the National Academy of Sciences, 114(40), 201707506. doi:10.1073/pnas.1707506114

Puhka, M., Joensuu, M., Vihinen, H., Belevich, I., \& Jokitalo, E. (2012). Progressive sheet-totubule transformation is a general mechanism for endoplasmic reticulum partitioning in dividing mammalian cells. Molecular Biology of the Cell, 23(13), 2424-2432. doi:10.1091/mbc.E10-12-0950

Reese, M. L., Zeiner, G. M., Saeij, J. P. J., Boothroyd, J. C., \& Boyle, J. P. (2011). Polymorphic family of injected pseudokinases is paramount in Toxoplasma virulence. Proceedings of the National Academy of Sciences of the United States of America, 108(23), 96259630. doi:10.1073/pnas.1015980108

Rommereim, L. M., Bellini, V., Fox, B. A., Pètre, G., Rak, C., Touquet, B., Aldebert, D., Dubremetz, J.-F., Cesbron-Delauw, M.-F., Mercier, C., \& Bzik, D. J. (2016). Phenotypes 
associated with knockouts of eight dense granule gene loci (GRA2-9) in virulent

Sahoo, N., Beatty, W., Heuser, J., Sept, D., \& Sibley, L. D. (2006). Unusual kinetic and structural properties control rapid assembly and turnover of actin in the parasite Toxoplasma gondii. Mol Biol Cell, 17(2), 895-906. doi:10.1091/mbc.E05-06-0512 Toxoplasma gondii coronin, an actin-binding protein that relocalizes to the posterior pole of invasive parasites and contributes to invasion and egress. The FASEB Journal, 28(11), 4729-4747. doi:10.1096/fj.14-252569

Sato, Y., Hliscs, M., Dunst, J., Goosmann, C., Brinkmann, V., Montagna, G. N., \& Matuschewski, K. (2016). Comparative Plasmodium gene overexpression reveals distinct perturbation of sporozoite transmission by profilin. Molecular Biology of the Cell, 27(14), 2234-2244. doi:10.1091/mbc.E15-10-0734

Schroeder, L. K., Barentine, A. E. S., Merta, H., Schweighofer, S., Zhang, Y., Baddeley, D., Bewersdorf, J., \& Bahmanyar, S. (2019). Dynamic nanoscale morphology of the ER surveyed by STED microscopy. The Journal of Cell Biology, 218(1), 83-96. doi:10.1083/jcb.201809107

Seeber, F. (1997). Consensus sequence of translational initiation sites from Toxoplasma gondii genes. Parasitology Research, 83(3), 309-311. doi:10.1007/s004360050254

1318 Shaw, M. K., \& Tilney, L. G. (1999). Induction of an acrosomal process in Toxoplasma gondii: Visualization of actin filaments in a protozoan parasite. Proceedings of the National Academy of Sciences of the United States of America, 96(16), 9095-9099. doi:10.1073/pnas.96.16.9095 
1322

1323

1324

1325

1326

1327

1328

1329

1330

1331

1332

1333

1334

1335

1336

1337

1338

1339

1340

1341

1342

1343

1344

1345

Sheffield, H. G., \& Melton, M. L. (1968). The fine structure and reproduction of Toxoplasma gondii. The Journal of Parasitology, 54(2), 209-226. doi:10.2307/3276925

Shen, B., Brown, K. M., Lee, T. D., \& Sibley, L. D. (2014). Efficient gene disruption in diverse strains of Toxoplasma gondii using CRISPR/CAS9. MBio, 5(3). doi:10.1128/mBio.01114-14

Silva, D. A. O., Lobato, J., Mineo, T. W. P., \& Mineo, J. R. (2007). Evaluation of serological tests for the diagnosis of Neospora caninum infection in dogs: Optimization of cut off titers and inhibition studies of cross-reactivity with Toxoplasma gondii. Veterinary Parasitology, 143(3), 234-244. doi:10.1016/j.vetpar.2006.08.028

Skillman, K. M., Diraviyam, K., Khan, A., Tang, K., Sept, D., \& Sibley, L. D. (2011). Evolutionarily divergent, unstable filamentous actin is essential for gliding motility in apicomplexan parasites. PLoS Pathogens, 7(10), e1002280. doi:10.1371/journal.ppat.1002280

Swapna, L. S., \& Parkinson, J. (2017). Genomics of apicomplexan parasites. Critical Reviews in Biochemistry and Molecular Biology, 52(3), 254-273. doi:10.1080/10409238.2017.1290043

Tomavo, S., Slomianny, C., Meissner, M., \& Carruthers, V. B. (2013). Protein trafficking through the endosomal system prepares intracellular parasites for a home invasion. PLoS Pathogens, 9(10). doi:10.1371/journal.ppat.1003629

Tosetti, N., Dos Santos Pacheco, N., Soldati-Favre, D., \& Jacot, D. (2019). Three F-actin assembly centers regulate organelle inheritance, cell-cell communication and motility in Toxoplasma gondii. ELife, 8. doi:10.7554/eLife.42669

Treeck, M., Sanders, J. L., Gaji, R. Y., LaFavers, K. A., Child, M. A., Arrizabalaga, G., Elias, J. E., \& Boothroyd, J. C. (2014). The calcium-dependent protein kinase 3 of Toxoplasma 
influences basal calcium levels and functions beyond egress as revealed by quantitative phosphoproteome analysis. PLoS Pathog, 10(6), e1004197. doi:10.1371/journal.ppat.1004197

Vaishnava, S., \& Striepen, B. (2006). The cell biology of secondary endosymbiosis - how parasites build, divide and segregate the apicoplast. Molecular Microbiology, 61(6), 1380-1387. doi:10.1111/j.1365-2958.2006.05343.x

West, M., Zurek, N., Hoenger, A., \& Voeltz, G. K. (2011). A 3D analysis of yeast ER structure reveals how ER domains are organized by membrane curvature. The Journal of Cell Biology, 193(2), 333-346. doi:10.1083/jcb.201011039 Andenmatten, N., Nelson, S. R., Warshaw, D. M., Ward, G. E., \& Meissner, M. (2017). Surface attachment, promoted by the actomyosin system of Toxoplasma gondii is important for efficient gliding motility and invasion. BMC Biology, 15(1). doi:10.1186/s12915-016-0343-5 


\section{Figure legends}

\section{Figure 1: Alternative translational start sites lead to the generation of two different CAP} isoforms.

(A) Sequence alignment of the first 57 amino acid residues of TgCAP with that of other Apicomplexa. Green shading indicates cysteines which are putative palmitoylation sites, yellow shading indicates methionines which are putative alternative translational start sites. (B) Subcellular localisation of a CAP-GFP fusion. Scale bar, $2 \mu \mathrm{m}$. (C) Subcellular localisation of CAP by immunofluorescence assay (IFA) using rabbit anti-TgCAP antibodies. Scale bar, $2 \mu \mathrm{m}$. (D) Western blot of CAP expression levels over the first 24 hours following host cell invasion using anti-TgCAP antibodies. Anti-Toxoplasma antibodies were used as a loading control. (E) Alignment of the Toxoplasma consensus translation initiation (Kozak) sequence (Seeber, 1997) with the translation initiation sequences of CAP's first (M1) and second (M37) putative translational start sites. Green shading indicates bases that correspond to the Kozak sequence. (F) Schematic of TgCAP with annotations for the two putative CAP isoforms and the mutation experiments performed to test their expression: Mutation of $M 1$ to leucine $(L)$ to produce shortCAP, and mutation of M37 to $L$ producing longCAP. Green diamonds indicate putative palmitoylation sites. (G) IFA and (H) western blot of ectopic HA-tagged TgCAP isoforms and cysteine mutants (C6 and C8). Inclusion of a HA-tag makes the protein run more slowly than the untagged protein. Anti-Toxoplasma antibodies were used as a loading control in $(H)$. Scale bar in $(G), 5 \mu \mathrm{m}$.

\section{Figure 2: A second generation RH $\Delta k u 80$ DiCre_T2A parasite strain stably expresses DiCre.}

(A) Schematic of the DiCre_T2A expression construct. The chloramphenicol resistance cassette (CAT) is flanked by T2A skip peptides. The two Cre subunits (FRB_Cre2 and FKBP_Cre1) are located on either side of the T2A::CAT::T2A cassette. The fusion protein is driven by the alpha-tubulin promotor with a SAG1 3' UTR. The red hexagon indicates the position of the stop-codon. (B) Flow cytometry analysis to determine excision efficiency of the $\mathrm{RH}$ DiCre_T2A $\Delta k u 80$ line following 65 days of frequent extracellular stress. Excision is determine $\bar{d}$ by a shift from Killer Red ${ }^{(+)}$to Killer $\operatorname{Red}^{(+)}$and $\mathrm{YFP}^{(+)}$expression. Parasites were analysed $22 \mathrm{~h}$ after induction with $50 \mathrm{nM}$ rapamycin (RAP) for $4 \mathrm{~h}$. Due to analysing $22 \mathrm{~h}$ after induction of excision, parasites still have residual KillerRed signal. (C) Table summarizing the excision efficiency of RAP treated RH DiCre_T2A $\Delta k u 80$ parasites over time in the presence or absence of chloramphenicol selection. "Day" refers to the number of days in cell culture while "rep" corresponds to biological replicates.

\section{Figure 3: Generation of CAP conditional knockout and complementation strains}

(A) Schematic of the CAP conditional knock out strategy using double homologous integration. The position of the 5' LoxP site in the CAP promotor is indicated as well as the predicted sizes of the PCR amplicons. (B) Agarose gel showing the expected PCR products for correct integration at the endogenous locus. (C) IFA $46 \mathrm{hr}$ after treatment with DMSO or $50 \mathrm{nM}$ rapamycin for $4 \mathrm{~h}$. Scale bar, $5 \mu \mathrm{m}$. (D) Western blot showing absence of CAP protein in the cloned $\triangle$ CAP parasites using anti-TgCAP antibodies. Anti-Toxoplasma antibodies were used as a loading control. (E) Subcellular localisation and (F) Western blot of ectopic HA-tagged $T g$ CAP isoforms, in the $\triangle$ CAP background. Inclusion of a HA-tag makes the protein run more slowly than the untagged protein. IFA images have been individually contrast adjusted to aid in visualising protein localisation. Scale bar in (E), $5 \mu \mathrm{m}$. Anti-Toxoplasma antibodies were used as a loading control in (F).

Figure 4: CAP plays an important but not essential role during the lytic cycle in cell culture 
(A) Overview of the flow cytometry competition assay (left). Competition assays of mCherryexpressing $\triangle \mathrm{CAP}$ and CAP complementation lines with non-fluorescent WT-complemented parasites (right). The ratio of $\mathrm{mCherry}^{(+)}$to $\mathrm{mCherry}^{(-)}$parasites was analysed by flow cytometry at day 0,15 and 30. Data are represented as mean \pm s.d. (D0 and D30, $n=6$. D15, $n=4$ ). Twoway ANOVA followed by a multiple comparison Sidak's test was used to compare means between time points. (B) 3D Matrigel-based motility assays performed in the absence of inducers of motility. Results are expressed as mean \pm s.d. $(n=4)$. Each data point corresponds to a single technical replicate from one of four independent biological replicates, on which significance was assessed using an unpaired $t$-test. (C) Invasion assay comparing $\triangle C A P$ to the complemented lines. Data are represented as mean \pm s.d. $(n=3)$. One-way ANOVA followed by Dunnett's test was used to compare means to the $\triangle C{ }^{C P^{C A P}}$ mean (D) Egress assay. Graph shows number of egressed vacuoles in response to BIPPO over time. Data are represented as mean \pm s.d. $(n=3)$. Two-way ANOVA followed by Dunnett's test was used to compare means to the $\triangle C A P^{C A P}$ mean. Stated significance is in comparison to $\triangle C A P^{C A P}$. (E) Dense granule trafficking assay. $\triangle \mathrm{CAP}$ and CAP complemented lines were transiently transfected with SAG1 $\triangle \mathrm{GPI}$-mCherry that allows visualisation of dense granules. The length and speed of directed runs were recorded using fluorescence microscopy and analysed using ImageJ. Data are represented as mean \pm s.d. $(n=3)$. One-way ANOVA followed by Dunnett's test was used to compare means to the $\triangle C A P^{C A P}$ mean.

\section{Figure 5: CAP is important for rosetting and rapid cell-cell communication}

(A) Representative fluorescence images of mCherry expressing $\triangle \mathrm{CAP}$ parasites and complemented isoforms (left). Quantification of rosetting vs. non-rosetting parasites (right). Data are represented as mean \pm s.d. $(n=2)$. One-way ANOVA followed by Dunnett's test was used to compare means to the $\triangle C A P^{C A P}$ mean. (B-E) FRAP experiments to measure transfer of mCherry between individual parasites in a vacuole. (B) Heatmaps showing the percentage change in fluorescence of individual parasites in (i) CAP complemented, (ii) $\triangle \mathrm{CAP}$ and (iii) $\Delta$ gra2 parasite lines. A recovery plot and image for representative vacuoles are included. Regions of interest are numbered; the bleached parasite is "1". Numbers are allocated based on proximity to the bleached parasite. The yellow lightning bolt indicates which parasite was photobleached. Data are representative of two independent experiments. (C, D) Graphs quantifying the type $(C)$ and amount $(D)$ of recovery for all parasite lines. Data are represented as mean \pm s.d. ( $\triangle \mathrm{CAP}$ and $\triangle \mathrm{CAP}^{\mathrm{CAP}} n=4$, all other lines, $n=2$ ). Statistical significance was assessed by either Chi-square test (D) or one-way ANOVA followed by Dunnett's test to compare means to the $\triangle$ CAP ${ }^{C A P}$ mean (C). (E) FRAP analysis of $\triangle$ CAP parasites. The images and graphs represent sequential photobleaching and recovery measurements of individual parasites within the vacuole. The yellow lightning bolt indicates which parasite was photobleached. The white arrow identifies the parasite from which the majority of recovery was observed. All scale bars, $5 \mu \mathrm{m}$.

\section{Figure 6: CAP is important for daughter cell orientation, but not synchronous division} (A) Parasites stained with anti-IMC 3 antibodies to visualise daughter cell orientation and division. Scale bar, $5 \mu \mathrm{m}$. (B) Quantification of synchronicity of division in parasite vacuoles using daughter cell staining from (A) reveals no defect in the synchronicity of $\triangle$ CAP parasites. Data are represented as mean \pm s.d. $(n=3)$. Significance was assessed using an unpaired twotailed $t$-test. (C) Quantification of daughter cell orientation in parasite vacuoles reveals a significant defect in the daughter cell orientation of $\triangle \mathrm{CAP}$ parasites. Data are represented as mean \pm s.d. $(n=3)$. One-way ANOVA followed by Dunnett's test was used to compare means to the $\triangle \mathrm{CAP}^{\mathrm{CAP}}$ mean.

\section{Figure 7: CAP KO parasites are still connected by a decentralised residual body}

(A) FRAP analysis of $\triangle C A P^{C A P}$ parasites. The images and graphs represent sequential photobleaching and recovery measurements of individual parasites within the vacuole. (B) (i) 
FIB SEM of the vacuole from (A) with a 3D model highlighting the residual body (green skeleton representing how the approximate centre can be traced through to the basal poles) and parasite openings at the basal pole (orange ring). Note that the illustrated connections display the distance between the parasite's posterior ends, and do not represent tubular connections between them. Numbering of parasites is consistent with (A). (ii) Zoomed image of the residual body from (i), (iii) zoomed image of the residual body from (i) overlaid with the 3D model. (C) FRAP analysis of $\triangle$ CAP parasites. The images and graphs represent sequential photobleaching and recovery measurements of individual parasites within the vacuole. (D) (i) FIB SEM of the vacuole from (A), numbering of parasites is consistent with (C). (ii) Zoomed image of the boxed region from (i). Green arrows indicate the residual body (left panel). A 3D model highlights the residual body (green skeleton drawn through the lumen of the connections between parasites), parasite openings at the basal pole (orange ring) (right panel). (iii) A 3D model of selected features in the vacuole from (C), highlighting the residual body (green), parasite openings at the basal pole (orange ring) and coarse segmentation of two of the parasites (yellow) (left panel). This model is shown with an orthoslice from the FIB SEM volume (right panel). (E) Zoomed images of the boxed region from (Di). Blue arrows indicate a putative ER structure (left). A 3D model highlights this putative ER (blue) and parasite openings at the basal pole (orange ring) (right). (i) View facing a $Z$ orthoslice. (ii) View facing an $X$ orthoslice. The yellow lightning bolt indicates which parasite was photobleached and the white arrow identifies the parasite from which recovery was observed $(A, C)$. Scale bar, $5 \mu \mathrm{m}(A, C)$ or 1 $\mu \mathrm{m}(\mathrm{B}, \mathrm{D}, \mathrm{E})$.

Figure 8: CAP is essential for virulence in type II but not type I parasites. Survival rates of C57BL/6 mice infected with 50,000 Pru $\triangle \mathrm{CAP}$ parasites or with complementing CAP variants. (C) ELISA testing sera reactivity of naïve or Toxoplasma infected mice using Toxoplasma antigen. D) Cyst burden in the brain of C57BL/6 mice 32 days post-infection with 5,000 Pru $\triangle$ CAP parasites or with complementing CAP variants. For all experiments, five animals were infected for each strain.

\section{Supplementary Figure 1. Endogenous tagging of CAP}

(A) Schematic of CAP endogenous tagging strategy. Arrowhead indicates CRISPR/CAS9 cut site. (B) IFA of the resulting HA-tagged line. Scale bar, $2 \mu \mathrm{m}$.

\section{Supplementary Figure 2. Excision efficiency testing of RH DiCre $\Delta k u 80 \Delta h \times g p r t$}

Flow cytometry analysis to determine excision efficiency of the original RH DiCre $\Delta k u 80 \Delta h \times g p r t$ line following 65 days of standard, non-stressing, culture conditions. Excision is determined by a shift from Killer $\operatorname{Red}^{(+)}$to Killer $\operatorname{Red}^{(+)}$and $\mathrm{YFP}^{(+)}$expression. Parasites were analysed $22 \mathrm{~h}$ after induction with $50 \mathrm{nM}$ rapamycin (RAP) for $4 \mathrm{~h}$. Due to analysing $22 \mathrm{~h}$ after induction of excision, parasites still have residual KillerRed signal.

\section{Supplementary Figure 3. Excision testing of the CAP conditional knockout lines}

(A) Schematic of the CAP locus in the LoxPCAP conditional knockout lines. Rapamycin induced excision of CAP is detected by PCR3. (B) Diagnostic PCR of the CAP locus in the (i) LoxPCAP lines $5 \mathrm{~d}$ after treatment with either DMSO (D) or rapamycin (R). (ii) Excised clones were subsequently obtained from these rapamycin treated populations.

\section{Supplementary Figure 4. CAP-HA expression in the $\triangle$ CAP $^{\mathrm{CAP}}$ line}

Western blot on CAP expression levels in the $\triangle$ CAP ${ }^{\text {CAP }}$ strain over the first 24 hours following host cell invasion using anti-TgCAP antibodies. Anti-Toxoplasma antibodies were used as a loading control.

\section{Supplementary Figure 5. Replication analysis of $\triangle \mathrm{CAP}$ and the complemented lines.}


Data are represented as mean \pm s.d. $(n=2)$. One-way ANOVA followed by Dunnett's test was used to compare means to the $\triangle C A P^{C A P}$ mean. "Other" refers to vacuoles with a non-power of 2 number of parasites.

\section{Supplementary Figure 6. 3D Matrigel-based motility assays performed in the absence of inducers of motility.}

Results are expressed as mean \pm s.d. $(n=4)$. Each data point corresponds to a single technical replicate from one of four independent biological replicates, on which significance was assessed using an unpaired $t$-test.

\section{Supplementary Figure 7. Scanning electron micrographs of intracellular $\triangle$ CAP and complemented lines \\ Vacuole organisation is disrupted in $\triangle \mathrm{CAP}$ and $\triangle \mathrm{CAP} \mathrm{P}^{\text {longCAP }}$ parasite strains.}

\section{Supplementary Figure 8. Apicoplast segregation analysis}

Apicoplast segregation was not affected in the $\triangle$ CAP line. Representative IFA images (left) and quantification of apicoplast segregation (right). Data are represented as mean \pm s.d. $(n=3)$. Significance was assessed using an unpaired two-tailed $t$-test. Scale bar, $5 \mu \mathrm{m}$.

\section{Supplementary Figure 9. Close up FIB SEM images of parasite connections in the $\triangle$ CAP vacuole from (Fig. 7C-E)}

The 3D model highlights the residual body (green skeleton representing how the approximate centre can be traced through to the basal poles), parasite openings at the basal pole (orange ring) and coarse segmentation of two of the parasites (yellow). Scale bars are not shown since these are oblique views - see Fig. 7 and Methods for scale information.

\section{Video 1. Live egress imaging of a $\triangle C A P^{\mathrm{CAP}}$ vacuole}

Egress was induced by addition of $50 \mu \mathrm{M}$ BIPPO at $0 \mathrm{~s}$. Image taken every $1.8 \mathrm{~s}$. The video is played at $6.6 \mathrm{fps}$ and the time is indicated in seconds. Scale bar, $10 \mu \mathrm{m}$.

\section{Video 2. Live egress imaging of a $\triangle \mathrm{CAP}$ vacuole}

Egress was induced by addition of $50 \mu \mathrm{M}$ BIPPO at $0 \mathrm{~s}$. Image taken every $1.8 \mathrm{~s}$. The video is played at $6.6 \mathrm{fps}$ and the time is indicated in seconds. Scale bar, $10 \mu \mathrm{m}$.

\section{Video 3. Live egress imaging of a $\triangle C A P^{\text {shortCAP }}$ vacuole}

Egress was induced by addition of $50 \mu \mathrm{M}$ BIPPO at $0 \mathrm{~s}$. Image taken every $1.8 \mathrm{~s}$. The video is played at $6.6 \mathrm{fps}$ and the time is indicated in seconds. Scale bar, $10 \mu \mathrm{m}$.

\section{Video 4. Live egress imaging of a $\triangle C A P^{\text {longCAP }}$ vacuole}

Egress was induced by addition of $50 \mu \mathrm{M}$ BIPPO at $0 \mathrm{~s}$. Image taken every $1.8 \mathrm{~s}$. The video is played at $6.6 \mathrm{fps}$ and the time is indicated in seconds. Scale bar, $10 \mu \mathrm{m}$.

\section{Video 5. FRAP on a $\triangle$ CAP $^{\text {CAP }}$ vacuole}

A single parasite was photobleached at $3 \mathrm{~s}$. Images taken every $1 \mathrm{~s}$. The video is played at 2 fps and the time is indicated in seconds. Scale bar, $5 \mu \mathrm{m}$.

\section{Video 6. FRAP on a $\triangle$ CAP vacuole}

A single parasite was photobleached at $3 \mathrm{~s}$. Images taken every $1 \mathrm{~s}$. The video is played at 2 fps and the time is indicated in seconds. Scale bar, $5 \mu \mathrm{m}$.

\section{Video 7. FRAP on a $\triangle$ Gra2 vacuole}

A single parasite was photobleached at $3 \mathrm{~s}$. Images taken every $1 \mathrm{~s}$. The video is played at 2 fps and the time is indicated in seconds. Scale bar, $5 \mu \mathrm{m}$. 
1597 Video 8. FIB SEM of the $\triangle$ CAP ${ }^{\text {CAP }}$ vacuole from Fig. 7A-B with 3D model overlay

1598 All FIB SEM slices of the $\triangle \mathrm{CAP}^{\mathrm{CAP}}$ vacuole Fig. 7A-B. The 3D model highlights the residual 1599 body (green skeleton representing how the approximate centre can be traced through to the basal poles, along with a structure that extends from that centre but has no connection) and parasite openings at the basal pole (orange ring). The volume of the FIB SEM dataset shown is indicated in the Materials and methods.

\section{Video 9. FIB SEM of the $\triangle$ CAP vacuole from Fig. 7C-E with 3D model overlays} (green skeleton drawn through the approximate central axis of the lumen of the connections between parasites), parasite openings at the basal pole (orange ring), coarse segmentation of the central part of two of the parasites (yellow), and part of the putative ER (blue, note that only a selected region was segmented; from the inner face of two of the basal pores through part of the decentralised residual body). The volume of the FIB SEM dataset shown is indicated in the Materials and methods.

\section{Supplementary File 1. Primers listed in the Materials and methods}

\section{Supplementary File 2. Synthetic DNA listed in the Materials and methods}

\section{Supplementary File 3. BioWave program details for FIB SEM}




\section{Figure 1}

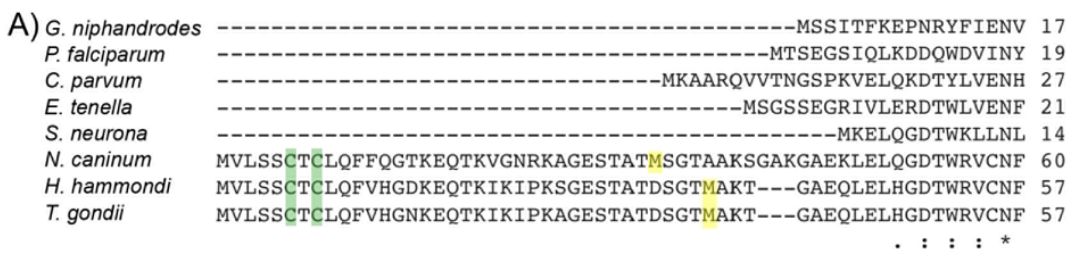

B)

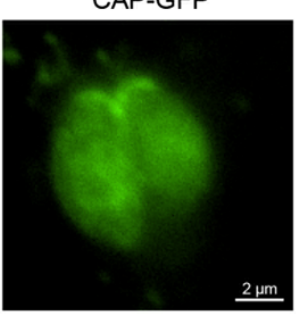

C)

$\mathrm{RH} \Delta k u 80 \mathrm{DiCre}$

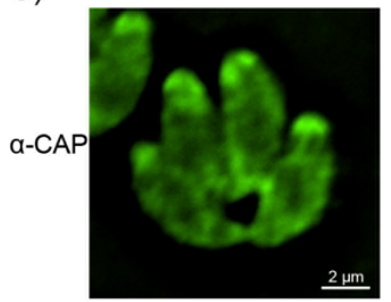

D)

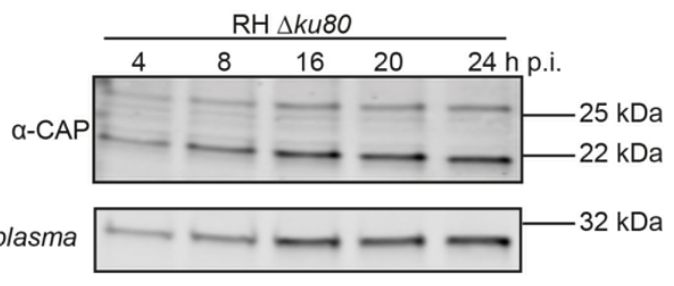

E) Position (relative to the ATG start codon)

\begin{tabular}{|c|c|c|c|c|c|c|c|c|c|c|}
\hline-6 & -5 & -4 & -3 & -2 & -1 & +1 & +2 & +3 & +4 & \\
\hline $\mathrm{g}$ & $\mathrm{N}$ & C & A & A & a & A & $\mathbf{T}$ & G & g & T. gondii translation initiation consensus sequence \\
\hline $\mathrm{g}$ & C & $a$ & $\mathrm{~g}$ & $\mathrm{~g}$ & a & A & $\mathbf{T}$ & G & $\mathrm{g}$ & TgCAP sequence around "M1" \\
\hline $\mathrm{g}$ & $\mathrm{g}$ & a & a & c & $a$ & A & $\mathbf{T}$ & G & g & TgCAP sequence around "M37" \\
\hline
\end{tabular}

F)

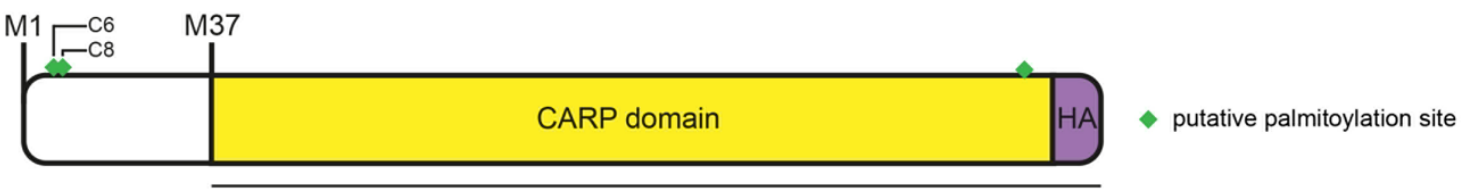

shortCAP (M1L, predicted $20 \mathrm{kDa})$

longCAP (M37L, predicted $23.9 \mathrm{kDa}$ )

G)

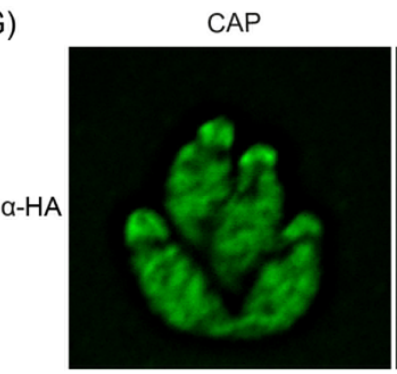

shortCAP

longCAP

CAP_C6,8A
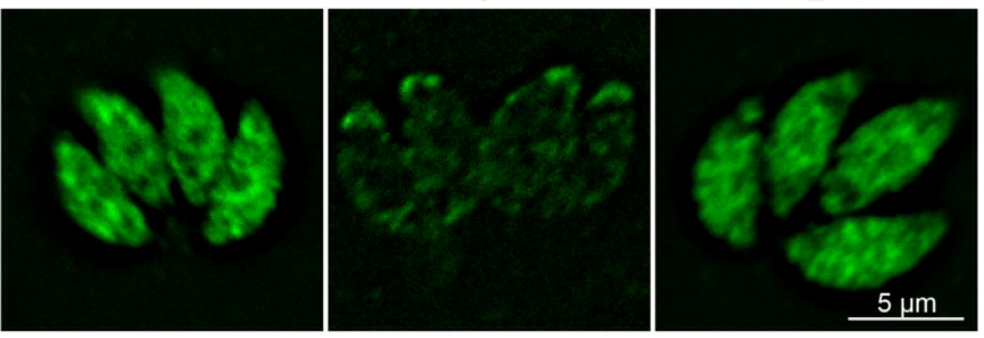

$\mathrm{H})$
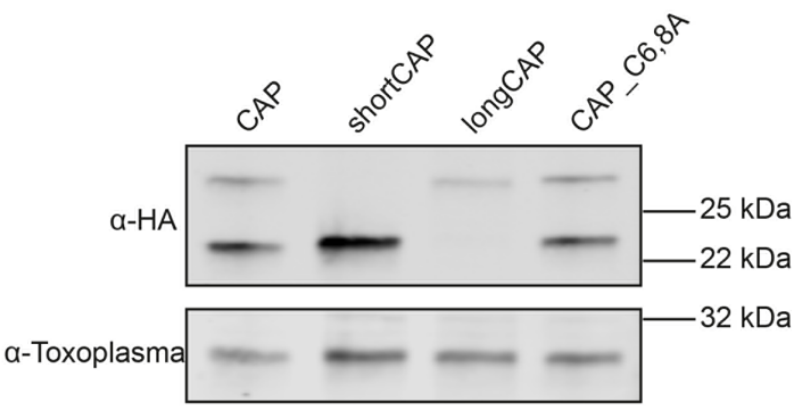
Figure 2

1626

A)

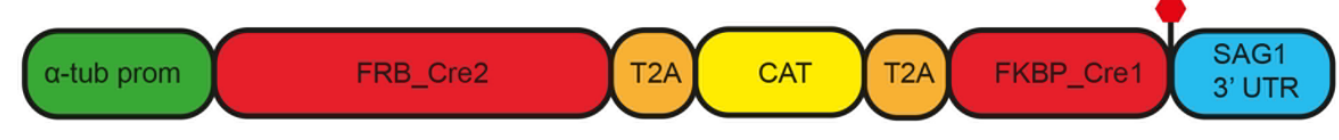

B)
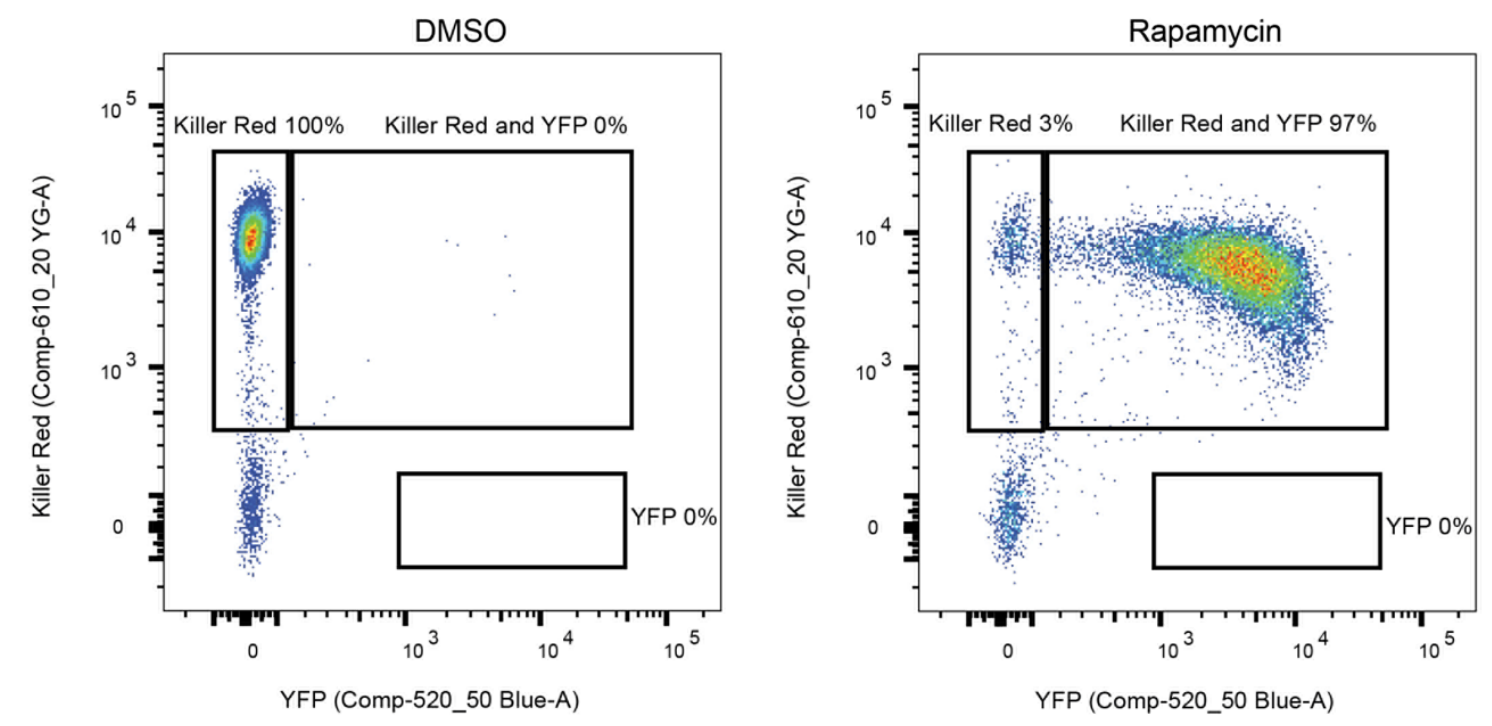

C)

No selection Chloramphenicol selection

1627

1628

1629

1630

1631

1632

1633

1634

1635

1636

1637

1638

1639

1640

1641

1642

1643

1644

1645 
Figure 3

1651

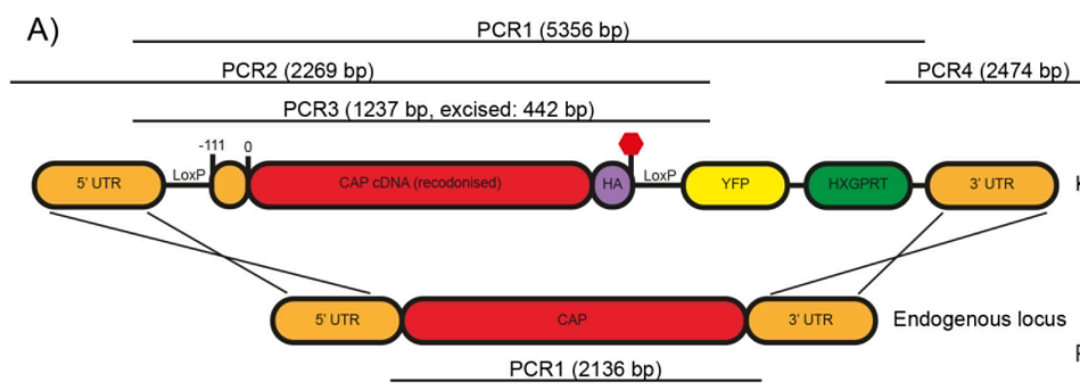

B)

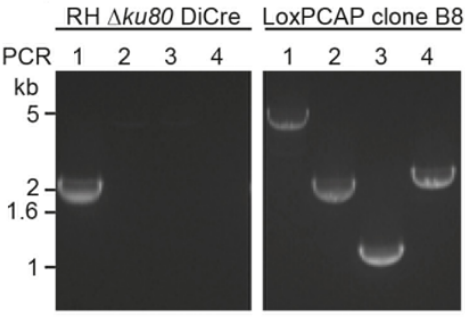

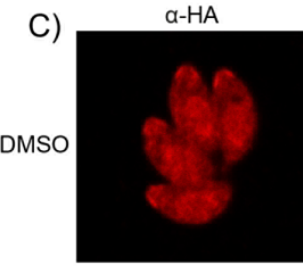
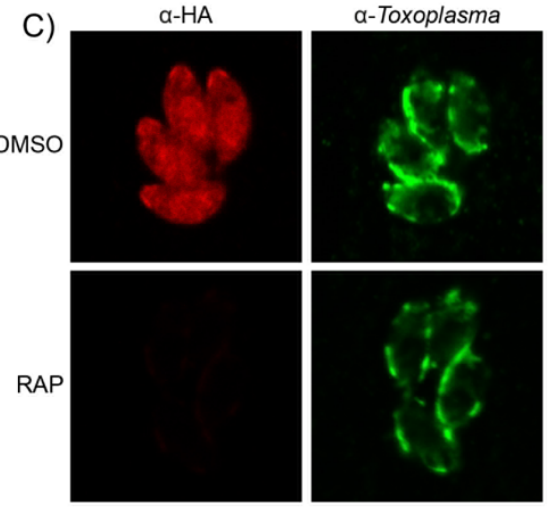

E)

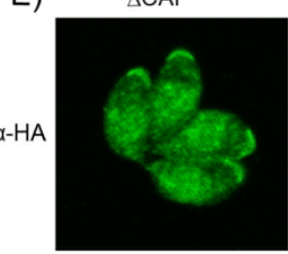

$\triangle C A P$ shortCAP

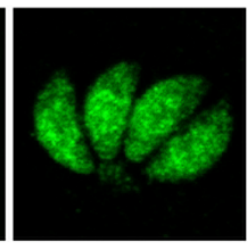

Merge
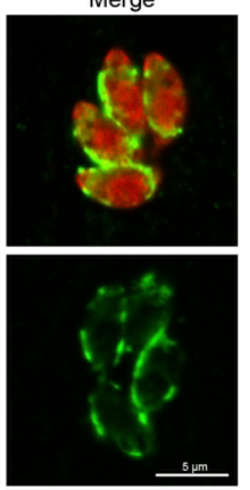

$\triangle C A P^{\text {longCAP }}$

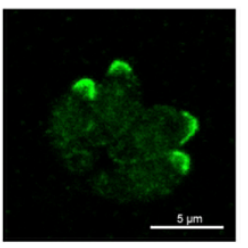

D)
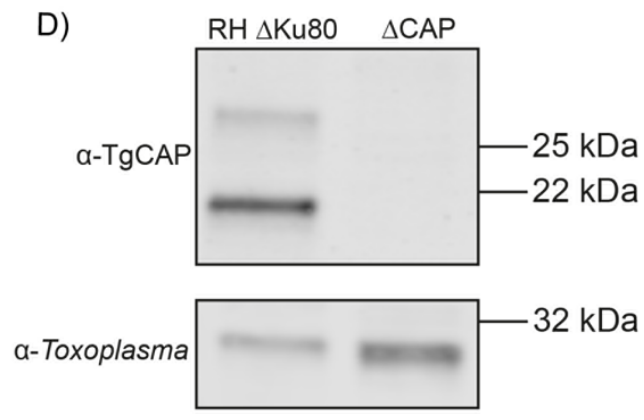

F)

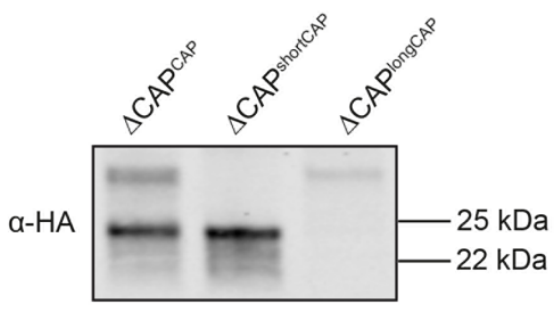

a-Toxoplasma

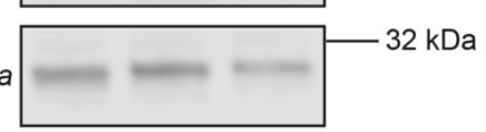


1671 Figure 4

A)
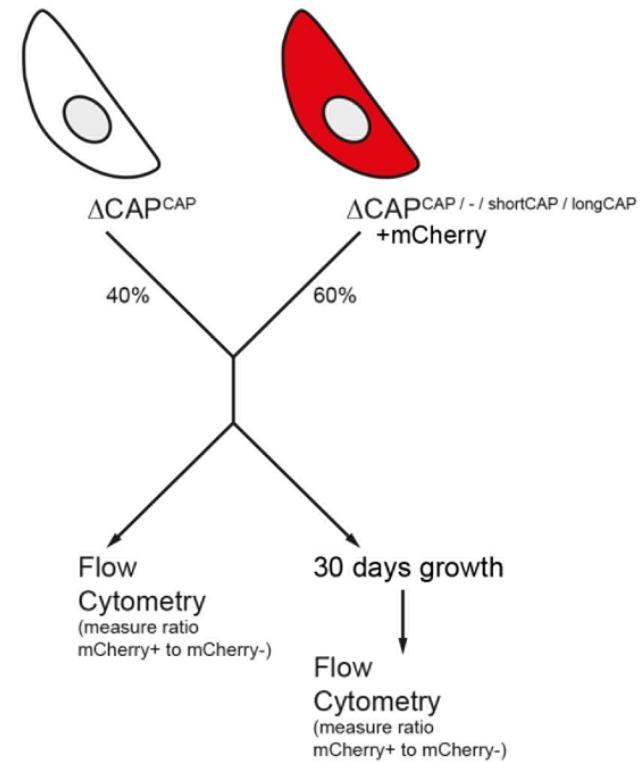

B)

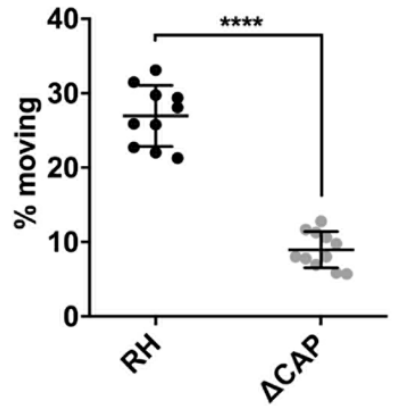

C)

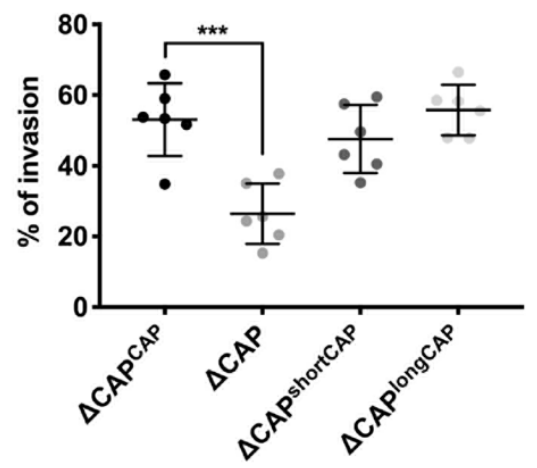

E)

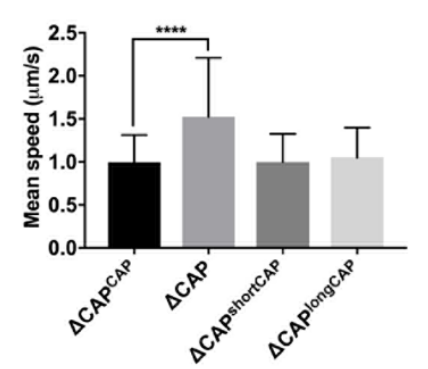

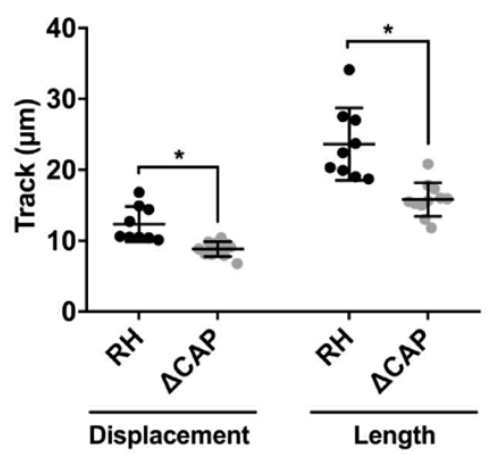

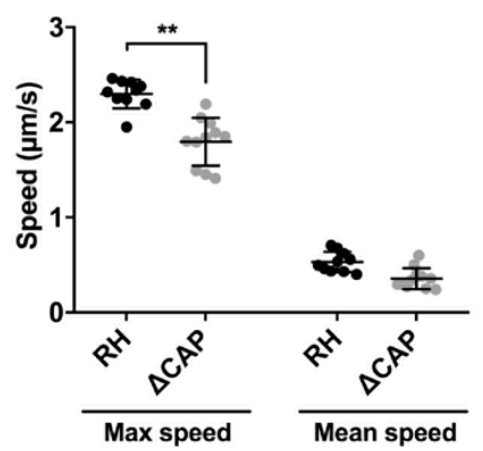

ํํㅀ

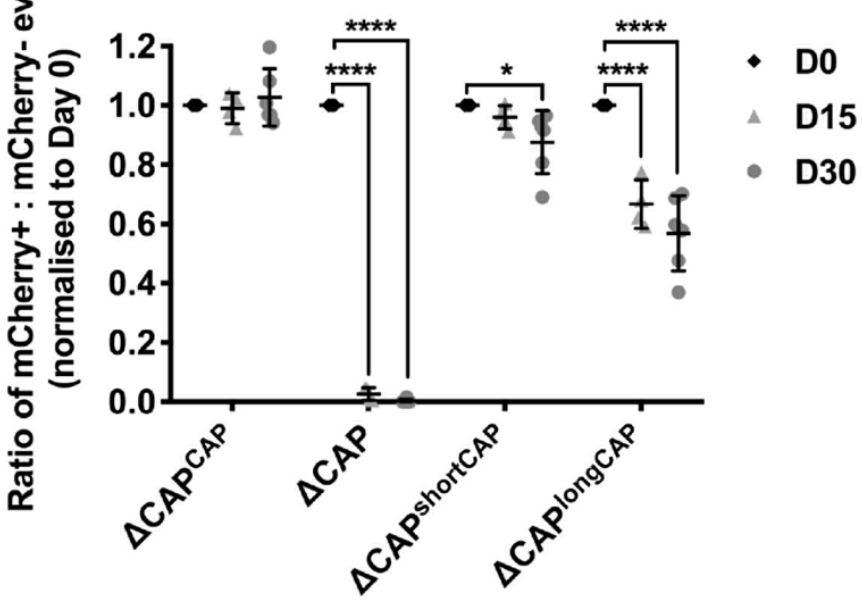

D)

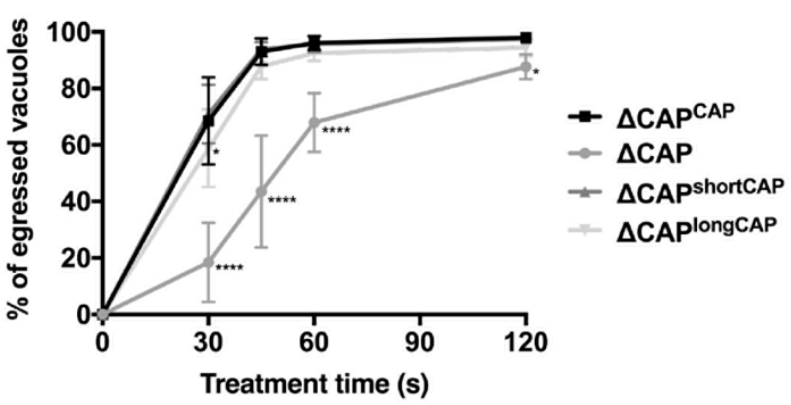




\section{$1674 \quad$ Figure 5}

A)
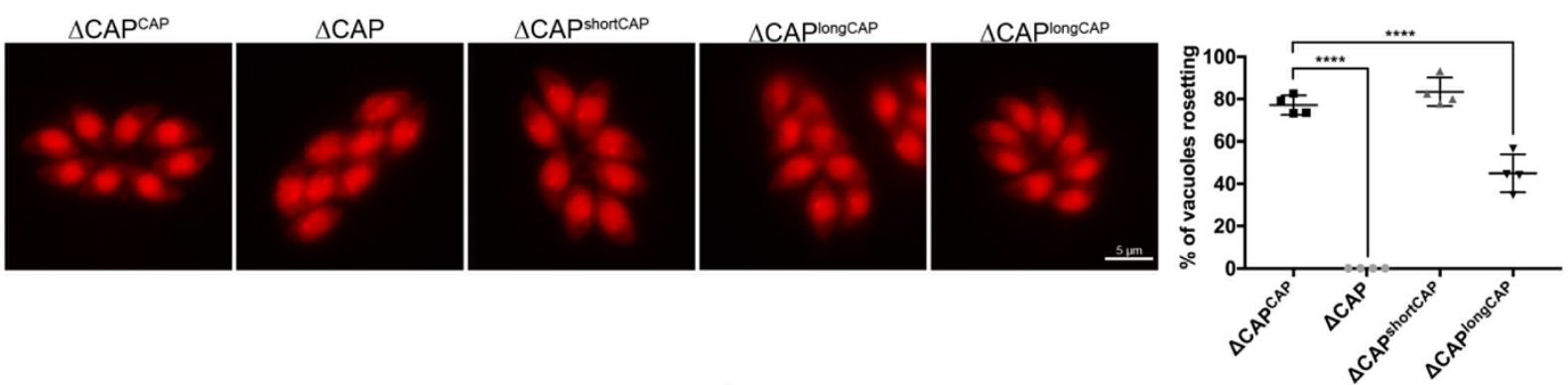

B) i)
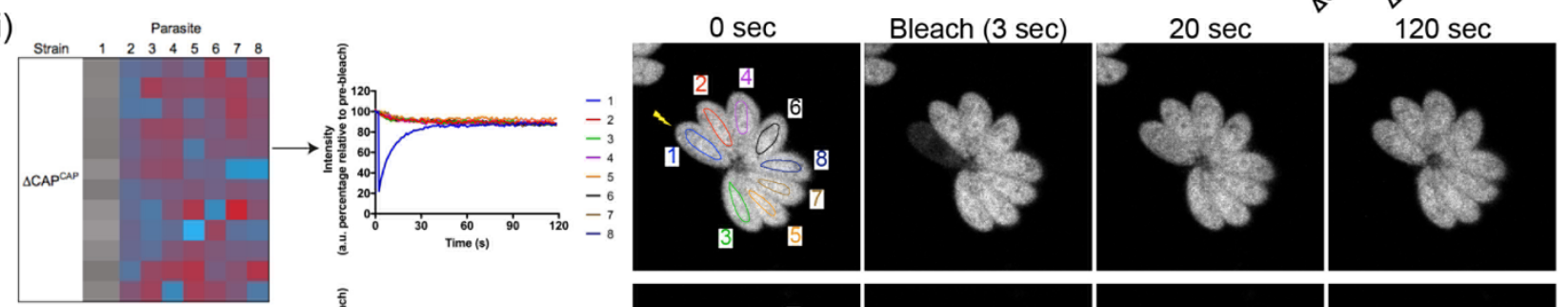

ii)

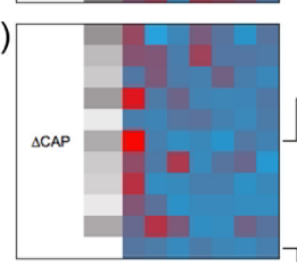

iii)
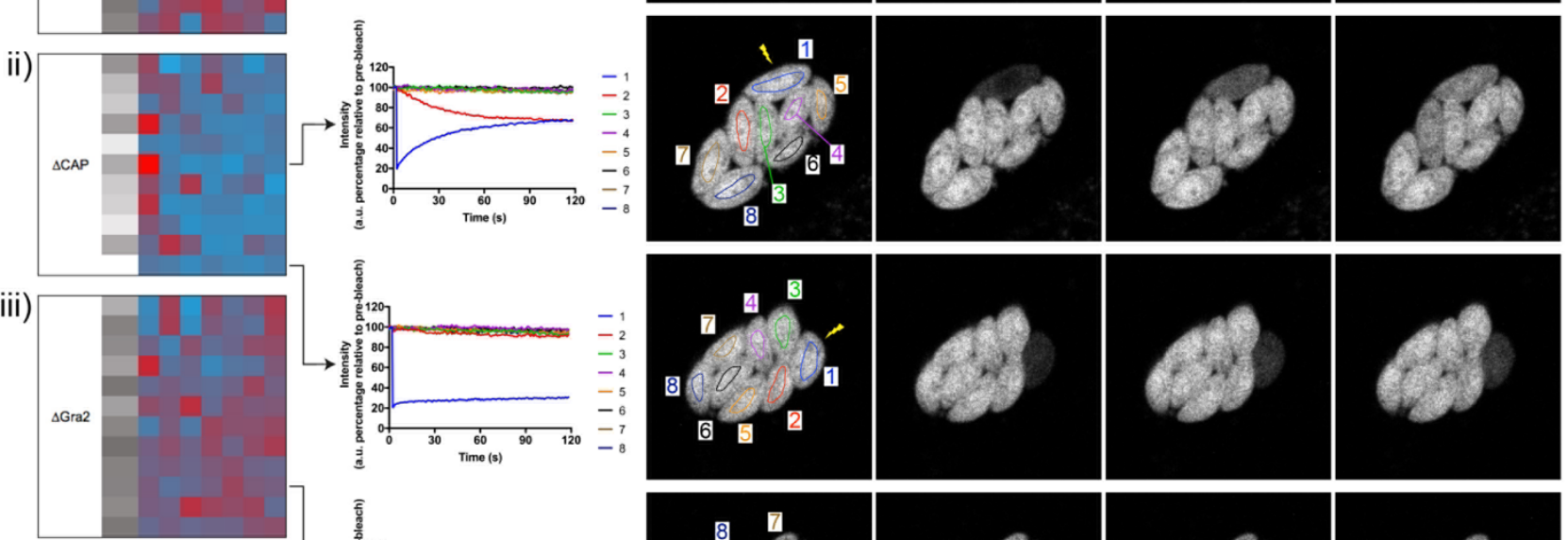

$\%$ recovery of bleached parasite $91 \% \quad 13 \%$ $\%$ loss of mCherry (material transferred)
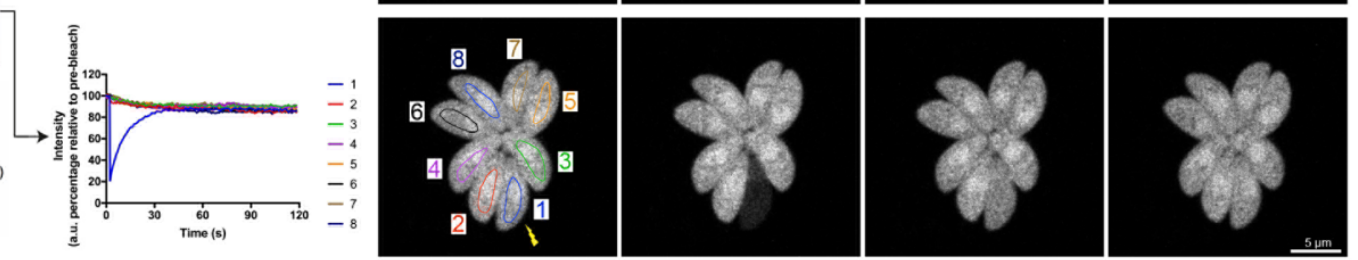

C)

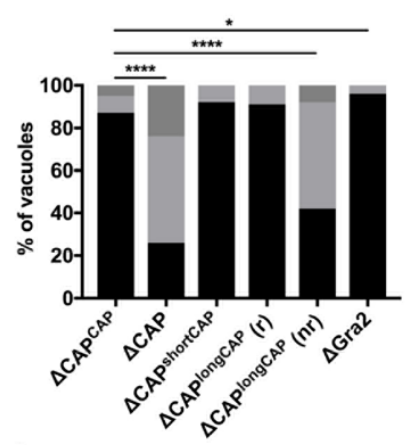

E)
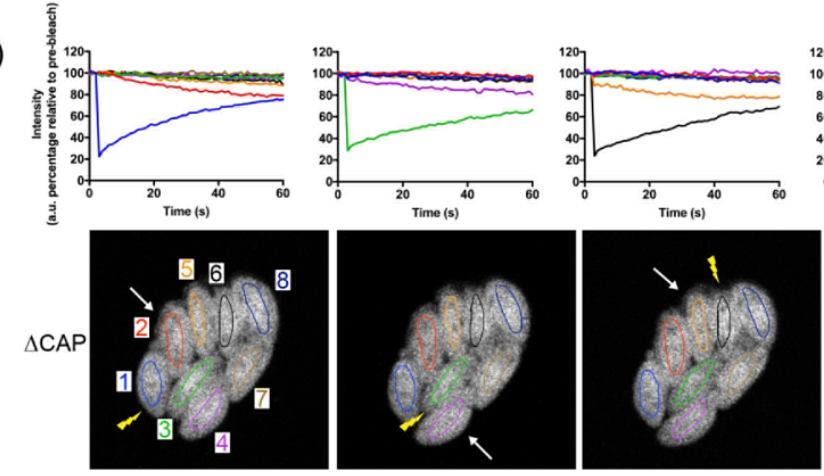

D)
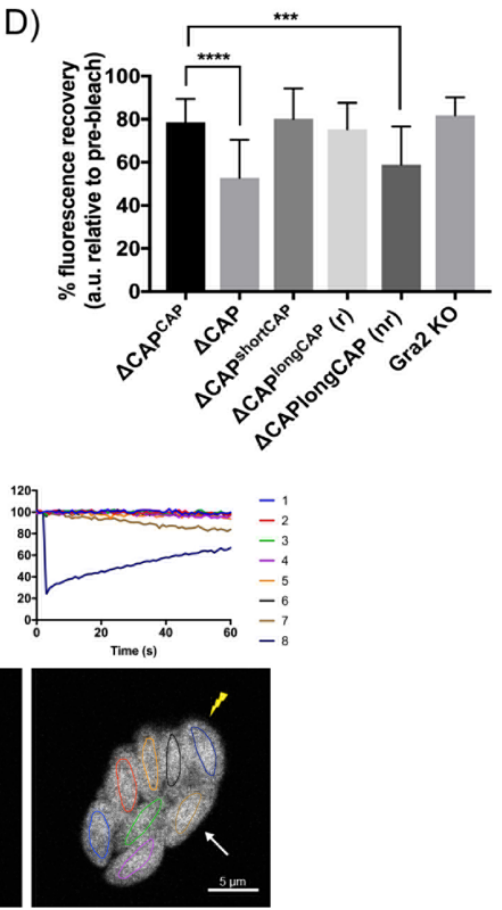
1675 Figure 6

A)
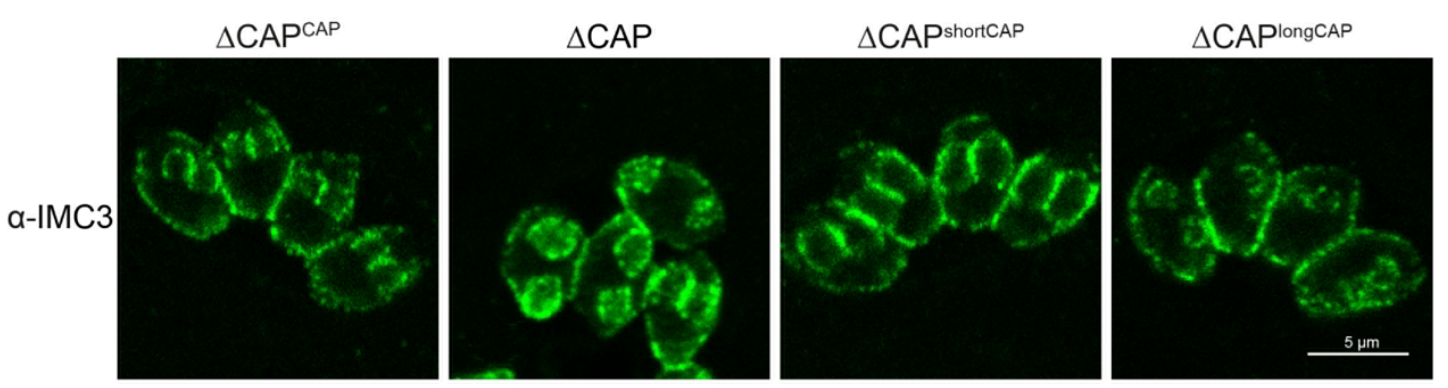

B)

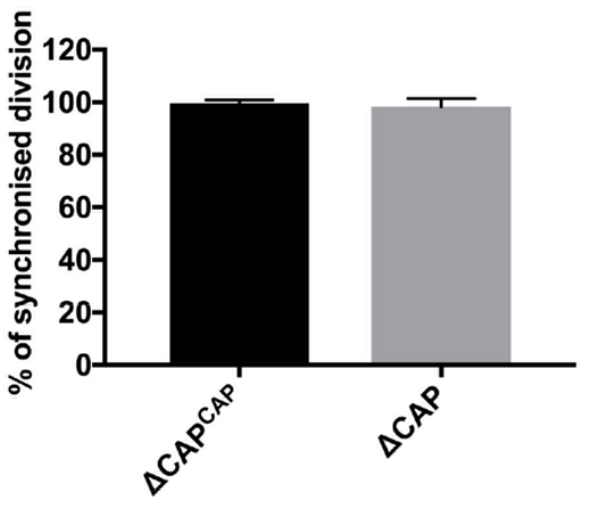

C)

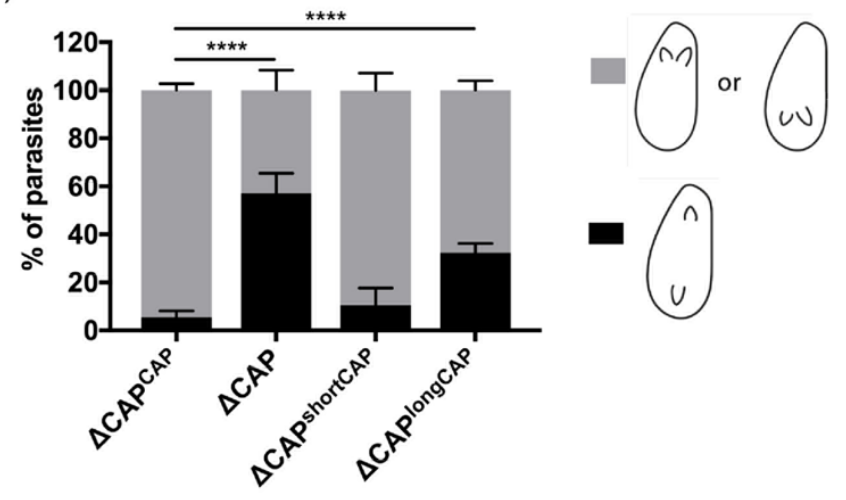

1676

1677

1678

1679

1680

1681

1682

1683

1684

1685

1686

1687

1688

1689

1690

1691

1692

1693

1694

1695

1696

1697

1698

1699

1700

1701

1702

1703

1704

1705

1706 


\section{$1707 \quad$ Figure 7}
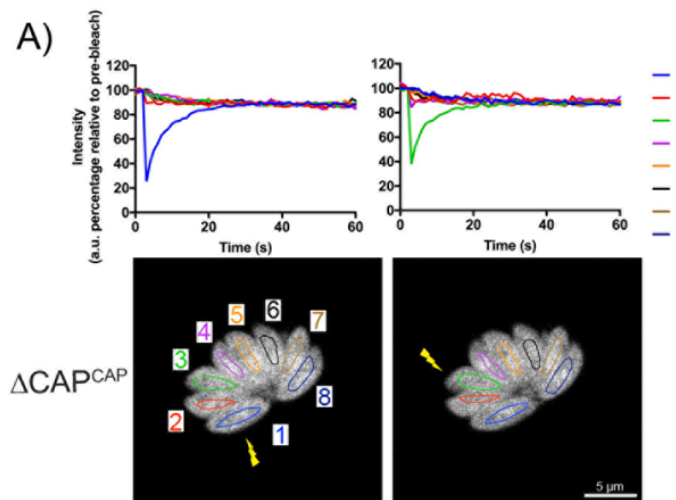

C)
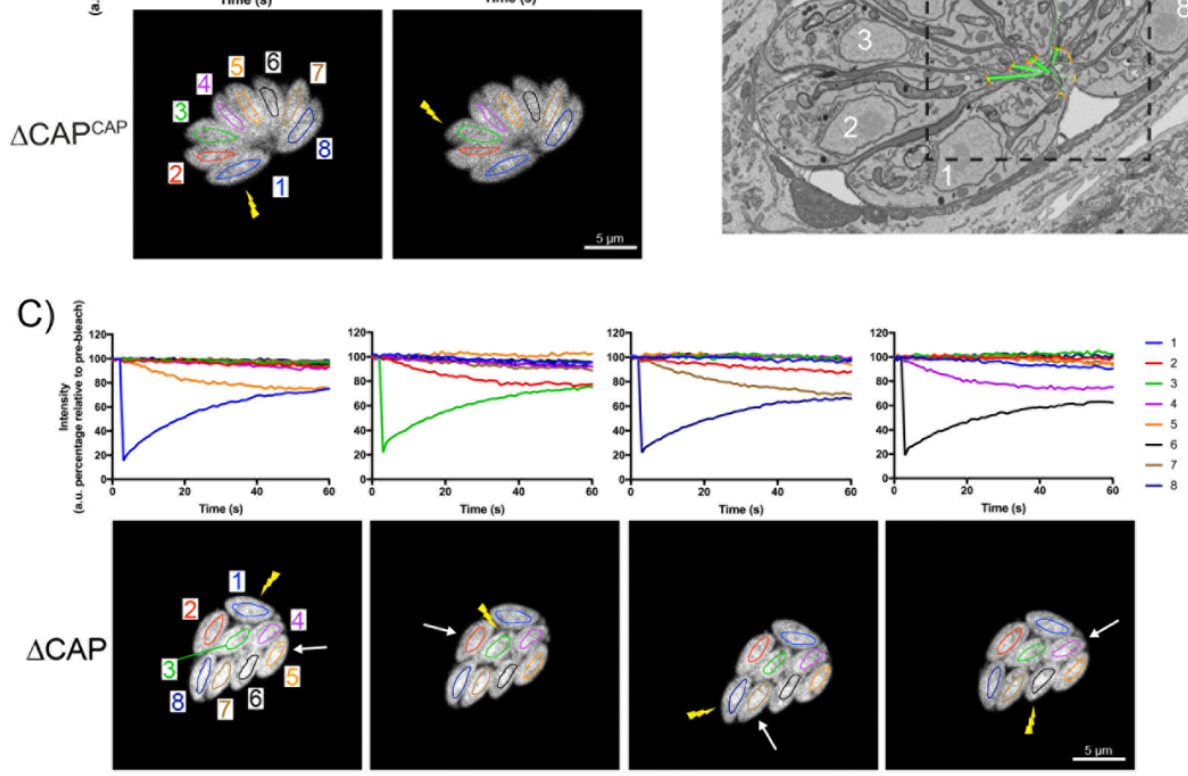

B)i)
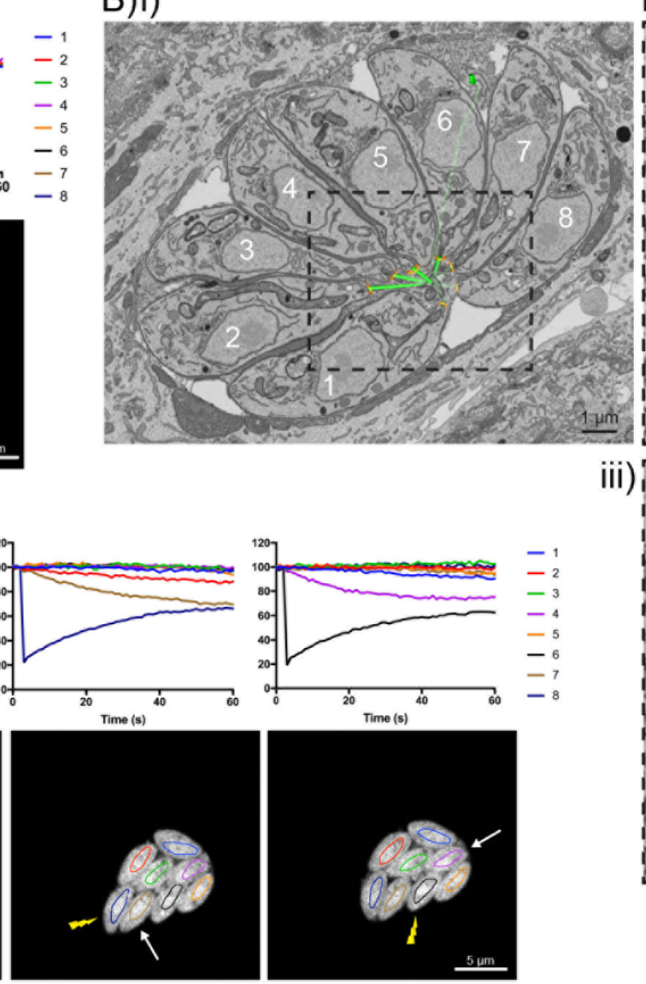

D) i)

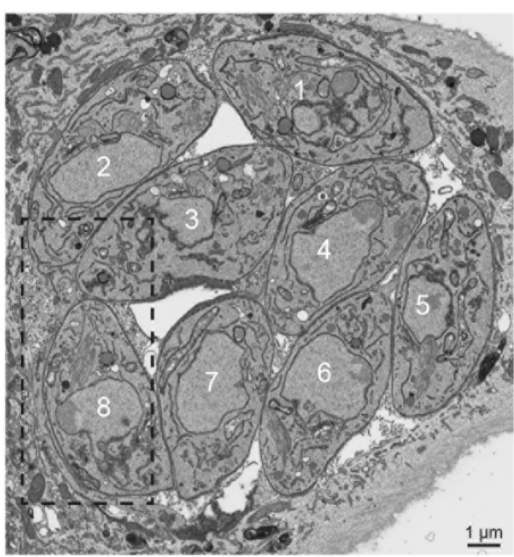

iii)

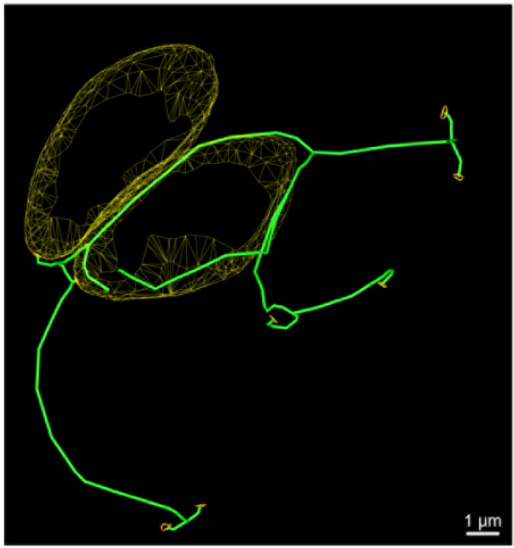

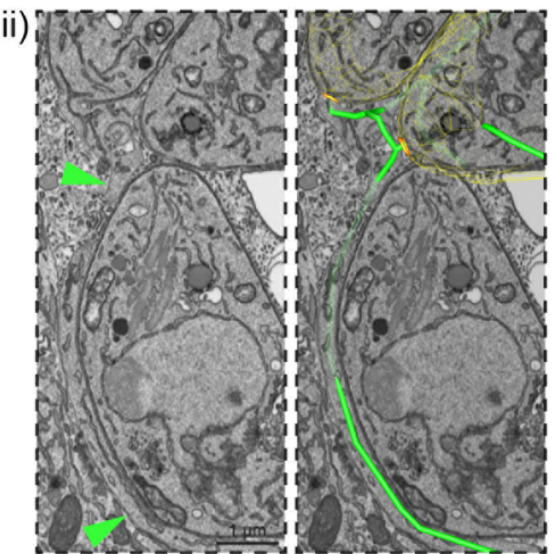

ii)

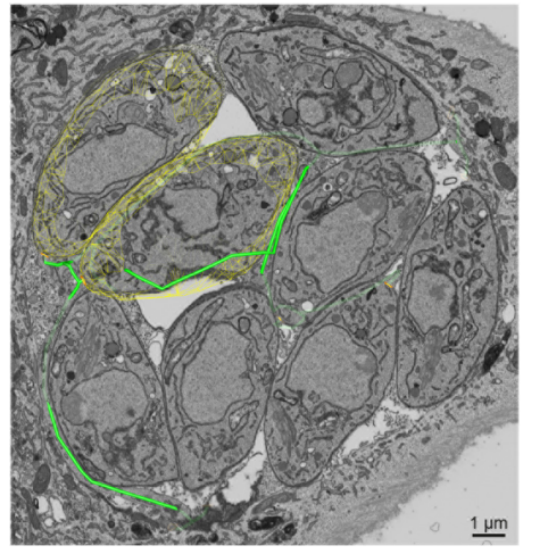

ii)

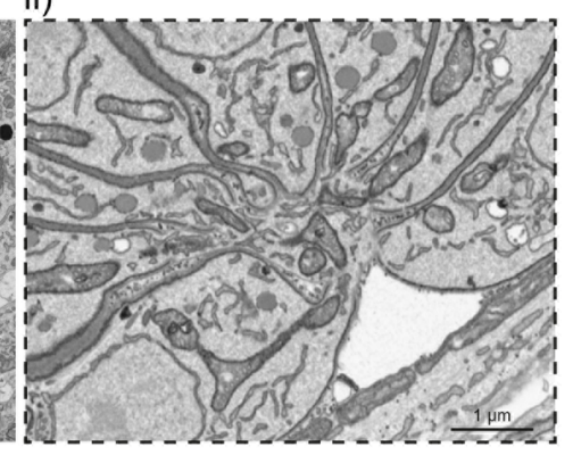

iii)

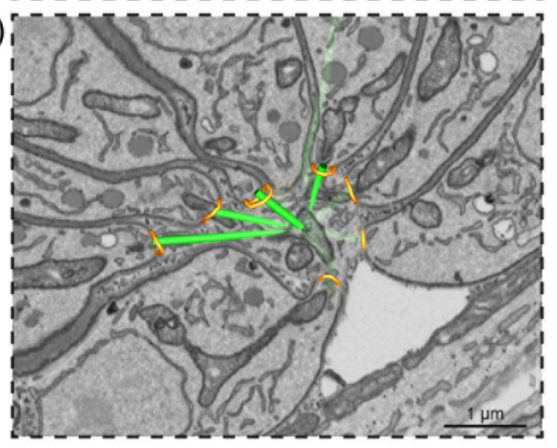


1710

1711

1712

1713

1714

1715

1716

1717

1718

1719

1720

1721

1722

1723

1724

1725

1726

1727

1728

1729

1730

1731

1732

1733

1734

1735

1736

1737

1738

1739

1740

1741

1742

1743

1744

1745

1746

1747

1748

1749

1750

1751

1752

1753

1754

1755

1756

1757

1758

1759

1760

1761

1762

1763

1764

\section{Figure 8}

A)

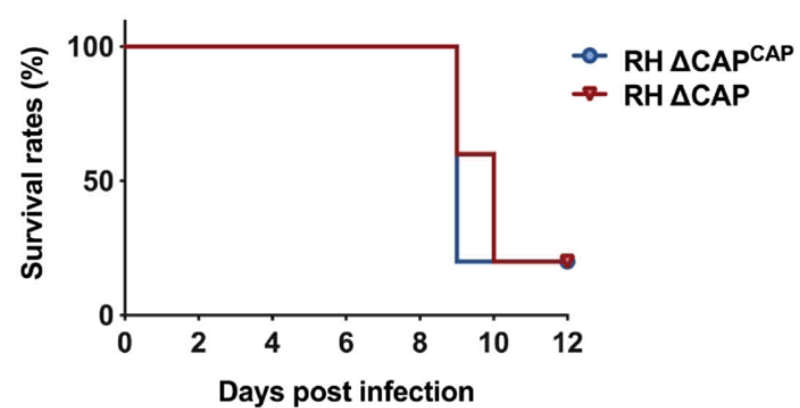

B)

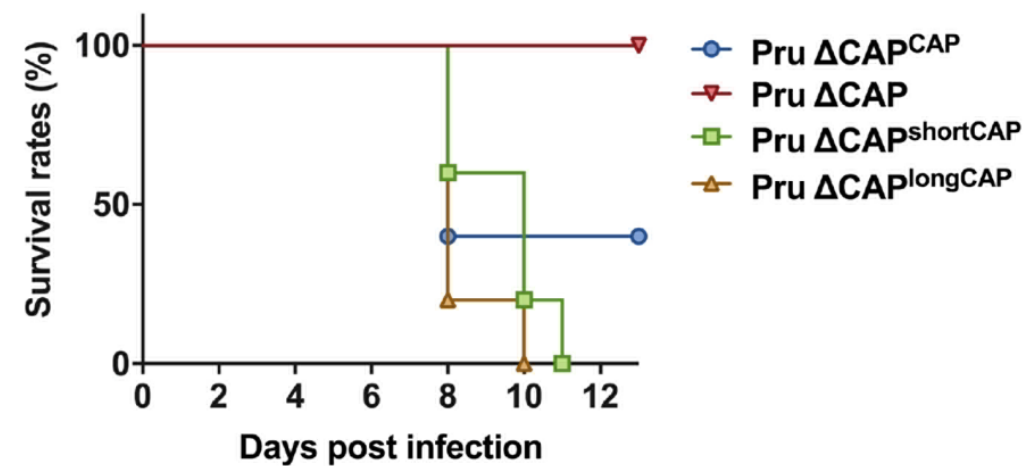

C)

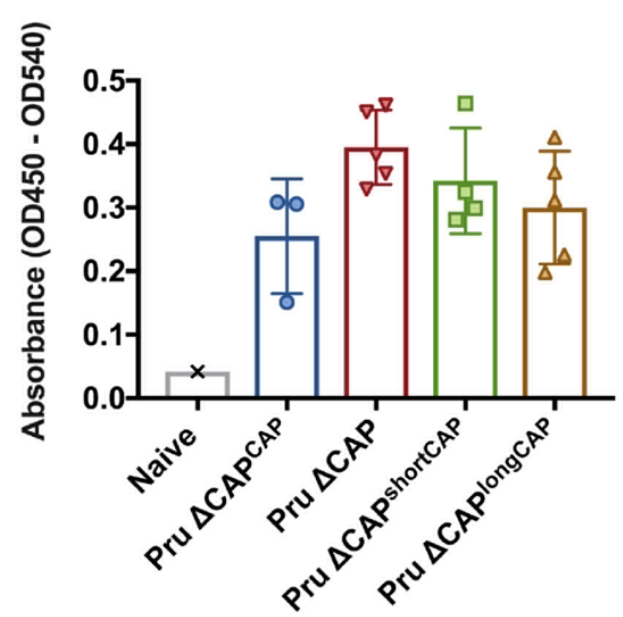

D)

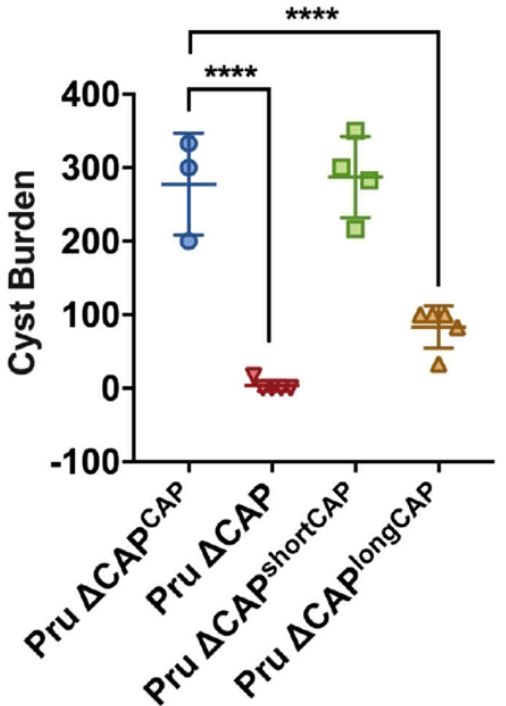


A)

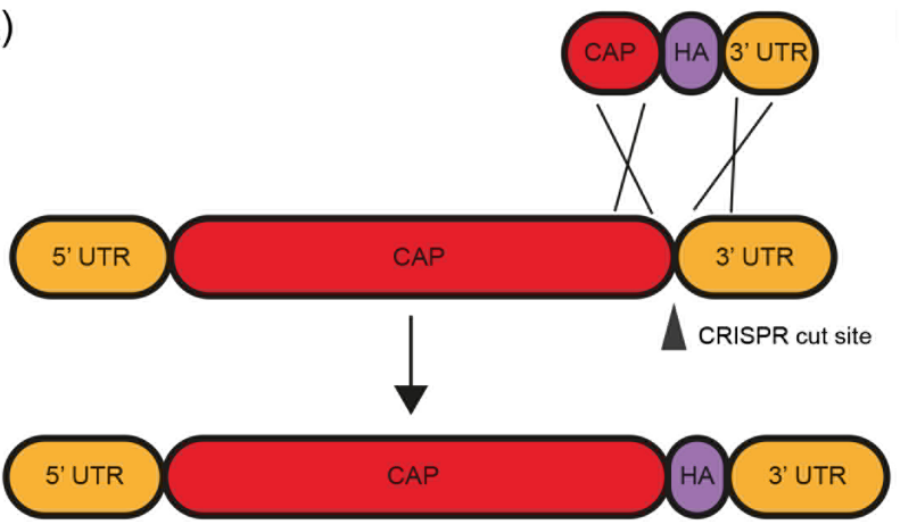

B)

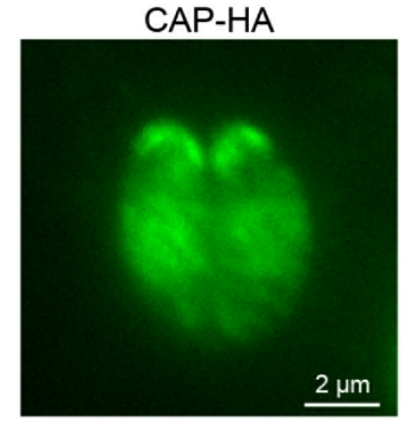




\section{Supplementary Figure 2}
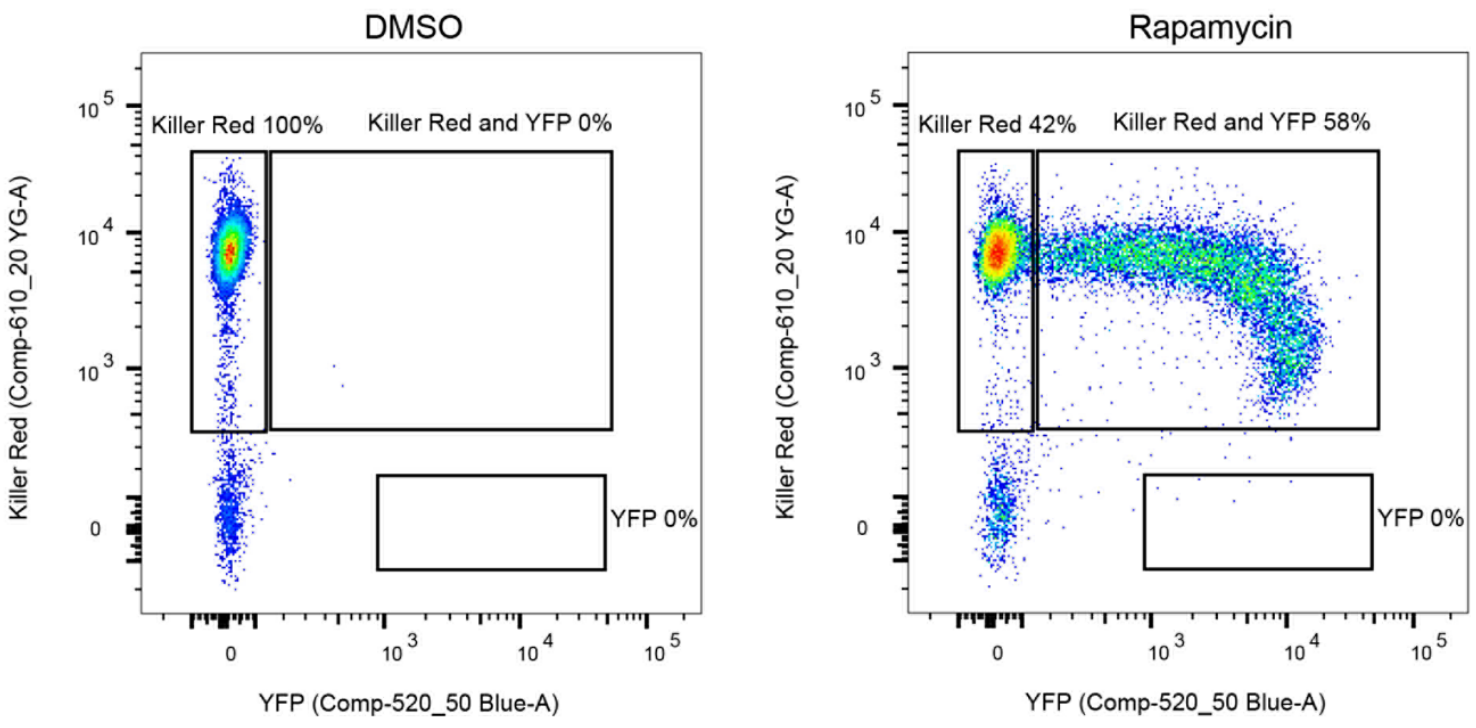
Supplementary Figure 3

1847

A) PCR3 (1237 bp, excised: 442 bp)

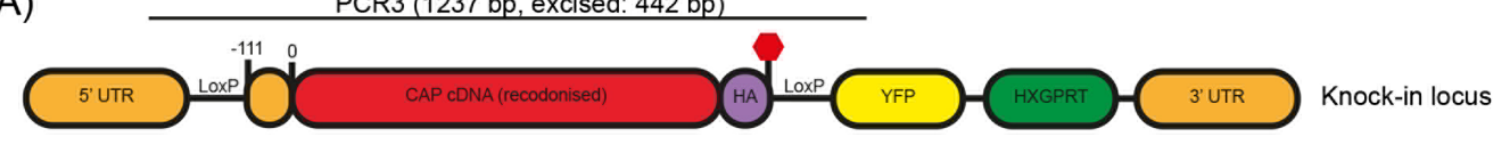



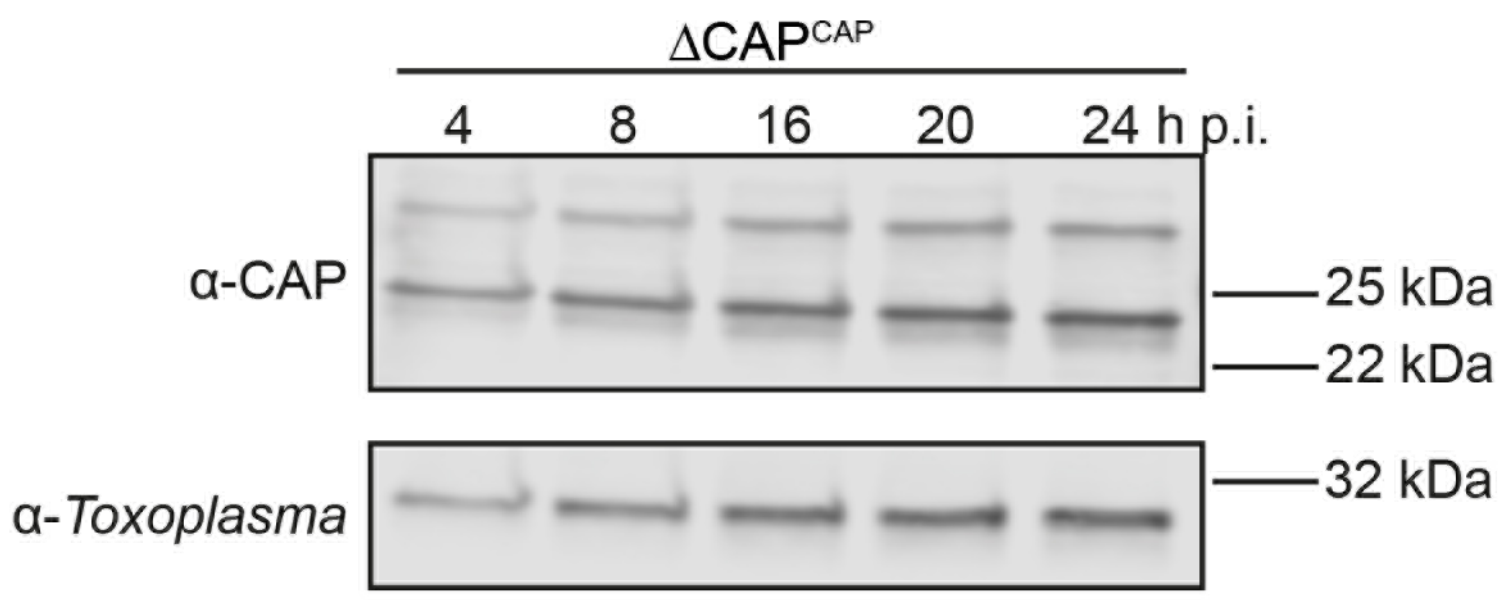

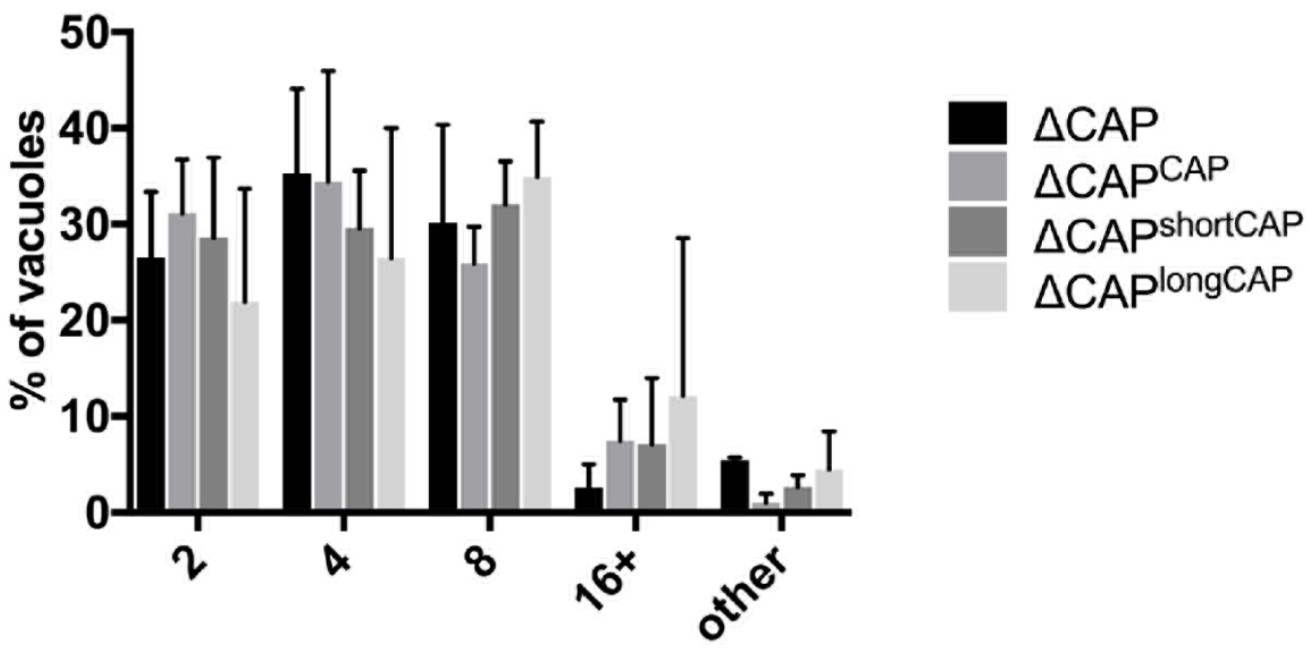
1963
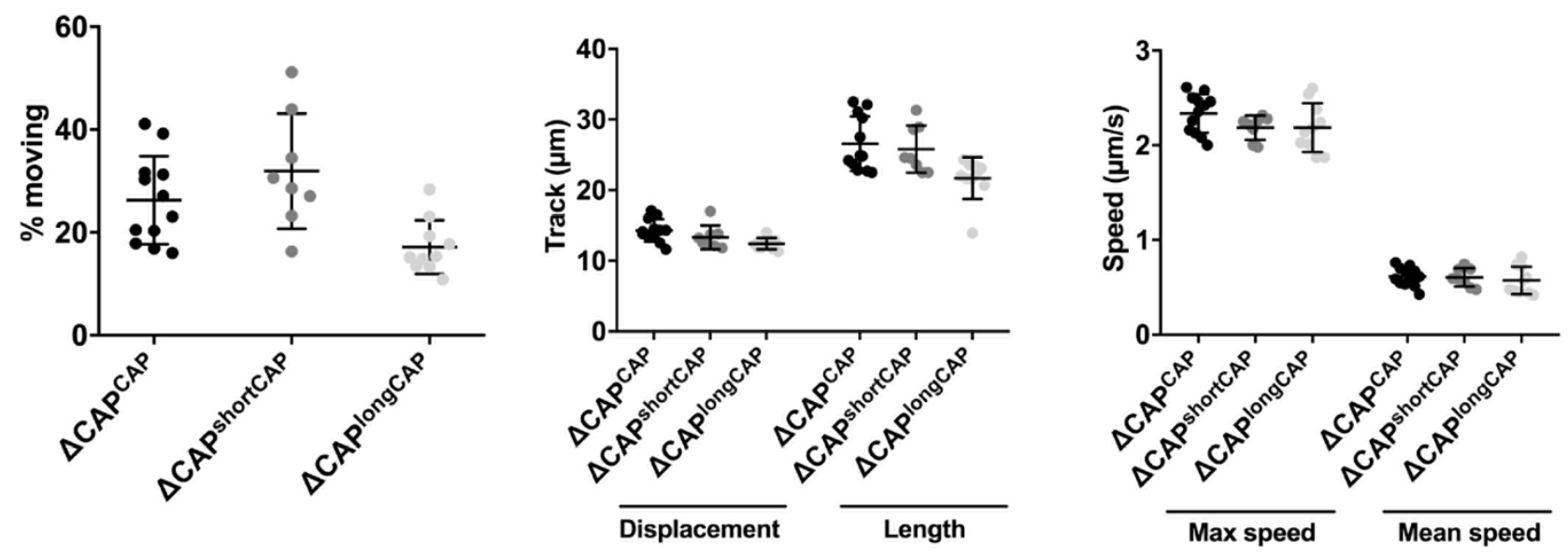
Supplementary Figure 7
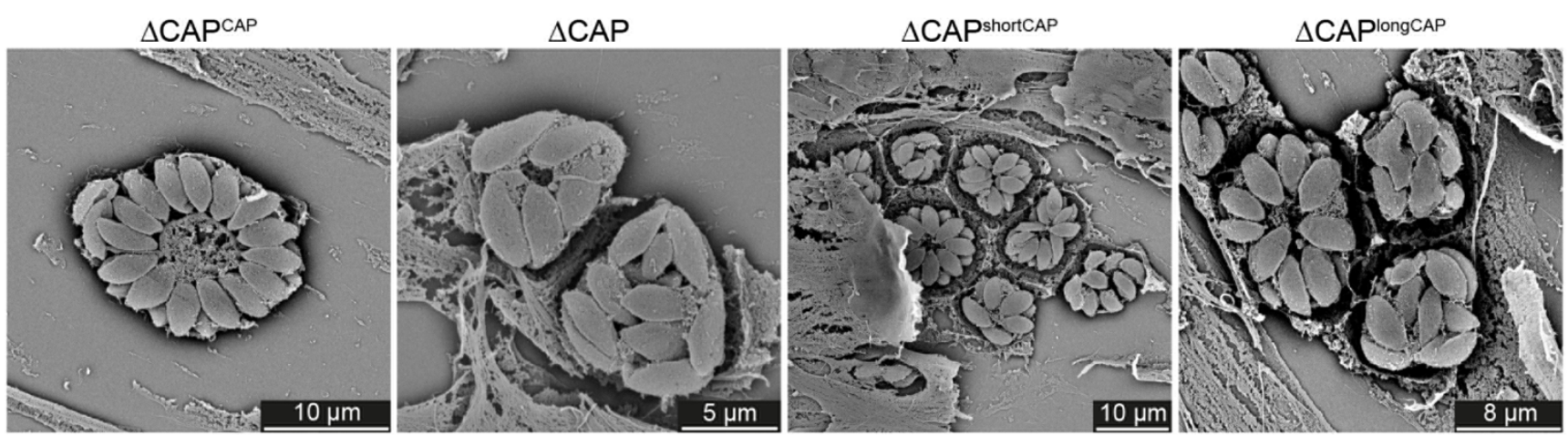

2003

2004

2005

2006

2007

2008

2009

2010

2011

2012

2013

2014

2015

2016

2017

2018

2019

2020

2021

2022

2023

2024

2025

2026

2027

2028

2029

2030

2031

2032

2033

2034

2035

2036

2037

2038

2039

2040

2041

2042

2043

2044 
Supplementary Figure 8

2046

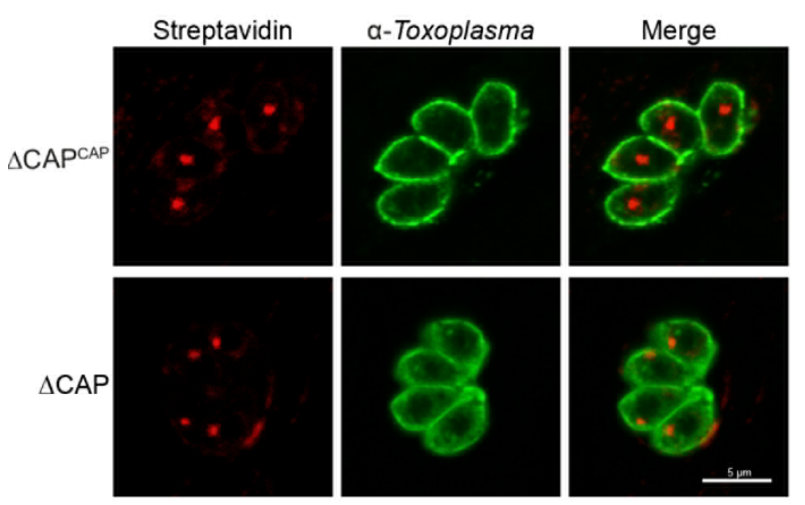




\section{Supplementary Figure 9}
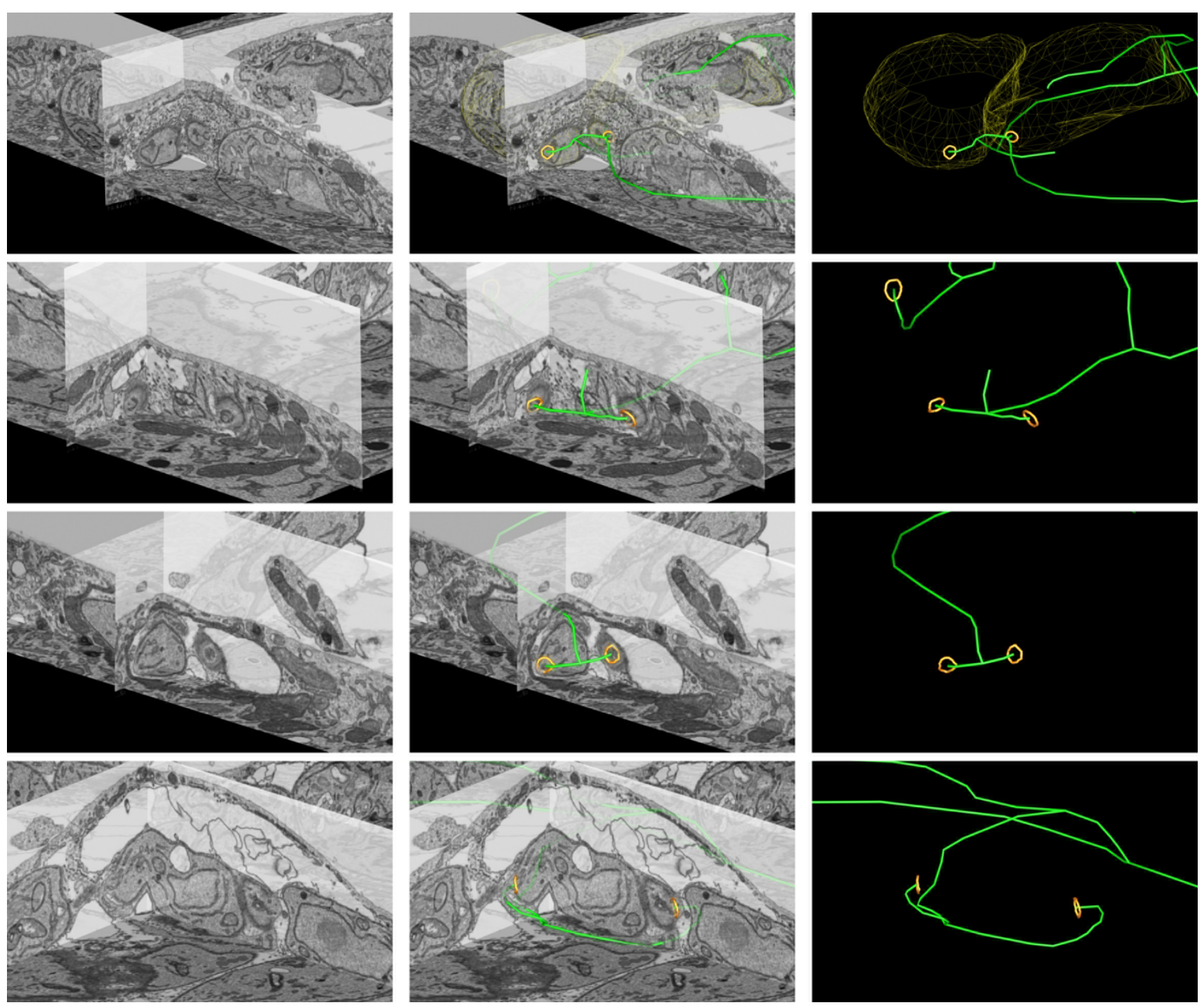